\title{
Evaluation de la stratégie de distribution à base communautaire
}

\author{
Banza Baya \\ Georges Guiella \\ Christine Ouedraogo \\ Gabriel Pictet
}

Follow this and additional works at: https://knowledgecommons.popcouncil.org/departments_sbsr-rh How does access to this work benefit you? Let us know!

\section{Recommended Citation}

Baya, Banza, Georges Guiella, Christine Ouedraogo, and Gabriel Pictet. 1998. "Evaluation de la stratégie de distribution à base communautaire," Rapport d'Evaluation. Washington, DC and Bazega: Population Council and Laboratoire de Santé Communautaire. 


\section{LABORATOIRE DE SANTE COMMUNAUTAIRE DU BAZEGA}

Direction de la santé de la famille
Unité d'enseignement et de recherche en démographie
Mwangaza Action

Population Council

\section{RAPPORT D'EVALUATION}

Evaluation de la stratégie de distribution à base communautaire.

Includes an executive summary and data fact sheets in English

Banza Baya*, Georges Guiella*, Christine Ouedraogo* et Gabriel Pictet* ${ }^{* \mu}$

\section{Décembre 1998}

* Unité d'enseignement et de recherche en démographie, Université de Ouagadougou.

${ }^{\mu}$ Département de démographie, Université de Montréal. 


\section{EXECUTIVE SUMMARY}

\section{LSC - CBD EVALUATION RESULTS AND RECOMMENDATIONS}

\section{LSC objectives}

"The long term objective of the LSC project is to assist the Ministry of Health in developing a package of community health services that will be efficient and have sustained impact on health, through culturally acceptable approaches to improving the utilization of the services, reduce the rates of fertility, morbidity and maternal and infant mortality. (...). The UERD's immediate aim is to assess the impact of the application of community based distribution of contraceptive modalities in the Bazega ${ }^{1}$."

UERD is in charge of the research and evaluation component for the LSC. It is conducting both the panel surveys (1996 and 1998) and in-depth interviews to assess changes in attitudes, knowledge and reproductive health practices.

\section{Scope of the LSC-CBD evaluation}

The LSC evaluation report concerns community-based distribution strategies in family planning. It answers the following questions ${ }^{2}$ :

1) Is the family planning intervention relevant? In other words, is it justified to implement a family planning program in the Bazèga? Are the intervention assumptions valid?

2) Is intervention implementation satisfactory? What proportion of the population was actually covered? How intensively were individuals exposed to the various components of the FP program? How was the program perceived by the general population (community), the participants (Management Committees, CBD agents, health center personnel), the target population (women of reproductive age) and the beneficiaries (users of modern contraceptives)?

3) Did the intervention have an effect (impact) on perceptions, attitudes on reproduction and fertility behavior? If statistically significant changes are observed, by which mechanisms have they been achieved?

\footnotetext{
${ }^{1}$ Research Proposal for the creation of a community health research laboratory in a rural area of Burkina Faso, submitted by UERD to the Rockefeller Foundation, March 1995. Italics ours.

${ }^{2}$ These questions are answered in detail in the complete evaluation report. This executive summary presents only the major evaluation results.
} 
4) Which intervention strategy is the most effective and sustainable? Is a community-based distribution approach more effective than center-based family planning services? Does the CBD cost recovery scheme hinder contraceptive use? Is a community-based approach more sustainable?

\section{Survey findings}

The UERD conducted surveys in 1996, 1997 and 1998 using both quantitative and qualitative methods.

- In 1996 and 1998, Total fertility rate (TFR) at respectively 6.7 and 6.4 children/woman is lower in the Bazega than in rural Burkina in 1993 at 7.3 (DHS/Burkina Faso). Infant $(<1$ yr.) and infant \& child $(<5$ yr.) mortality rates reached respectively 117 and $192 \%$ in 1996; 90 and $157192 \%$ in 1998 .

- Men and women do not discuss fertility issues with their spouses. Over $85 \%$ of the respondents never discussed the number of children they want. On average, women want three boys and two girls. Boys tend to be preferred but all respondents desire at least one girl.

- Women and men seem ignorant about the biology of human reproduction. Gender differences in knowledge on fertility matters are strong: four men out of five say they have heard about birth spacing. The proportion among women is substantially lower (three women out of five). Birth spacing is encouraged by tradition. Over $80 \%$ of both men and women say that the interval between two births should be of at least three years. This is usually achieved by post partum abstinence. The median birth interval is 37.5 months; $91.5 \%$ of the births in the last 5 years occurred more than two years after the preceding birth.

- Women say birth spacing is necessary so that mothers can rest. Men say that birth spacing is needed so that mothers can take better care of their children.

- Among married women, 4.4\% were using a modern contraceptive method in 1996 (pill: 2.3\% ; condom: 1.4\%). Contraceptive prevalence almost doubled in zone B between 1996 and 1998, from 3.6 to 8.0\% $(\mathrm{P}<0.05)$. Contraceptive prevalence also increased in zone $\mathrm{A}$, though not significantly $(\mathrm{P}=0.1)$, remained unchanged in zones C and D. Increase in contraceptive use in zones A and B is probably a result of the greater accessibility of contraceptives.

- Among women not using a contraceptive method, 41\% said in 1996 that they would use one in the future, $38 \%$ said they would not. Intentions remained unchanged after 18 months of intervention.

- The unmet need for family planning (DHS definition) decreased in zone B, from $48 \%$ in 1996 to $42 \%$ in 1998. It also decreased in zone C, remained stable in zone A (39\% versus 37\%) and increased in zone D. 
- $\quad 79 \%$ of women respondents (15-49 years) are excised. Despite the fact that it is illegal, $51 \%$ of women and $41 \%$ of men said they approved female circumcision in 1996. The proportion of respondents who say they disapprove of FGM increased significantly in all zones between 1996 and 1998.

- Induced abortion is equally illegal, yet traditional methods are available and practiced in the community.

\section{$\underline{\text { Implications and recommendations for family planning }}$}

Spouses do not communicate among themselves on fertility matters. One reason is that the need for high fertility is obvious for both. Our findings are coherent with those of other studies in rural Burkina Faso and elsewhere in rural West Africa: husbands and wives both have converging interest in high fertility ${ }^{3}$. Promotion of Family planning should therefore use gender specific messages that take into account the reasons why men and women want many children, and why they do hesitate to use modern contraceptives.

Young married women seek to space their births, rather than limit their overall fertility. They achieve this by using traditional methods (mostly post partum abstinence) It is only when women reach the age of 40 and that they have a sufficient number of sons and daughters that they may be interested in terminating their reproductive careers. Unless these age-specific fertility intentions change, a substantial reduction fertility rate is unlikely.

Current ignorance on human reproduction is not compatible with the distribution of modern contraceptives. The CBD agents and health center personnel need to improve people's knowledge on the biology of human reproduction so that women and men may use more efficiently natural family planning methods, understand the advantages of modern methods and use them correctly. Many women believe that the pill can induce abortions and that it causes sterility.

The obstacles to contraceptive use are great. Both the community and a significant portion of men are wary of modern contraception and of giving women the possibility of controlling their fertility. Hence, confidentiality is crucial. Women need to be convinced of the discretion of the health personnel in the local health centers and of the CBD in zone B.

\footnotetext{
${ }^{3}$ Poirier, Guiella, Fondements socio-économiques de la fécondité chez les Mossi du plateau Central, Burkina Faso, Travaux de l’UERD n 1 , Ouagadougou, mai 1996.
} 


\section{$\underline{\text { Evaluation results and recommendations for CBD strategy }}$}

People do not feel that family planning is the most urgent priority for their community. In fact, some CBD are stigmatized for encouraging women to indulge in pre-marital or extra-marital sex. To encourage and sustain community efforts in zone $\mathrm{B}$, it is necessary to implement activities addressing health problems perceived by the community as being important or urgent. Indeed, the most popular aspect of the CBD intervention is the sale of chloroquine and aspirin.

Activities against FGM should be lead independently and by different people. These activities require a different approach, specific skills and training.

Community participation in zone B is not perceptible. Neither the Management Committees nor the survey respondents feel concerned by CBD activities.

At all levels, motivation is inadequate. CBD agents are not regularly supervised. They do not fully understand the activity report forms they are supposed to fill in. Profits from the sale of contraceptives are too low to be considered as sufficient incentive.

CBD coverage is low. According to survey data, on average, each CBD agent visited less than 21 women in 18 months (barely one a month!). Only $17 \%$ of the women remembered having been visited at least once by a CBD agent.

The CBD strategy was relatively successful in increasing the accessibility of modern contraceptives (mostly the pill). As a result, contraceptive use increased in Zone B while intentions to use contraceptives remained stable. This suggests that the CBD agents supplied women who were already convinced of the advantages of contraceptives. The CBD agents probably had little effect on perceptions.

Young women ( $<25$ yrs.) were not visited as frequently as older women. This is a shame, not least because young unmarried women are the most susceptible to terminate unwanted pregnancies. Specific activities should be implemented for adolescents.

CBD agents need more training. Training is in itself an incentive, and it is a requirement if the intervention's strategy is to improve perceptions on modern contraception.

More CBD need to be recruited and trained. There are two CBD per village, independently of the village's size or population. As a result, many CBD agents cannot cover more than a small fraction of their community.

CBD agents should be recruited for a limited period (say 18 months). In the present system, CBD agents do not feel they need to work to keep their job (i.e. the CBD bicycle). 
MoH involvement in CBD management is inadequate. CBD strategies require large amounts of organizing, training, supervision. It requires the capacity to listen to people's needs and to react quickly and efficiently. The $\mathrm{MoH}$ has not yet demonstrated it can meet, let alone sustain, these requirements in the Bazega.

In its current form, the CBD strategy is neither efficient nor sustainable. It needs to be greatly improved before replication or upscaling can be envisaged. 


\begin{tabular}{|c|c|c|c|c|c|c|c|c|c|c|c|c|c|c|c|}
\hline \multirow{3}{*}{\begin{tabular}{|l} 
\\
Individual characteristics $(\%) *$
\end{tabular}} & \multicolumn{3}{|c|}{ A } & \multicolumn{3}{|c|}{ B } & \multicolumn{3}{|c|}{$\mathrm{C}$} & \multicolumn{3}{|c|}{$\mathrm{D}$} & \multicolumn{3}{|c|}{ All } \\
\hline & 1998 & 1996 & $\%$ var & 1998 & 1996 & $\%$ var & 1998 & 1996 & $\%$ var & 1998 & 1996 & $\%$ var & 1998 & 1996 & $\%$ var \\
\hline & & & & & & & & & & & & & & & \\
\hline Women with no schooling & & 90.8 & - & & 91.3 & & & 92.3 & - & & 95.6 & & & 92.4 & \\
\hline Men with no schooling & & 93.3 & & & 90.6 & & & 91.8 & & & 91.1 & & & 91.7 & \\
\hline Illiterate women & & 95.1 & & & 92.3 & & & 94.2 & & & 95.8 & & & 94.3 & \\
\hline Illiterate men & & 90.4 & - & & 82.9 & & & 88.8 & - & & 86.1 & & & 87.1 & \\
\hline Religion & & & & & & & & & & & & & & & \\
\hline - Animist & & 10.0 & - & & 12.6 & & & 12.6 & - & & 33.6 & & & 16.7 & \\
\hline - Catholic (DHS: Christian) & & 40.3 & & & 57.6 & & & 18.3 & & & 42.5 & & & 38.8 & \\
\hline - Protestant & & 5.5 & & & 7.6 & & & 4.4 & & & 6.6 & & & 5.9 & \\
\hline - Muslim & & 43.9 & 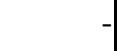 & & 22.2 & & & 64.4 & - & & 17.2 & & & 38.3 & \\
\hline Mossi & & 98.1 & - & & 96.6 & & & 96.1 & - & & 98.4 & & & 97.3 & \\
\hline Health indicators & & & & & & & & & & & & & & & \\
\hline Infant mortality & 80 & 114 & $-29.9 \%$ & 80 & 109 & $-26.5 \%$ & 134 & 140 & $-4.2 \%$ & 55 & 94 & $-42.0 \%$ & 90.0 & 116.9 & $-23.0 \%$ \\
\hline Infant and child mortality ( $0-4$ yrs $)$ & 159 & 178 & $-10.7 \%$ & 129 & 151 & $-14.8 \%$ & 198 & 209 & $-5.5 \%$ & 129 & 225 & $-42.6 \%$ & 157.2 & 192.0 & $-18.1 \%$ \\
\hline Prevalence of excision & & & & & & & & & & & & & & & \\
\hline all women & & 95.2 & & & 83.3 & & & 84.8 & & & 96.4 & & & 89.6 & \\
\hline $15-24$ yrs & & 94.1 & - & & 79.3 & & & 78.5 & - & & 96.3 & & & 86.8 & \\
\hline $35-49$ yrs & & 96.0 & & & 85.6 & & & 86.5 & & & 99.0 & & & 91.4 & \\
\hline Number of prenatal visits (latest bi & & & & & & & & & & & & & & & \\
\hline 0 & 4.4 & 6.3 & $-30.5 \%$ & 5.3 & 6.3 & $-14.7 \%$ & 13.2 & 18.6 & $-29.0 \%$ & 7.4 & 7.2 & $2.4 \%$ & 8.0 & 10.3 & $-22.4 \%$ \\
\hline 1 & 5.3 & 8.6 & $-38.5 \%$ & 3.1 & 8.5 & $-63.5 \%$ & 5.8 & 5.7 & $1.0 \%$ & 6.4 & 8.8 & $-26.9 \%$ & 5.2 & 7.7 & $-33.3 \%$ \\
\hline $2-3$ & 53.7 & 57.0 & $-5.7 \%$ & 44.4 & 47.2 & $-5.8 \%$ & 42.4 & 36.1 & $17.3 \%$ & 52.2 & 54.6 & $-4.4 \%$ & 47.7 & 48.0 & $-0.7 \%$ \\
\hline 4 or more & 35.7 & 27.6 & $29.3 \%$ & 43.6 & 36.9 & $17.9 \%$ & 33.6 & 36.9 & $-9.0 \%$ & 34.0 & 29.4 & $15.7 \%$ & 36.5 & 32.8 & $11.4 \%$ \\
\hline Does not know / Not available & 0.9 & 0.5 & $94.7 \%$ & 3.6 & 1.1 & $212.9 \%$ & 5.1 & 2.7 & $91.0 \%$ & 0.0 & 0.0 & & 2.6 & 1.2 & $124.7 \%$ \\
\hline
\end{tabular}




\begin{tabular}{|c|c|c|c|c|c|c|c|c|c|c|c|c|c|c|c|}
\hline & \multicolumn{3}{|c|}{ A } & \multicolumn{3}{|c|}{$\mathrm{B}$} & \multicolumn{3}{|c|}{$\mathrm{C}$} & \multicolumn{3}{|c|}{$\mathrm{D}$} & \multicolumn{3}{|c|}{ All } \\
\hline & 1998 & 1996 & $\%$ var & 1998 & 1996 & $\%$ var & 1998 & 1996 & $\%$ var & 1998 & 1996 & $\%$ var & 1998 & 1996 & $\%$ van \\
\hline \multicolumn{16}{|l|}{ Number of tetanos injections } \\
\hline 0 & 30.6 & 28.2 & $8.5 \%$ & 42.3 & 36.0 & $17.5 \%$ & 34.6 & 46.7 & $-25.9 \%$ & 52.6 & 45.3 & $16.1 \%$ & 39.3 & 39.0 & $0.6 \%$ \\
\hline 1 & 18.9 & 27.8 & $-31.8 \%$ & 20.7 & 28.7 & $-27.9 \%$ & 20.8 & 22.0 & $-5.1 \%$ & 17.3 & 19.6 & $-11.3 \%$ & 19.6 & 24.4 & $-19.8 \%$ \\
\hline 2 or more & 48.6 & 43.1 & $13.0 \%$ & 35.2 & 33.5 & $5.0 \%$ & 42.4 & 31.3 & $35.4 \%$ & 29.1 & 35.2 & $-17.4 \%$ & 39.4 & 35.9 & $9.7 \%$ \\
\hline Does not know / Not available & 1.8 & 1.0 & $88.3 \%$ & 1.9 & 1.8 & $2.7 \%$ & 2.1 & 0.0 & & 1.0 & 0.0 & & 1.8 & 0.7 & $168.2 \%$ \\
\hline Babies breast fed "first milk" & & & & & & & & & & & & & & & \\
\hline (last child born 18 months before surv.) & 51.5 & 42.7 & $20.6 \%$ & 62.8 & 65.3 & $-3.8 \%$ & 56.3 & 39.8 & $41.4 \%$ & 71.0 & 88.7 & $-19.9 \%$ & 59.8 & 57.0 & $4.9 \%$ \\
\hline Prevalence of diarrhea & 8.1 & 16.9 & $-52.0 \%$ & 9.0 & 24.9 & $-64.0 \%$ & 12.0 & 20.8 & $-42.4 \%$ & 7.2 & 25.0 & $-71.3 \%$ & 9.3 & 21.8 & $-57.3 \%$ \\
\hline Prevalence of malaria & 2.2 & 16.5 & $-86.5 \%$ & 7.2 & 22.9 & $-68.7 \%$ & 6.5 & 14.0 & $-53.2 \%$ & 4.8 & 13.8 & $-65.0 \%$ & 5.2 & 16.6 & $-68.5 \%$ \\
\hline$\%$ Women who disaproves of FGM & 68.6 & 33.9 & $102.6 \%$ & 65.9 & 43.9 & $50.1 \%$ & 62.1 & 50.3 & $23.5 \%$ & 61.8 & 38.3 & $61.3 \%$ & 64.6 & 42.0 & $53.9 \%$ \\
\hline \multicolumn{16}{|l|}{ Fertility indicators } \\
\hline Birth rate & 33 & 40 & $-17.2 \%$ & 31 & 35 & $-12.5 \%$ & 32 & 41 & $-21.5 \%$ & 64 & 69 & $-7.6 \%$ & 32.0 & 37.9 & $-15.5 \%$ \\
\hline GFR & 208 & 217 & $-4.1 \%$ & 174 & 189 & $-7.9 \%$ & 185 & 229 & $-19.1 \%$ & 211 & 186 & $13.6 \%$ & 193.1 & 206.5 & $-6.5 \%$ \\
\hline TFR & 6.6 & 6.8 & $-2.9 \%$ & 6.1 & 6.3 & $-3.6 \%$ & 6.1 & 7.6 & $-19.5 \%$ & 6.9 & 6.1 & $14.7 \%$ & 6.4 & 6.7 & $-4.6 \%$ \\
\hline \multicolumn{16}{|l|}{ Women: mean number of } \\
\hline live births (40-49 yrs) & & 7 & & & 7 & & & 7 & & & 7 & & 7.3 & 7.3 & $0.0 \%$ \\
\hline live births (45-49 yrs) & & 7 & & & 8 & & & 7 & & & 7 & & 7.4 & 7.4 & $0.0 \%$ \\
\hline surviving children (45-49 yrs) & & 5 & & & 6 & & & 5 & & & 5 & & 5.3 & 5.3 & $0.0 \%$ \\
\hline \multicolumn{16}{|l|}{ Men: mean number of } \\
\hline live births $(>50$ yrs $)$ & 11.6 & 11.6 & $0.0 \%$ & 12.6 & 12.6 & $0.0 \%$ & 11.3 & 11.3 & $0.0 \%$ & 11.0 & 11.0 & $0.0 \%$ & 11.6 & 11.6 & $0.0 \%$ \\
\hline live births (>60 yrs) & 12.8 & 12.8 & $0.0 \%$ & 12.3 & 12.3 & $0.0 \%$ & 11.2 & 11.2 & $0.0 \%$ & 12.3 & 12.3 & $0.0 \%$ & 12.1 & 12.1 & $0.0 \%$ \\
\hline surviving children (>60 yrs) & 8.4 & 8.4 & $0.0 \%$ & 9.5 & 9.5 & $0.0 \%$ & 8.0 & 8.0 & $0.0 \%$ & 8.3 & 8.3 & $0.0 \%$ & 8.6 & 8.6 & $0.0 \%$ \\
\hline \multicolumn{16}{|l|}{ Fertility determinants } \\
\hline median age at first marriage (women)* & & 17 & & & 17 & & & 17 & & & 17 & & 17.0 & 17.0 & $0.0 \%$ \\
\hline$\%$ belonging to a polygamous union & 60.3 & 50.4 & $19.8 \%$ & 55.5 & 41.7 & $33.3 \%$ & 62.6 & 55.4 & $13.0 \%$ & 57.0 & 47.3 & $20.4 \%$ & 59.1 & 49.0 & $20.7 \%$ \\
\hline median birth interval & 37.5 & 37.5 & $0.0 \%$ & 36.5 & 36.5 & $0.0 \%$ & 37.6 & 37.6 & $0.0 \%$ & 36.5 & 36.5 & $0.0 \%$ & 37.5 & 37.5 & $0.0 \%$ \\
\hline
\end{tabular}




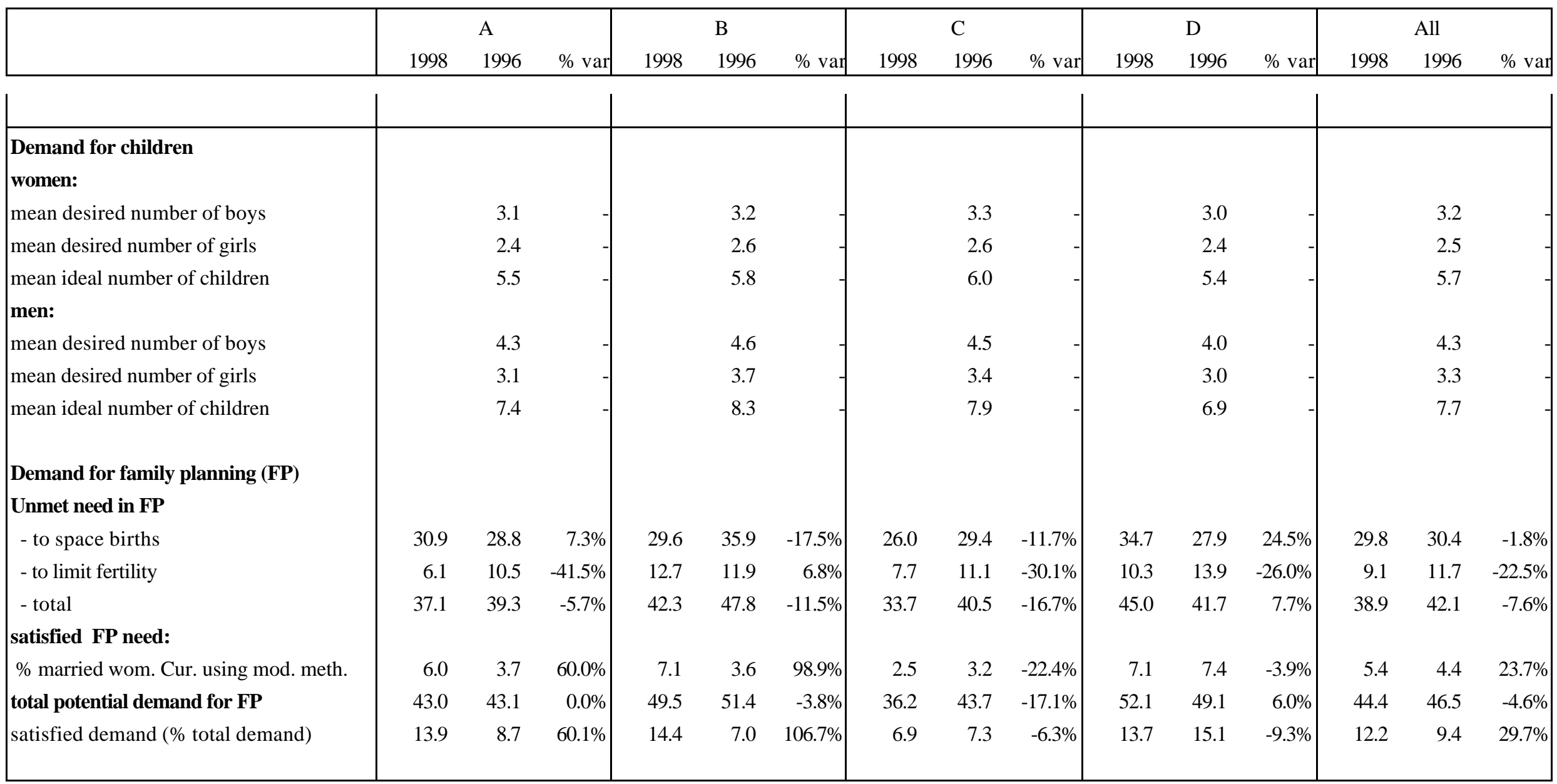




\begin{tabular}{|c|c|c|c|c|c|c|c|c|c|c|c|c|c|c|c|}
\hline & \multicolumn{3}{|c|}{ A } & \multicolumn{3}{|c|}{$\mathrm{B}$} & \multicolumn{3}{|c|}{$\mathrm{C}$} & \multicolumn{3}{|c|}{$\mathrm{D}$} & \multicolumn{3}{|c|}{ All } \\
\hline & 1998 & 1996 & $\%$ var & 1998 & 1996 & $\%$ van & 1998 & 1996 & $\%$ var & 1998 & 1996 & $\%$ var & 1998 & 1996 & $\%$ var \\
\hline \multicolumn{16}{|c|}{ KAP on contraception } \\
\hline$\%$ married women & & & & & & & & & & & & & & & \\
\hline -a modern method & 58.4 & 45.6 & $28.1 \%$ & 65.2 & 57.7 & $13.0 \%$ & 47.9 & 53.9 & $-11.0 \%$ & 68.8 & 65.8 & $4.5 \%$ & 59.0 & 55.3 & $6.5 \%$ \\
\hline -the pill & 35.3 & 32.7 & $7.8 \%$ & 30.7 & 29.3 & $4.6 \%$ & 29.7 & 30.0 & $-1.0 \%$ & 31.8 & 13.5 & $136.0 \%$ & 31.7 & 26.8 & $18.3 \%$ \\
\hline -IUD & 18.1 & 10.8 & $66.7 \%$ & 28.7 & 27.0 & $6.3 \%$ & 19.1 & 21.5 & $-11.5 \%$ & 28.5 & 26.9 & $5.6 \%$ & 23.1 & 21.3 & $8.6 \%$ \\
\hline -injections & 36.5 & 28.8 & $26.8 \%$ & 38.2 & 36.1 & $5.7 \%$ & 27.1 & 39.3 & $-31.1 \%$ & 45.8 & 42.9 & $6.8 \%$ & 35.9 & 36.7 & $-2.3 \%$ \\
\hline -condoms & 58.4 & 44.4 & $31.5 \%$ & 56.1 & 51.2 & $9.5 \%$ & 42.3 & 44.4 & $-4.8 \%$ & 58.4 & 43.1 & $35.5 \%$ & 52.8 & 45.6 & $15.7 \%$ \\
\hline \multicolumn{16}{|c|}{$\%$ married women currently using: } \\
\hline a modern method & 5.9 & 3.7 & $60.0 \%$ & 8.0 & 3.6 & $122.2 \%$ & 2.7 & 3.2 & $-15.6 \%$ & 7.4 & 7.3 & $-1.4 \%$ & 5.4 & 4.4 & $23.7 \%$ \\
\hline -the pill & 2.5 & 1.9 & $35.8 \%$ & 4.9 & 1.5 & $222.6 \%$ & 0.8 & 1.7 & $-52.6 \%$ & 3.6 & 4.2 & $-14.8 \%$ & 2.8 & 2.3 & $22.9 \%$ \\
\hline -IUD & 0.5 & 0.8 & $-36.0 \%$ & 0.3 & 0.4 & $-7.8 \%$ & 0.3 & 0.1 & $89.8 \%$ & 0.2 & 0.4 & $-44.9 \%$ & 0.3 & 0.4 & $-19.9 \%$ \\
\hline -injections & 0.9 & 0.2 & $433.5 \%$ & 0.9 & 0.2 & $360.8 \%$ & 0.4 & 0.1 & $184.7 \%$ & 1.0 & 0.2 & $451.3 \%$ & 0.8 & 0.2 & $350.8 \%$ \\
\hline -condoms & 1.6 & 0.7 & $140.1 \%$ & 1.0 & 1.5 & $-30.9 \%$ & 0.8 & 0.9 & $-5.1 \%$ & 1.7 & 2.7 & $-37.0 \%$ & 1.2 & 1.4 & $-9.2 \%$ \\
\hline traditional method & 5.1 & 12.0 & $-57.9 \%$ & 8.7 & 21.7 & $-59.9 \%$ & 7.7 & 18.2 & $-57.5 \%$ & 6.3 & 29.6 & $-78.8 \%$ & 7.0 & 20.0 & $-64.8 \%$ \\
\hline any method & 11.0 & 15.8 & $-30.0 \%$ & 15.9 & 25.3 & $-37.4 \%$ & 10.2 & 21.4 & $-52.2 \%$ & 13.4 & 37.0 & $-63.8 \%$ & 12.5 & 24.4 & $-48.9 \%$ \\
\hline
\end{tabular}




\section{LABORATOIRE DE SANTE COMMUNAUTAIRE DU BAZEGA}

\section{EVALUATION DBC : PRINCIPAUX RESULTATS ET RECOMMANDATIONS}

\section{Principaux résultats.}

L'UERD a mené des enquêtes quantitatives en 1996 et 1998, et des enquêtes qualitatives en 1997 et 1998.

- En 1996 et en 1998, l'indice synthétique de fécondité était respectivement de 6,7 et 6,4 enfants par femme, ce qui est plus faible que la moyenne rurale de 7.3 en 1993 (EDS/Burkina Faso). Les taux de mortalité infantile $(<1$ an) et infanto-juvénile ( $<5$ ans) ont atteint respectivement 117 et $192 \%$ en $1996 ; 90$ et $157 \%$ en 1998 .

- Les hommes et les femmes ne discutent pas entre eux de leurs désirs de fécondité. Plus de $85 \%$ des femmes mariées n'ont jamais discuté avec leur conjoint du nombre d'enfants qu'elles désirent. En moyenne, les femmes désirent trois garçons et deux filles. Si les garçons sont préférés, toutes les femmes souhaitent avoir au moins une fille.

- Les connaissances des femmes comme des hommes en matière de reproduction sont limitées, particulièrement chez les jeunes filles. Les différences entre sexe des connaissances concernant la fécondité sont importantes : Quatre hommes sur cinq disent qu'ils ont entendu parler de méthodes d'espacement des naissances. La proportion chez les femmes est plus faible (trois femmes sur cinq).

- L'espacement des naissances est encouragé par la tradition. Plus de $80 \%$ des hommes et des femmes disent qu'il faut espacer les naissances d'au moins trois ans. La durée médiane de l'intervalle intergénésique est de 37,5 mois ; $91.5 \%$ des naissances des 5 dernières années ont eu lieu plus de 2 ans après la naissance précédente.

- Les femmes disent en premier qu'il est important d'espacer les naissances pour qu'elles puissent se reposer. Les hommes disent en premier que c'est pour permettre aux mères de mieux s'occuper des enfants.

- Parmi les femmes mariées, 4,4\% utilisaient une méthode moderne en 1996 (pilule : 2,3\% ; condom : 1,4\%). La prévalence contraceptive a doublé dans la zone B entre 1996 et 1998, de 3,6 à 8,0\% 
$(\mathrm{P}<0.05)$. Elle a aussi augmenté, mais de façon moins substantielle, dans la zone $\mathrm{A}(\mathrm{P}=0,1)$ et est demeurée stable dans les zones $\mathrm{C}$ et $\mathrm{D}$ (variations non significatives). L'accroissement de la prévalence contraceptive dans les zones B est probablement due à la plus grande accessibilité des contraceptifs.

- Parmi les femmes qui n'utilisaient pas de méthode contraceptive moderne, $41 \%$ ont déclaré en 1996 qu'elles en utiliseraient à l'avenir, 38\% qu'elles n'en utiliseraient jamais. Le niveau des intentions est resté stable après 18 mois d'intervention.

- Les besoins non satisfaits de PF(selon la définition de l'EDS) ont diminué en Zone B, de 48\% en 1996 à $42 \%$ en 1998. Ils ont aussi décru en Zone C, et sont restés stables en Zone A (39\% contre 37\% ) et ont cru en Zone D.

- $\quad 79 \%$ des femmes (15-49 ans) sont excisées. En 1996, malgré son illégalité, $51 \%$ des femmes et $41 \%$ des hommes ont déclaré approuver la pratique de l'excision. Le niveau de désapprobation de l'excision a fortement augmenté entre 1996 et1998, et ce dans toutes les zones.

- L'avortement traditionnel est pratiqué dans les quatre zones. Près d'une femme sur quatre connaît dans son entourage proche une femme qui a avorté.

\section{Implications et recommandations pour la planification familiale.}

Les époux ne parlent pas entre eux sur les questions de PF. Une raison à cela est que le besoin d'avoir "beaucoup" d'enfants est évident pour les deux, mais probablement pour des raisons différentes". La promotion de la PF doit donc utiliser des messages spécifiques pour chaque sexe et doit tenir compte des réticences des uns et des autres à utiliser des méthodes modernes de contraception.

\section{Les jeunes femmes mariées cherchent plus à espacer leurs naissances qu'à limiter leur}

fécondité. Elles atteignent cet objectif principalement par la pratique de l'abstinence post partum. Ce n'est que lorsqu'une femme atteint un certain âge (environ 40 ans) ou un certain nombre de fils et de filles qu'elle choisira d'arrêter d'avoir des enfants. Tant que ces intentions ne changent pas, il est peu probable qu'une plus grande accessibilité de contraceptifs ait un impact substantiel sur la fécondité.

\footnotetext{
${ }^{4}$ Poirier, Guiella, Fondements socio-économiques de la fécondité chez les Mossi du plateau Central, Burkina Faso, Travaux de l’UERD n²1, Ouagadougou, mai 1996.
} 


\section{L'état actuel des connaissances des bénéficiaires concernant la reproduction humaine n'est pas} compatible avec la distribution de contraceptifs. Les agents DBC et le personnel des FS doivent améliorer les connaissances de la population sur la biologie de la procréation pour que ceux-ci utilisent plus volontiers, et de façon plus efficace, des méthodes contraceptives, qu'elles soient "artificielles" ou "naturelles". Par exemple, beaucoup de femmes prennent la pilule dans l'intention d'avorter.

Les obstacles culturels à l'utilisation contraceptive sont très importants. Beaucoup d'hommes et de femmes craignent qu'en permettant aux femmes de contrôler leur fécondité elles soient tentées de mener “des vies dissolues". La confidentialité est donc essentielle. Les femmes doivent être convaincues de la discrétion du personnel des FS et surtout, dans la zone B, des agents DBC.

\section{Conclusions et recommandations pour la stratégie DBC.}

La population ne ressent pas la planification familiale comme une priorité. De plus, certains agents DBC se sentent stigmatisés, soupçonnés d'inciter les femmes à la débauche. Pour encourager de façon durable la participation — déjà modeste — de la communauté dans la zone $\mathrm{B}$, il est nécessaire de mettre en œuvre plus d'activités de santé. D'ailleurs, l'activité la plus populaire des agents DBC est la distribution de chloroquine et d'aspirine

Les activités de lutte contre l'excision doivent être menées par d'autres personnes et indépendamment des agents DBC. Ces activités requièrent d'autres approches et d'autres compétences

La participation communautaire dans la zone B n'est pas perceptible. Ni les Comités de Gestion, ni les personnes que nous avons enquêté ne se sentent concernés par les activités DBC. Les Comités de Gestion se concentrent surtout sur la gestion des dépôts pharmaceutiques.

A tous les niveaux la motivation est insuffisante. Les agents DBC ne sont pas régulièrement supervisés. Ils ne maîtrisent pas tous les formulaires de suivi qu'ils doivent remplir. Les ristournes sur les ventes de contraceptifs sont insuffisantes pour être considérées comme source de motivation.

La couverture des agents DBC est faible. Selon l'enquête de 1998, chaque agent DBC a, en moyenne, visité au moins une fois 21 femmes à domicile en 18 mois d'intervention, soit à peine plus d'une nouvelle visite par mois. Seulement $17 \%$ des femmes se souviennent avoir reçu une visite d'un agent DBC. 
La stratégie DBC a modérément réussi à accroître l'accessibilité des contraceptifs. C'est probablement pour cela que la prévalence contraceptive a plus augmenté dans la zone B que dans la zone A, alors que les intentions d'en utiliser à l'avenir y sont restées inchangées.

Les jeunes filles (<25 ans) ont été moins visitées que les femmes plus âgées. Or ce sont les jeunes filles qui sont les plus exposées aux risques liés à l'avortement. Des activités spécifiques à cette tranche d'âge doivent être mises en œuvre.

Ces deux derniers résultats suggèrent que les agents DCB se soient concentrés sur les femmes les plus susceptibles d'acheter des contraceptifs, plutôt que de passer du temps à convaincre celles qui hésitent. La stratégie DBC a donc eu peu d'effet sur les perceptions en matière de PF.

Les agents DBC ont besoin d'être mieux formés. La formation est en soit une source de motivation. Elle est indispensable si l'on souhaite améliorer les perceptions sur la contraception moderne.

Il faut recruter plus d'agents DBC. II y en a actuellement deux par village, quelque soit sa taille et sa population. En conséquent, les agents DBC des grands villages ne peuvent couvrir qu'une petite fraction de leur communauté, et ce de façon superficielle.

Les agents DBC doivent être recrutés pour une durée limitée (18 mois, par exemple). Dans le système actuel, les agents DBC ne sentent pas qu'ils doivent travailler pour garder leur poste (c'est-à-dire pour garder le vélo).

La participation du Ministère de la santé est insuffisante. La stratégie DBC exige beaucoup d'organisation, de formation et de supervision sur le terrain. Cela demande une grande capacité d'écoute et les moyens de réagir rapidement et efficacement. Le Ministère n'a pas su démontrer qu'il avait ces capacités.

La stratégie DBC, telle qu'elle a été testée dans la zone B, n'est ni efficace, ni durable. Elle devra être repensée et sérieusement améliorée avant que l'on puisse envisager sa reproduction ou sa généralisation. 


\section{Avant propos}

Ce rapport d'évaluation de l'intervention de la DSF au Bazega se base sur quatre séries d'enquête : l'enquête quantitative de base (avril - mai 1996), l'enquête qualitative de base (1996) et l'enquête de premier passage (avril - mai 1998) et l'enquête d'évaluation qualitative DBC (novembre 1998). L'intervention elle-même a débuté à partir de la fin de l'année 1996. L'enquête de passage et l'enquête DBC ont donc eu lieu respectivement 18 mois et deux ans après le début des opérations. Il est peut-être trop tôt pour espérer observer des changements significatifs en matière de comportements : ceux-ci devraient pouvoir être mis en évidence par la deuxième enquête de passage (avril - mai 2000). On s'attachera donc à valider les hypothèses du LSC (section 1), à analyser la mise en œuvre de l'intervention (section 2), à tenter de saisir ses effets (section 3) et d'étudier les mérites respectifs des deux stratégies testées dans le Bazèga (section 4), conformément au protocole d'évaluation proposé par l'UERD et validé par les partenaires du LSC (DSF, Population Council, Mwangaza Action et UERD) lors de l'atelier des 17, 18 et 19 février 1998 (cf. protocole d'évaluation). L'UERD a collecté et analysé les données et rédigé ce rapport en toute indépendance. Elle est donc entièrement responsable des données qui y sont présentée et des conclusions qui y sont faites.

Pour répondre aux échéances des bailleurs de fonds, le Population Council a souhaité qu'un rapport soit soumis en décembre 1998 malgré les retards dans la mise à disposition des fonds. C'est pour cela qu'un petit nombre de questions secondaires n'ont pas encore eu de réponses. Ces lacunes toutefois ne changent rien à la validité des conclusions.

L'UERD remercie la Fondation Rockefeller et la Fondation Mellon pour leur soutien financier dans le cadre de cette évaluation. 


\section{Sommaire du rapport d'évaluation}

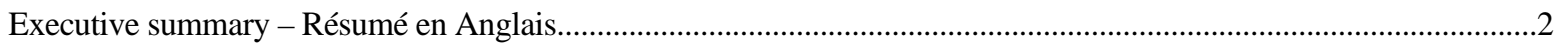

LSC factsheet - Fiches des principaux indicateurs.................................................................................................

Résumé - Principaux résultats et recommandations...............................................................................................11

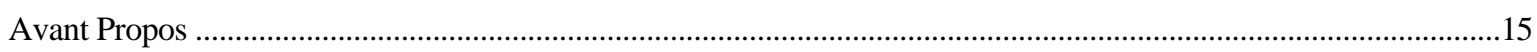

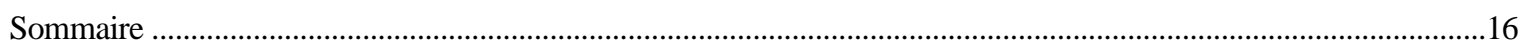

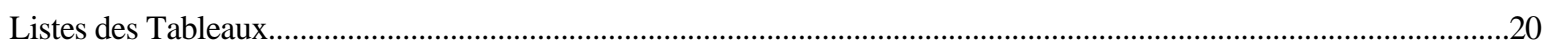

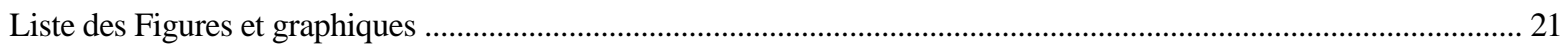

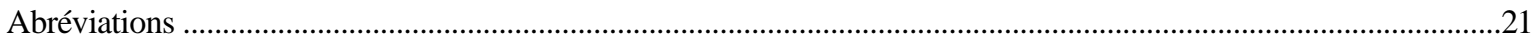

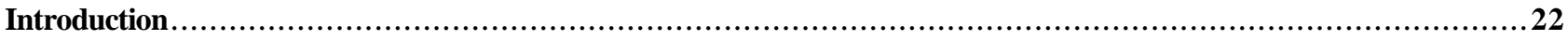

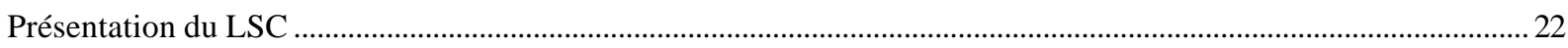

Champ et portée de l'évaluation ............................................................................................................................ 24

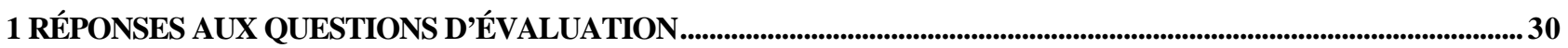

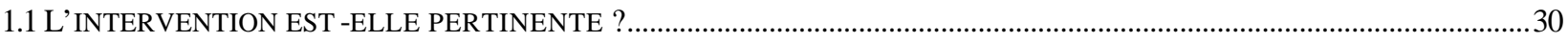

1.1.1 Les hypothèses du programme sont-elles validées ? ......................................................................................... 30

a. Quelles sont les pratiques en matière de fécondité observées dans les quatre zones ?.....................................................31

Q1.1-a1 Les femmes espacent-elles leurs naissances ? . ............................................................................... 31

Q1.1-a2 Les couples (hommes et femmes) expriment-ils le souhait d'espacer les naissances ?.................................34

Q1.1-a3 Les couples (hommes et femmes) expriment-ils le souhait de limiter leur fécondité ? ....................................35

Q1.1-a4 Une femme (un homme) peut-elle (il) convaincre son (sa) conjoint(e) de réguler sa fécondité?......................38

Q1.1-a5 La composition par sexe de la descendance atteinte influence-t-elle le désir d'enfants ?..................................39

Q1.1-a6 Les couples connaissent-ils la manière de maîtriser leur fécondité de façon naturelle ?...................................41

Q1.1-a7 Les couples comprennent-ils comment fonctionnent les méthodes contraceptives modernes ? .....................41

Q1.1-a8 La coutume d'abstinence post-partum est-elle toujours observée ? Quelle est sa durée ? ...............................42

Q1.1-a9 L'intervalle moyen entre deux naissances est-il stable au Bazèga ?.............................................................43

b. Quel est le niveau de la demande de contraception au Bazèga ?..............................................................................44

Q1.1-b1 Que connaissent les couples (hommes et femmes) au sujet de la contraception (méthodes, effets secondaires,

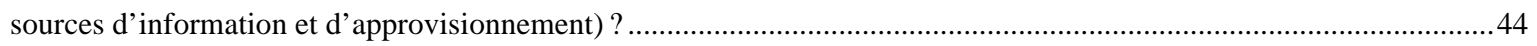

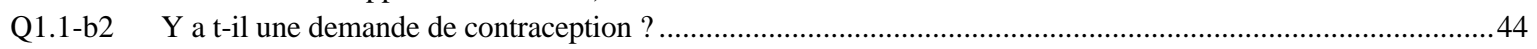

Q1.1-b3 Y a-t-il une demande insatisfaite de méthodes contraceptives modernes ?.....................................................47

Q1.1-b4 Pour quels types de contraceptifs y a-t-il une demande ? ....................................................................47

Q1.1-b5 Y a-t-il une demande pour les méthodes contraceptives traditionnelles ? .................................................47

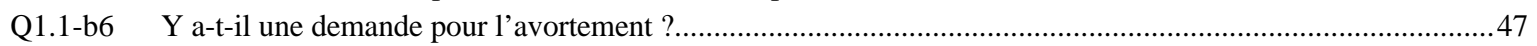

Q1.1-b7 La pilule est-elle une alternative à l'avortement ? .................................................................................47

c. Les CAP en matière de contraception peuvent-elles être modifiées afin d'accroître la demande de contraception ?.............48

Q1.1-c1 Quels sont les obstacles à l'utilisation de la contraception moderne parmi les femmes qui désirent maîtriser

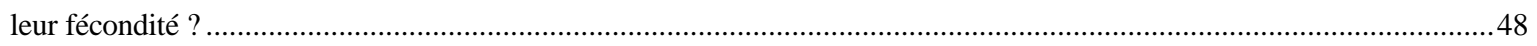

Q1.1-c2 Les façons dont les méthodes de contraception sont perçues limitent-elles leur utilisation ?.........................51

Q1.1-c3 Les méthodes contraceptives sont-elles perçues comme étant efficaces ?....................................................51

Q1.1-c4 Les perceptions sur la contraception varient-elles avec l'âge, le sexe, la religion ?......................................51

Q1.1-c5 Les méthodes contraceptives modernes sont-elles culturellement acceptables ?.........................................53

Q1.1-c6 L'intervention peut-elle modifier ces perceptions en trois ans ? ............................................................55

Q1.1-c7 La modification des perceptions entraîne-t-elle une augmentation de l'utilisation des contraceptifs ?............55

Q1.1-c8 Les migrations et la proximité de Ouagadougou influencent-elles l'utilisation de la contraception ?..............55 
d. La prévalence de l'utilisation de la contraception est-elle faible parce que l'offre est inadéquate ?...................................57

Q1.1-d1 Quelle était la prévalence contraceptive avant l'intervention ?.................................................................57

Q1.1-d2 Le coût des contraceptifs a-t-il un effet sur leur niveau d'utilisation ?.........................................................59

Q1.1-d3 L'utilisation des contraceptifs est-elle associée à la distance de la formation sanitaire ?.................................60

Q1.1-d4 Y a-t-il une offre pour d'autres méthodes de maîtrise de la fécondité ?........................................................60

1.1.2 La situation au Bazèga justifie-t-elle une intervention en planification familiale? 60

a. La fécondité est-elle plus élevée au Bazèga que dans l'ensemble du Burkina Faso rural ?

Q1.2-a1 Le taux de fécondité global dans le Bazèga est-il significativement plus élevé que dans l'ensemble du Burkina Faso rural ?61

Q1.2-a2 Les connaissances, aptitudes et pratiques en matière de PF sont-elles moins favorables à une baisse de la fécondité dans les zones LSC que dans l'ensemble du Burkina Faso rural ?.....

Q1.2-a3 La demande non satisfaite de PF est-elle plus importante dans les zones LSC que dans l'ensemble du Burkina Faso rural ?63

Q1.2-a4 La prévalence de l'utilisation de la contraception est-elle plus faible dans les zones LSC que dans l'ensemble du Burkina Faso rural ?

b. La situation démographique et sanitaire est-elle plus difficile au Bazèga que dans l'ensemble du Burkina Faso rural ? ......63 Q1.2-b1 La mortalité infantile est-elle plus élevée dans les zones LSC que dans l'ensemble du Burkina Faso rural ?...65 Q1.2-b2 La mortalité infanto-juvénile est-elle plus élevée dans les zones LSC que dans l'ensemble du Burkina Faso rural ?

\subsubsection{Couverture de l'intervention.}

Q2.1-a1 Quelle est la proportion de ménages de la zone B qui a été visitée par les agents DBC ?.....

Q2.1-a2 Quelle est la proportion d'individus qui ont visité leur FS au cours des douze derniers mois ? Sont-ils allés à une autre formation sanitaire?...

Q2.1-a3 Pour quels motifs ces femmes ont-elles visité leur FS ?.....

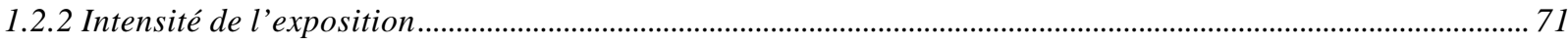

Q2.2-a1 Est-ce que l'enquêtée connaît son agent DBC (Zone B)? .............................................................................71

Q2.2-a2 Combien de fois l'agent DBC a-t-il visité l'enquêtée ces douze derniers mois ?..........................................72

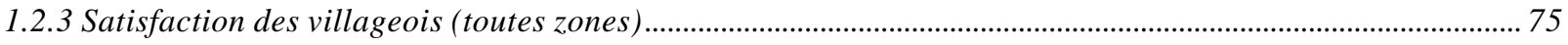

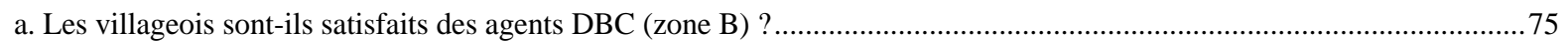

Q2.3-a1 Les enquêtés sont-ils satisfaits du travail des agents DBC dans leur village (zone B) ?...............................75

Q2.3-a2 Tous les enquêtés souhaiteraient-ils devenir un agent DBC dans leur village (zone B) ?.............................77

b. Les villageois sont satisfaits de l'amélioration de leurs formations sanitaires ? (toutes zones) .........................................78

1.3 L'INTERVENTION A-T-ELLE EU UN EFFET? .79

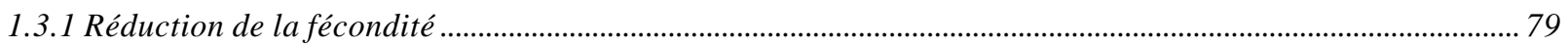

a. Le niveau de fécondité a-t-il significativement baissé entre l'enquête de base et les autres passages ?...............................79

Q3.1-a1 La réduction de la fécondité est-elle plus forte dans les zones d'intervention ?...........................................79

b. Par quels mécanismes cette réduction s'est-elle opérée (ou pourrait-elle s'opérer à l'avenir) ? ........................................79

Q3.1-b1 La prévalence contraceptive a-t-elle plus augmenté dans les zones B et/ou A que dans la zone C ? ..............80

Q3.1-b2 La demande contraceptive a-t-elle plus augmenté dans les zones B et/ou A que dans la zone C ? ................81

Q3.1-b3 La réduction de la fécondité est-elle attribuable à l'intervention ? ................................................................83

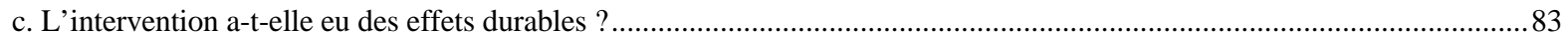

Q3.1-c1 Y a t-il une augmentation de l'utilisation de la contraception chez les jeunes gens ?....................................83

Q3.1-c2 Y a t-il eu des changements favorables dans les perceptions de la PF ? ...................................................84

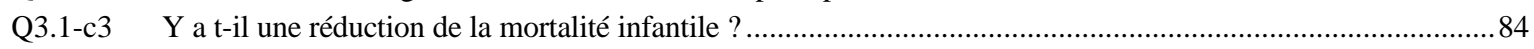


Q4.1-a1 L'augmentation de la demande de contraception dans la zone B est-elle supérieure à celle de la zone A, ellemême supérieure à celle de la zone $\mathrm{C}$ ?.

Q4.1-a2 Les perceptions sur la PF ont-elles changé plus favorablement dans la zone B que dans la zone A et en zone

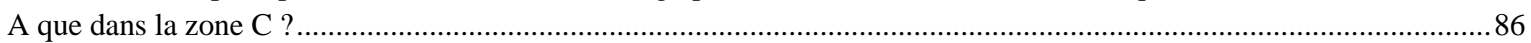

Q4.1-a3 La variation de la demande insatisfaite pour la contraception sont-elles différentes entre les zones A, B et C ?

1.4.2 L'approche communautaire est-elle plus efficace qu'une distribution basée dans les formations sanitaires ?

Q4.2-a1 La présence des agents DBC est-elle associée à une augmentation de la connaissance des méthodes contraceptives?.

Q4.2-a2 La présence des agents DBC est-elle associée à une augmentation de la prévalence de l'utilisation des méthodes contraceptives?

1.4.3 L'approche communautaire est-elle meilleure en termes de coût-efficacité ?..... 89

a. Est-ce que la formule payante de recouvrement des coûts constitue une entrave à l'utilisation de la contraception ?..........89

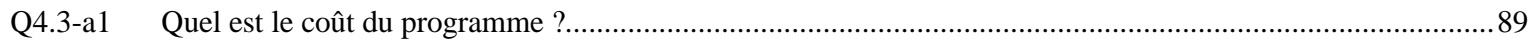

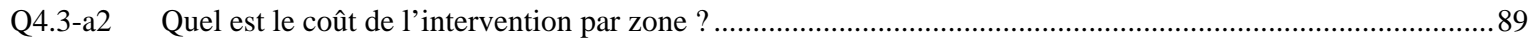

Q4.3-a3 Combien les enquêtées déclarent-elles être prêtes à payer pour des contraceptifs ? (voir aussi Q1.1-d2) ......89

Q4.3-a4 Est-ce que le prix des contraceptifs est un facteur de poids dans la décision de recourir à la contraception ?.90

Q4.3-a5 Les méthodes contraceptives moins chères sont-elles préférées aux méthodes plus onéreuses ? ...................90

Q4.3-a6 Les enquêtés font-ils confiance aux agents DBC et aux comités de gestion ?................................................90

Q4.3-a7 La formule de recouvrement des coûts par les agents DBC est-elle plus efficace ?......................................90

b. Les enquêtés (es) préfèrent-ils (elles) s'approvisionner en contraceptifs auprès des agents DBC de leur village plutôt qu'à

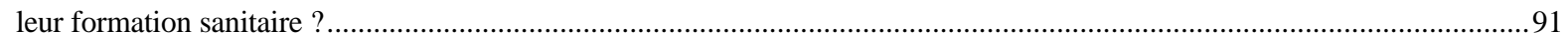

Q4.3-b1 Y a t-il des individus qui connaissent l'agent DBC de leur village et qui continuent à s'approvisionner à la FS ?

Q4.3-b2 Y a t-il des individus qui connaissent l'agent DBC de leur village et qui continuent à s'approvisionner ailleurs ?

Q4.3-b3 Y a t-il des enquêtés qui affirment vouloir utiliser des méthodes contraceptives modernes mais qui ne savent pas où s'approvisionner?

1.4.4 L'approche communautaire est-elle plus durable?

a. Les villageois perçoivent-ils la maîtrise de leur fécondité comme un besoin urgent et prioritaire qui doit être résolu par la collectivité ?.

Q4.4-a1 Quels problèmes sont-ils perçus comme les plus urgents à résoudre ? .....................................................95

Q4.4-a2 Quels problèmes de santé sont-ils perçus comme les plus urgents à résoudre ? ..........................................96

Q4.4-a3 La fécondité non maîtrisée constitue-t-elle un problème qui peut être résolu par la communauté ? ...............96

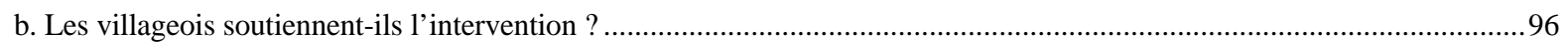

Q4.4-b1 Que connaissent les enquêtés des activités de l'agent DBC ? ..............................................................96

Q4.4-b2 Quels sont les avantages matériels et sociaux que les villageois retirent de l'intervention ?...........................97

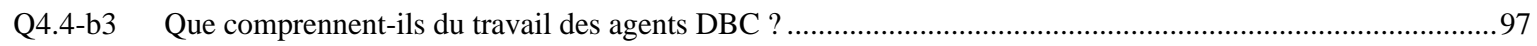

Q4.4-b4 Que comprennent-ils du rôle du comité de gestion dans les activités de DBC ? ..........................................97

Q4.4-b5 Les enquêtés font-ils confiance aux agents DBC ?.........................................................................97

Q4.4-b6 Les enquêtés font-ils confiance au comité de gestion ? .........................................................................98

Q4.4-b7 Les villageois sont-ils motivés (soutien et engagement) ? .....................................................................98

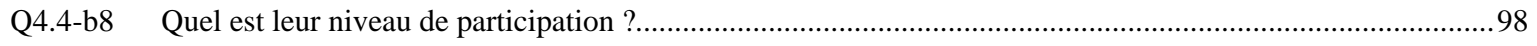

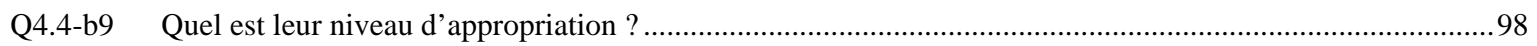

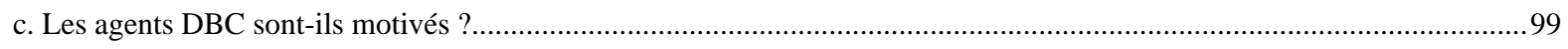

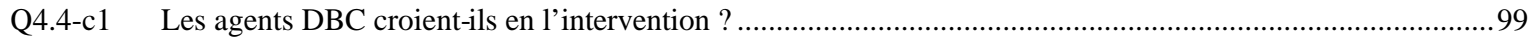

Q4.4-c2 Quels sont les problèmes rencontrés par les agents DBC dans le cadre de leurs activités ?...........................99

Q4.4-c3 Quelles sont les autres activités et responsabilités des agents DBC ? disponibilité pour les activités DBC.100

Q4.4-c4 Combien de temps les agents DBC consacrent-ils à l'intervention ?.......................................................100

Q4.4-c5 Que comprennent les agents DBC de leurs tâches et de leur rôle ? .............................................................101

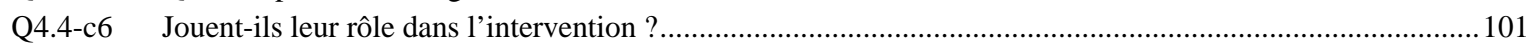

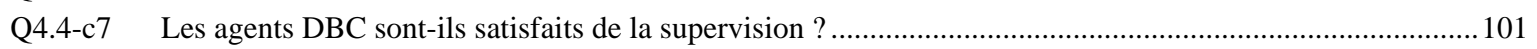

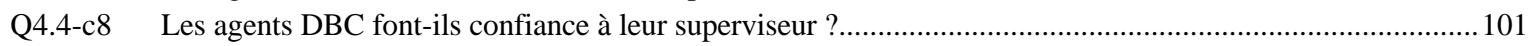


Q4.4-c9 Quelle est la fréquence des rapports et des réunions avec laquelle les agents DBC rencontrent leurs superviseurs pour leur faire le point ? (ordre du jour et contenu des réunions)..........................................................101 Q4.4-c10 Quelle est la fréquence avec laquelle les agents DBC parlent à leur comité de gestion de leurs activités ?......102

Q4.4-c11 Les agents DBC savent-ils à qui ils doivent rendre des comptes ?..............................................................102

Q4.4-c12 A qui les agents DBC rendent-ils effectivement compte ? ..........................................................................102

Q4.4-c13 La ristourne sur les produits vendus constitue-t'elle une motivation ?.........................................................102

Q4.4-c14 Quel est le degré de remplissage des instruments de gestion ? (qualité) .....................................................102

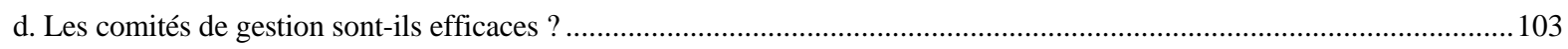

Q4.4-d1 Les membres du comité de gestion croient-ils en l'intervention ?.................................................................103

Q4.4-d2 Quels sont les problèmes rencontrés par les membres du comité de gestion ?.............................................103

Q4.4-d3 Quelles sont les autres activités et responsabilités des membres de comités de gestion ?.............................103

Q4.4-d4 Combien de temps les membres du comité de gestion consacrent-ils à l'intervention DBC ?........................104

Q4.4-d5 Quelle est la fréquence de réunion du comité autour des activités DBC ?.................................................105

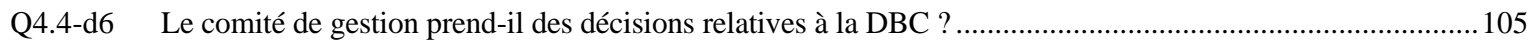

Q4.4-d7 Le comité de gestion rend-il des comptes aux villageois ?..........................................................................105

Q4.4-d8 Quelles sont les dernières décisions prises et mises en œuvre relatives aux activités DBC ?........................106

Q4.4-d9 L'activité est-elle intégrée au plan d'action du comité de gestion ? .........................................................106

e. Le personnel des formations sanitaires est-il motivé par la participation des agents DBC et des comités de gestion ?.....106

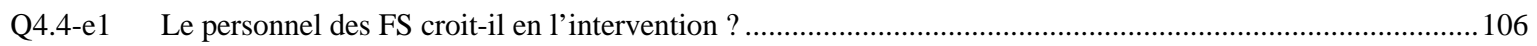

Q4.4-e2 Le personnel des FS croit-il que l'on peut augmenter l'utilisation de la contraception ? ..............................107

Q4.4-e3 Le personnel des FS croit-il que la participation des agents DBC peut augmenter l'utilisation de la

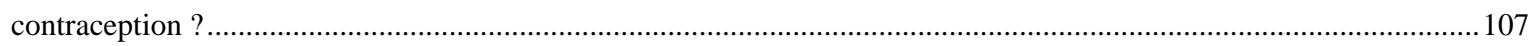

Q4.4-e4 Le responsable de la PF de la FS croit-il que la formule DBC devrait être étendue à d'autres zones ?..........107

Q4.4-e5 Le personnel de santé a-t-il déjà entendu parler de problèmes dans son aire de travail concernant les agents

DBC ou le comité de gestion ? .......................................................................................................... 107

Q4.4-e6 Le personnel de santé a-t-il déjà entendu parler de problèmes dans son aire de travail concernant les comités

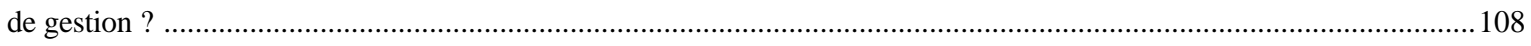

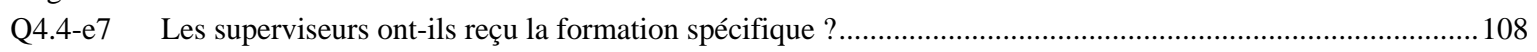

Q4.4-e8 Les superviseurs ont-ils été mutés depuis le début de l'intervention ? .....................................................108

Q4.4-e9 Quel est le niveau de soutien, d'engagement du personnel auprès des agents DBC ? ..................................108

f. Les responsables de district sont-ils motivés par l'approche communautaire ?........................................................... 109

Q4.4-f1 Les responsables des districts croient-ils que l'on peut augmenter l'utilisation de la contraception ?...........109

Q4.4-f2 Les responsables des districts croient-ils que la participation des agents DBC peut augmenter l'utilisation de

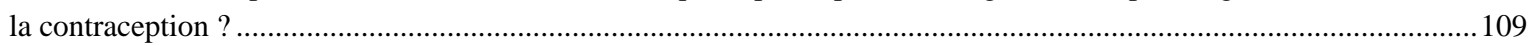

Q4.4-f3 Les responsables des districts croient-ils que la formule DBC devrait être étendue à d'autres districts ?.....109

Q4.4-f4 Les responsables des districts ont-ils déjà entendu parler de problèmes concernant les agents DBC ?.........109

Q4.4-f5 Les responsables des districts ont-ils déjà entendu parler de problèmes concernant les comités de gestion ?109

Q4.4-f6 Les responsables de districts ont-ils reçu la formation spécifique ?...........................................................109

Q4.4-f7 Les responsables de districts ont-ils été mutés depuis le début de l'intervention ? .......................................109

Q4.4-f8 Quel est le niveau de soutien, d'engagement des responsables de district auprès des agents DBC ?............109

Q4.4-f9 L'activité DBC est-elle prévue dans le plan d'action du district ? ...........................................................109

2.1.1 Principe $D B C$ :

2.1.2 Logistique, encadrement

2.1.3 Au niveau communautaire 


\section{Tableaux}

Tableau 1 : Intervalle intergénésique. 33

Tableau 2 : Nombre idéal de garçons et de filles. 36

Tableau 3 : Descendance finale et nombre d'enfants survivants, selon le sexe. 37

Tableau 4 : Connaissances et pratiques de la contraception moderne. 42

Tableau 5 : Evolution de l'intervalle intergénésique. 44

Tableau 6 : Besoins en planification familiale en 1996 (en \%). 45

Tableau 7 : Connaissance et utilisation de la contraception des femmes mariées selon leur religion. 52

Tableau 8: proportion des femmes de 15 à 49 ans qui ont séjourné à Ouaga lors des 12 derniers mois, selon la zone, l'âge et l'utilisation d'une méthode moderne de contraception. 56

Tableau 9: probabilité pour une femme de 15-49 ans d'avoir utilisé une méthode moderne de contraception selon les séjours à Ouaga. $\quad 56$

Tableau 10 : Prévalence contraceptive par Zone, 1996.

Tableau 11 : Indicateurs de fécondité par zones. $\quad 62$

Tableau 12 : Principaux indicateurs de santé, zones LSC. 64

Tableau 13 : Statistiques de couvertures et de rendement DBC par FS . 67

Tableau 14 : Proportion des femmes ayant reçu au moins une visite selon le groupe d'âge. 68

Tableau 15: Fréquentation des FS dans les quatre zones. 69

Tableau 16 : Motifs de fréquentation des FS de reference au cours des 12 derniers mois, par zone. 71

Tableau 17 : Frequences et distribution des visites ADBC par formation sanitaire. 72

Tableau 18 : Couverture des différentes activités des ADBC. 77

Tableau 19 : Proportion de femmes mariées utilisant une méthode de PF moderne. 80

Tableau 20 : Intention d'utiliser une méthode contraceptive moderne par zone en 1996 et 1998.

Tableau 21 : Prévalence contraceptive chez les jeunes femmes (15-24 ans) à ceux de l'ensemble des femmes en union, par zone en 1996 et 1998.

Tableau 22 : taux de mortalité par zone et par année. 84

Tableau 23 : Proportion de femmes mariées ayant entendu parler de contraception, 1996 et 1998.

Tableau 24 : conditions de durabilité de la stratégie DBC. 95

Tableau 25 : Stratégie DBC - points forts et points faibles 110

Tableau 26 : Stratégie DBC - problèmes rencontrés. 110 


\section{Figures et graphiques}

Figure 1 : Les hypothèses de l'intervention DBC-PF au Bazèga 30

Figure 2 : Concept et durée d'espacement des naissances, selon le sexe. 34

Figure 3 : Désir d'enfant supplémentaire selon l'âge et le nombre d'enfants survivants. 35

Figure 4 : Probabilité pour une femme de vouloir un autre enfant, selon le nombre de garçons et de filles survivants.

Figure 5 : Besoins des femmes en matière de planification familiale selon leur âge. 46

Figure 6 : Raisons invoquées par les femmes qui ne pratiquent pas la contraception. 49

Figure 7 : Obstacles à l'utilisation de la contraception. $\quad 50$

Figure 8: Opinions sur la contraception selon l'âge et la religion. 53

Figure 9 : Méthodes contraceptives utilisées par les femmes mariées pratiquant la PF. 59

Figure 10 : Taux de fécondité par âge dans le Bazèga, à Ouaga et en zone rurale. 61

\section{Abréviations}

CG Comité de gestion

DBC Distribution à base communautaire

DSF Direction de la santé de la famille

EDS Enquête démographique et de santé (DHS, Demographic and Health Survey)

FS Formation sanitaire

LSC Laboratoire de santé communautaire

PF Planification familiale

UERD Unité d'enseignement et de recherche en démographie 


\section{Introduction}

\section{Présentation du LSC}

Au cours des trente dernières années, le Burkina Faso a fait d'énormes progrès dans l'amélioration des conditions de santé des populations. En effet les indicateurs de base de l'état de santé (mortalité infantile par exemple) ont baissé. En outre les infrastructures sanitaires du pays ont été améliorées. Cependant, malgré ces réalisations, la mortalité infantile reste élevée. Elle est de 113 pour 1000 naissances vivantes dans les zones rurales. La mortalité maternelle est aussi très élevée : 566 pour 100000 naissances vivantes. Il en est même de la fécondité. Le nombre moyen d'enfants par femme est de 6,9 mais varie entre 4,7 enfants à Ouagadougou et 7,3 en milieu rural.

Le programme de planification familiale a démarré au Burkina Faso seulement en 1986. Comme l'ensemble des services de santé, il est concentré dans les zones urbaines où il a connu beaucoup plus de succès. L'Enquête Démographique et de Santé (EDS/BF - 1993) révèle par exemple un taux de prévalence de la contraception moderne de $17 \%$ en milieu urbain contre moins de $2 \%$ en milieu rural. Ceci traduit une concentration des services de santé et de planification familiale dans les villes. La prestation de services de qualité aux populations rurales constitue sans aucun doute, un grand défi pour les autorités burkinabé.

C'est dans ce contexte que le Gouvernement burkinabé a entrepris une restructuration totale de son système sanitaire, caractérisée par une décentralisation poussée des services. Pour ce faire, il a reçu de la Banque Mondiale, une subvention de 35 millions de dollars destinée à l'aider à mener la réforme nécessaire à une amélioration significative de la qualité des services fournis aux populations. Cette réforme basée sur l'approche «district», est largement inspirée des recommandations d'une série d'études conduites pour évaluer le programme national de santé (1980-1990) et celui de la santé familiale en particulier (1988-92). Les résultats de ces études révèlent principalement des problèmes liés à la disponibilité, à l'accessibilité et à la qualité des services de santé.

Toutefois en ce qui concerne la planification familiale, il n'est pas évident que disponibilité et accessibilité signifient nécessairement utilisation, particulièrement en zone rurale. En d'autres termes, l'on ne sait pas s'il y a effectivement une demande pour les services de planification familiale. 
Ainsi, le Gouvernement du Burkina Faso a entrepris, à partir de 1995 de tester une nouvelle approche visant à amoindrir les problèmes d'accessibilité et de qualité des services dans une province rurale du Burkina Faso, le Bazèga. Dans la zone du Bazèga, de Ministère de la Santé (MS) se propose de conduire un programme d'expérimentation et d'essai de moyens réalisables pour rendre accessible aux populations rurales des services de soins de santé complets et de haute qualité.

Un système sanitaire efficace et à large couverture est celui qui facilite l'accès de tous les couples aux services offerts. Cependant un tel système ne sera vraiment efficace que s'il existe une demande appréciable de contraceptifs ainsi qu'un système de référence des client(es) à des centres de santé. Il n'existe pas pour le moment au Burkina Faso de moyen pour identifier les clientes potentielles dans les zones rurales et de les référer aux services existants. Compte tenu de cette situation, le programme national de Planification familiale voudrait tester comme moyens d'atteindre un nombre assez important de clientes potentielles dans les zones rurales, des stratégies de distribution à base communautaire leur fournissant les services à domicile ou leur recommandant des structures sanitaires auxquelles elles peuvent s'adresser.

L'étude de recherches opérationnelles proposée a ainsi pour objectif d'aider le Ministère de la santé à trouver une stratégie appropriée et à moindre coût de prestation de services de planification familiale qui cadre avec le nouveau programme de reforme de la santé au niveau national. Cette étude de recherches opérationnelles se propose donc d'exécuter et de tester un programme de prestation de services de santé et de planification familiale à base communautaire qui soit unique et innovateur. Si le test s'avère couronné de succès, le programme sera étendu à d'autres parties du pays.

L'étude cherche à tester deux formules. Dans la première formule, les services sont offerts uniquement dans les centres de santé qui ont été rénovés et le personnel recyclé. Une gamme plus variée de méthodes contraceptives y a été rendue disponible pour les clientes. La deuxième formule mise en œuvre dans une deuxième zone géographique comporte également le recyclage du personnel et l'amélioration des structures existantes mais comporte en outre, l'intervention des distributeurs à base communautaire (DBC) au niveau des villages. Ces agents DBC sont des volontaires formés pour faire des visites à domicile, vendre des contraceptifs (condoms, mousses et réapprovisionnement en pilules), recommander aux clientes des centres de santé auxquels elles peuvent s'adresser pour d'autres services et également offrir quelques services de santé tels les sels de réhydratation par voie orale (SRO) en cas de diarrhée. Chaque agent DBC a reçu une bicyclette pour ses déplacements et un sac pour le transport des provisions. 
Tester l'efficacité de ces deux formules et les comparer à une zone témoin dans laquelle le programme gouvernemental habituel suit son cours, devrait fournir des données pour une évaluation raisonnable de l'utilité de la rénovation des centres de santé et/ou du rajout de DBC. En développant ces formules, l'hypothèse sous-jacente est que l'intensité des rencontres entre les agents mobiles et les villageois aura un impact sur la demande et l'utilisation des services.

Le Gouvernement burkinabé voit à travers cette étude, la première étape du développement d'un «Laboratoire de Santé Communautaire». Il s'agit d'une station de recherche capable de jouer un rôle similaire à celui du Centre de recherche en santé de Navrongo au Ghana ; c'est-à-dire créer un environnement où les activités systématiques et détaillées peuvent être utilisées pour évaluer une gamme de nouvelles approches innovatrices dans la prestation des services de santé et de population. Les résultats de telles études expérimentales peuvent être utilisées par les décideurs politiques et les concepteurs de programmes pour identifier les approches susceptibles d'être couronnées de succès avant leur utilisation à grande échelle et leur exécution au niveau national.

L’objectif général du projet de Laboratoire de Santé Communautaire (LSC) est de contribuer au développement d'une stratégie efficace et durable de prestation de services de santé et de planification familiale au niveau rural au Burkina Faso. Une telle stratégie permettra au Ministère de la Santé d'étendre à l'ensemble du pays les services de santé et de planification familiale à partir des communautés rurales.

L’objectif immédiat est de déterminer si :

1. la rénovation des centres de santé et l'élargissement des possibilités de choix de méthodes (Formule A) ou

2. la rénovation des centres de santé, l'élargissement des possibilités de choix de méthodes et le rajout d'une composante DBC (Formule B)

aboutissent à des résultats différents et significatifs en termes d'aide aux couples à satisfaire leurs besoins en matière de procréation.

\section{Champ et portée de l'évaluation}


En accord avec le protocole d'évaluation proposé par l'UERD et discuté, amendé et validé par les partenaires institutionnels du LSC lors de l'atelier des 17, 18 et 19 février 1998, le présent rapport provisoire apporte les premiers éléments de réponses à quatre types de question.

1) L'intervention est-elle pertinente ? En d'autres termes, (1) les hypothèses sont-elles valides, (2) la situation démographique et sanitaire au Bazèga justifie-t-elle la mise en œuvre de ce programme de planification familiale ?

2) La mise en cuvre de l'intervention est-elle satisfaisante ? Quelle est la proportion de la population effectivement couverte ? Quelle est l'intensité de l'exposition des individus aux différentes composantes du programme de PF ? Comment le programme est-il perçu par la communauté en général (les villageois), les participants (comités de gestion, agents DBC, personnel des formations sanitaires), la population cible (hommes et femmes en âge de procréer) et les bénéficiaires (utilisateurs de contraceptifs modernes) ?

3) L'intervention a-t-elle eu un effet sur les perceptions, attitudes et comportements en matière de fécondité ? Si des changements statistiquement significatifs sont observés, par quels mécanismes les explique-t-on?

4) Quelle est la stratégie la plus efficace, la plus durable, la moins coûteuse parmi les deux formules expérimentées?

\section{Les évaluateurs}

Toutes les institutions partenaires du LSC (DSF, Population Council, Mwangaza et UERD) ont participé à l'élaboration du cadre de l'évaluation. Ils ont validé le protocole d'évaluation préparé par l'UERD après s'être assuré que leurs questions y figuraient bien que la méthodologie proposée soit conforme à leurs préoccupations. Nous avons privilégié les méthodes d'évaluation simples afin de faciliter la participation de non-statisticiens au processus d'évaluation.

Afin d'éviter tout reproche de partialité, les chercheurs de l'UERD ont conduit les travaux de terrain et ont coordonné l'analyse des données en toute indépendance, grâce notamment au soutiens des fondations Rockefeller et Mellon. Conformément au protocole, les évaluateurs n’ont aucune responsabilité opérationnelle dans l'intervention et n'ont aucun lien, financier ou hiérarchique, avec une des institutions qui participent à l'intervention. 


\section{Données et méthodes}

L'évaluation se base sur une série complete et complementaires de données collectées par l'UERD. Ces opérations de collecte sont les suivantes :

1. cartographie ;

2. énumération de toutes les concessions des quatre zones (base de sondage du panel) ;

3. enquête de base (avril-juin 1996) ;

4. enquête qualitative de base (1997) ;

5. enquête de passage (avril-juin 1998);

6. enquête qualitative d'évaluation de la stratégie DBC (novembre 1998).

\section{Méthodes quantitatives (enquête de bas et enquête de passage).}

L'échantillon est constitué de 1600 concessions, à raison de 400 concessions par zones, tirées au hasard sur la base des liste d'énumération. Dans chaque concession tirée, un enquêteur administre à tous les hommes mariés et toutes les femmes âgées de 15 à 49 ans un questionnaire. De plus, un questionnaire propre à la concession (1996) et à chaque ménage (1996 et 1998) est rempli.

Les enquêtes quantitatives ont pour objectifs de suivre dans le temps a la fois (1) l'évolution d'indicateurs de façon transversale (par ex ; augmentation de la prévalence contraceptive dans les différentes zones) et (2) mesurer l'effet de l'intervention au niveau des individus (suivi longitudinal, ou panel)

L'enquête par «panel» consiste en la répétition de l'enquête sur un même échantillon à certains intervalles de temps ${ }^{5}$. Elle est généralement recommandée «dans les opérations locales, sans souci de représentation régionale ou nationale, pour l'étude d'un problème spécifique à caractère expérimental, où l'on suit une cohorte d'individus». Cela permet de suivre les modifications que l'échantillon subit au cours du temps. L'option prise pour le suivi du panel dans le LSC est la dépendance partielle des passages : les enquêteurs retournent sur le terrain avec la liste nominative des individus du passage précédent.

La comparaison transversale nécessite des échantillons comparables d'un passage à l'autre (notamment au niveau des structures par âge). Pour éviter que le panel vieillisse avec le temps, toutes les femmes de 15 à 49 ans sont enquêtées à chaque passage. Ainsi, les sortie des femmes de l'échantillon (décès et viellissement et départ des filles suite a leur mariage) sont «compensées » par les filles de la concession qui atteignent 15 ans, ou celles qui y entrent par mariage («femmes nouvelles »).

\footnotetext{
${ }^{5}$ Cette introduction sur les problèmes liés « aux enquêtes à panel » s'inspire de Tabutin D., 1984, La collecte des données en démographie: méthodes, organisation et exploitation, Ordina Editions, Liège, 258 p.
} 
La définition du ménage retenue lors de l'enquête de base a été strictement respectée: " un ménage correspond à une personne ou un ensemble de personnes apparentées ou non, qui vivent ensemble dans la même maison, mettent en commun leurs ressources et satisfont en commun l'essentiel de leurs besoins alimentaires ou vitaux (Manuel de l'enquêteur, Enquête de base du LSC) ». En règle générale, un ménage est constitué d'un homme (chef de ménage), de ses épouses, de ses enfants et dépendants. Ainsi, dans une même concession on peut avoir plusieurs ménages. Exemple: si dans une concession on dénombre trois hommes mariés, on dira qu'on a trois ménages distincts avec comme membres, ceux que le chef de ménage déclarera être « sous sa coupe».

Tableau 1 : Echantillon des enquêtes et de Panel, 1996-1998.

\begin{tabular}{|c|c|c|c|}
\hline & \multicolumn{2}{|c|}{ Echantillons pour analyses transversales } & \multirow{2}{*}{$\begin{array}{l}\text { Echantillon longitudinal } \\
\text { Panel }{ }^{1} \\
\end{array}$} \\
\hline & 1996 & 1998 & \\
\hline Femmes (15-49 ans) & 2804 & 2532 & 1938 \\
\hline Hommes (chef de ménage) & 1872 & 2084 & 1766 \\
\hline
\end{tabular}

1. individus présents en 1996 et en 1998

Méthodes qualitatives (enquête qualitative de base et enquête qualitative d'évaluation de la stratégie $D B C$.

Le Tableau 2 et le Tableau 3 présentent les entretiens que l'UERD a mené en matière de santé de la reproduction dans le cadre du LSC. 
Tableau 2 : Catégories de groupes pour les entretiens dirigés (1997)

\begin{tabular}{|c|c|c|c|c|c|c|c|c|}
\hline & \multicolumn{7}{|c|}{ Nombres d'entretiens (nombre de participant à l'entretien) } & \multirow[b]{3}{*}{ total } \\
\hline & \multicolumn{3}{|c|}{ Femmes } & \multicolumn{3}{|c|}{ Hommes } & \multirow{2}{*}{$\begin{array}{l}\text { groupes } \\
\text { spécif. }^{6}\end{array}$} & \\
\hline & $\begin{array}{l}\text { jeunes } \\
\text { célibat. }\end{array}$ & mariées & âgées & $\begin{array}{l}\text { jeunes } \\
\text { célibat. }\end{array}$ & mariés & âgés & & \\
\hline Village (Zone) & un (8) & un (10) & un (9) & un (7) & un (7) & un (10) & quatre & $\operatorname{dix}$ \\
\hline Bonsrima (A) & un (12) & un (11) & un (9) & un (9) & un (6) & un (8) & quatre & dix \\
\hline Kienfangue (B) & un (12) & un (13) & un (7) & un $(8)$ & un $(8)$ & un (7) & un & sept \\
\hline Kalzi (B) & un (11) & un (7) & un (12) & un (8) & un (7) & un (11) & & $\operatorname{six}$ \\
\hline Bere $(C)$ & un (9) & un $(8)$ & un (13) & un (8) & un (8) & un (9) & & $\operatorname{six}$ \\
\hline \multicolumn{9}{|l|}{ Kuizili (D) } \\
\hline Total & $\operatorname{cinq}(52)$ & cinq (49) & $\operatorname{cinq}(50)$ & $\operatorname{cinq}(40)$ & $\operatorname{cinq}(36)$ & $\operatorname{cinq}(35)$ & neuf & trente-neut \\
\hline
\end{tabular}

${ }^{6}$ Les groupes spécifiques sont composés de participants sélectionnés sur la base de la religion (catholiques, protestants, musulmans, sunnites) ainsi que deux groupes d'élèves de l'enseignement secondaire 
Tableau 3 : Entretiens d'évaluation de la stratégie DBC (1998).

\begin{tabular}{|c|c|c|c|c|c|c|}
\hline & $\begin{array}{l}\text { personnel } \\
\text { entretiens } \\
\text { individuels }\end{array}$ & $\begin{array}{l}\text { comité gestion } \\
\text { entretiens } \\
\text { groupe }\end{array}$ & $\begin{array}{l}\text { DBC } \\
\text { entretiens } \\
\text { individuels }\end{array}$ & village & $\begin{array}{l}\text { communautés } \\
\text { entretiens } \\
\text { groupe }\end{array}$ & $\begin{array}{l}\text { autres } \\
\text { entretiens } \\
\text { individuels }\end{array}$ \\
\hline \multicolumn{7}{|l|}{$\begin{array}{l}\text { Formation } \\
\text { sanitaire }\end{array}$} \\
\hline \multirow[t]{7}{*}{ Vipalogo } & major & membres $(18 / 22)$ & $\begin{array}{l}\text { Ilboudo Kuilega } \\
\text { (AV) }\end{array}$ & Goumoogo & & président $\mathrm{CG}$ \\
\hline & accouch. aux. & & Ilboudo Roger & Vipalogo & & \\
\hline & & & Ilboudo Aïssa (AV) & Nabelin & Chef +3 Vieux & \\
\hline & & & Barry Yero (ASC) & Tiyardo & & \\
\hline & & & Nikiema Paratsida & Vipalogo & & \\
\hline & & & Ilboudo Deborah & Yelou & & accoucheuse vil. \\
\hline & & & Koanda Seydou & Absendo & & \\
\hline \multirow[t]{4}{*}{ Komki } & Major & membres $(8 / 13)$ & $\begin{array}{l}\text { Rouamba Jean Paul } \\
\text { (ASC) }\end{array}$ & Bargo & & \\
\hline & accouch. aux. & & Ilboudo Justine & Viou & & \\
\hline & & & $\begin{array}{l}\text { Bonkoungou Poko } \\
\text { (AV) }\end{array}$ & Yaoghin & & \\
\hline & & & Kola Marcel (ASC) & Yaoghin & & \\
\hline Tintilou & & & $\begin{array}{l}\text { Ouedraogo } \\
\text { Djamilatou }\end{array}$ & Itaoua & & \\
\hline \multirow[t]{8}{*}{ Komsilga } & accouch. aux. & membres $(12 / 30)$ & Nonguierma Jeanne & Bangma & Chef +7 pers. & accoucheuse vil \\
\hline & & & $\begin{array}{l}\text { Nikiema Madeleine } \\
\text { (AV) }\end{array}$ & Segdin & & \\
\hline & & & Kabore Marcelline & Tansega & & \\
\hline & & & $\begin{array}{l}\text { Zoungrana Michel } \\
\text { (ASC) }\end{array}$ & Tansega & & \\
\hline & & & $\begin{array}{l}\text { Tiemtore Pascal } \\
\text { (ASC) }\end{array}$ & Segdin & & \\
\hline & & & Yanogo Rose (AV) & Goumsi & & \\
\hline & & & $\begin{array}{l}\text { Kabore François Y. } \\
\text { (PSP) }\end{array}$ & Goumsi & & \\
\hline & & & $\begin{array}{l}\text { Ouedraogo } \\
\text { Ambroise }\end{array}$ & Kalzi & & \\
\hline Total entretiens & & 6 & 20 & & & 3 \\
\hline
\end{tabular}




\section{REPONSES AUX QUESTIONS D'EVALUATION}

\subsection{L'intervention est-elle pertinente?}

La pertinence de l'intervention est évaluée sous deux aspects :

1) Les hypothèses du programme sont-elles validées?

2) La situation démographique et sanitaire au Bazèga justifie-t-elle la mise en ouvre d'un tel programme de planification familiale?

\subsubsection{Les hypothèses du programme sont-elles validées?}

Les objectifs de l'intervention s'appuient sur un certain nombre d'hypothèses implicites et explicites illustrées dans la Figure 1. Ces hypothèses sont-elles validées ? Etaient-elles pertinentes ? Les réponses à ces questions devraient montrer dans quelle mesure l'intervention a atteint ses objectifs et par quels mécanismes ou dans le cas contraire, quelles sont les raisons de son échec (des explications plus détaillées du cadre d'analyse sont fournies en annexes de ce rapport).

Figure 1 : Les hypothèses de l'intervention DBC-PF au Bazèga

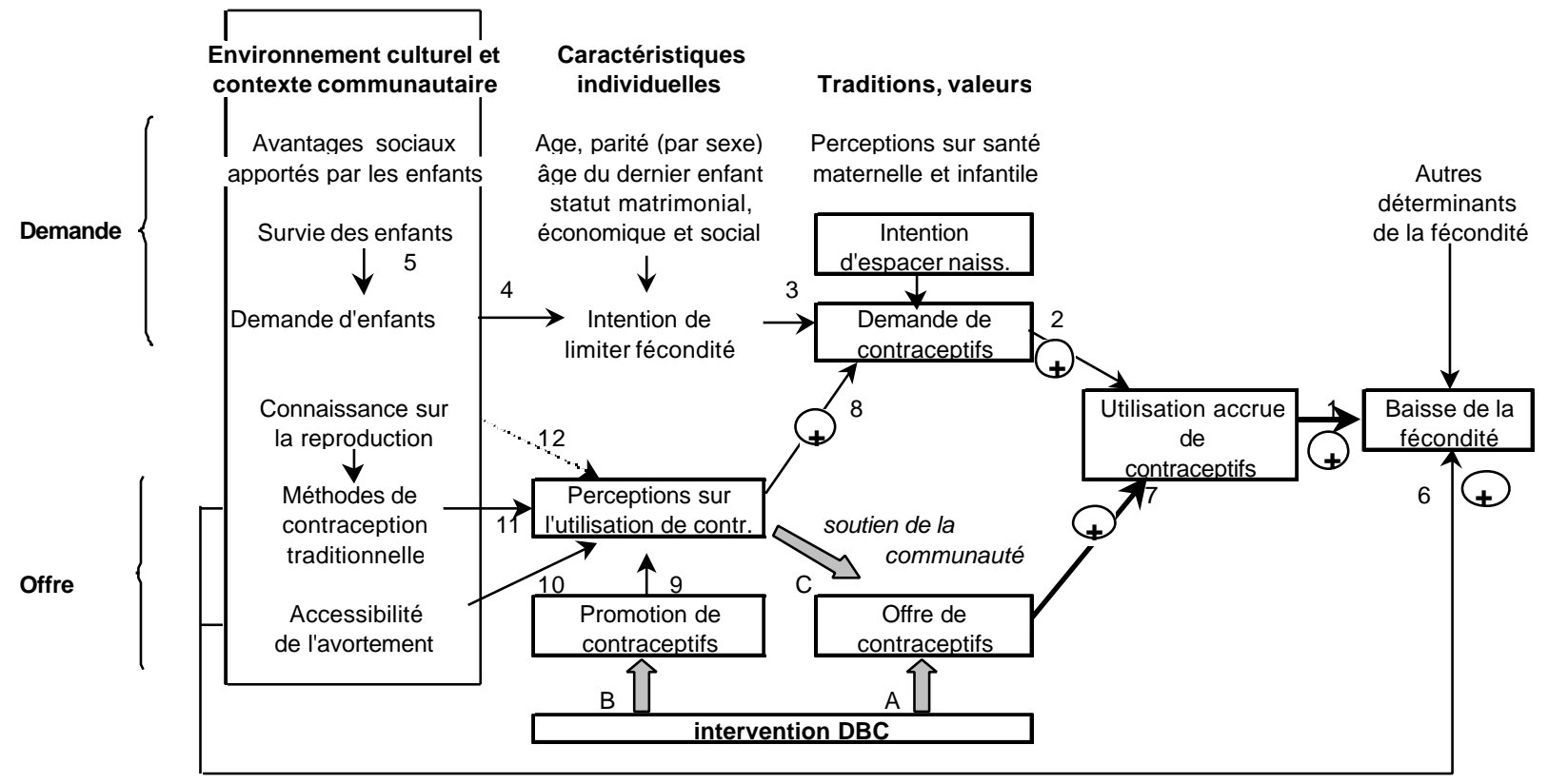

(UERD, 1997) 
Pour faciliter l'évaluation, nous décomposons les hypothèses de l'intervention en plusieurs séries de questions opérationnelles (thèmes). Il s'agit en effet de mettre à jour les liens complexes qui existent entre les différents facteurs qui sont susceptibles d'augmenter l'utilisation de la contraception et de réduire la fécondité.

Pour valider les hypothèses de l'intervention, nous répondrons à quatre questions :

a) Observe-t-on une volonté de maîtriser la fécondité ?

On observe une volonté de maitriser la fécondité lorsqu'une femme déclare avoir l'intention :

- d'espacer les naissances

- de limiter sa descendance

b) La volonté de maîtriser sa fécondité implique-t-elle une demande en méthodes modernes de contraception?

c) Les connaissances, attitudes et pratiques en matières de planification familiale peuvent-elles être modifiées pour accroître la demande en méthodes de contraception modernes?

d) La prévalence de l'utilisation de contraception est-elle faible parce que l'offre est inadéquate ?

\section{a. Quelles sont les pratiques en matière de fécondité observées dans les quatre zones ?}

Nous cherchons ici à analyser les attitudes et pratiques pour établir s'il existait avant l'intervention une demande de services de planification familiale.

$1 \quad$ Les femmes espacent-elles leurs naissances?

La pratique de l'espacement des naissances en Afrique a été amplement documentée. Les rapports qualitatif et quantitatif de base du LSC [UERD, 1997] ont montré que l'intervalle intergénésique «normal » ou souhaité est de plus de deux ans, de préférence trois ans (ces résultats seront repris et analysés plus en détail plus bas). Ainsi, les programmes planification familiale en Afrique sont généralement présentés aux femmes comme des services de santé leur permettant de concrétiser leurs désirs en matière d'espacement de naissances plutôt que de limiter le nombre total de naissances. Or pour avoir un effet, une telle approche doit avoir lieu dans une population ou les femmes connaissent des difficultés à espacer leurs naissances. Ainsi, plus les intervalles intergénésiques au Bazèga avant l'intervention sont courts, plus grand peut être l'effet de l'intervention. A contrario, si on observe que la plupart des femmes réussissent à espacer leurs naissances en l'absence de contraceptifs, il est peu probable qu'une distribution de ceux-ci a un effet sur la durée de l'intervalle intergénésique. 
La majorité des discussions dirigées de groupes ont indiqué qu'une période de trois ans entre un accouchement et la prochaine grossesse était la «norme » pour «que l'enfant ne soit plus collé à sa mère ». D'autres groupes ont même précisé qu'il fallait que l'enfant puisse mettre son cadet au dos, afin que la maman ne souffre pas, ce qui laisse supposer que l'enfant ait suffisamment de force, soit au moins 4 ou 5 ans.

Les naissances rapprochées sont d'ailleurs l'objet de moqueries. En effet, si une femme dont l'enfant ne court pas encore à la rencontre de son père, est à nouveau en grossesse, elle est l'objet de railleries et de désapprobation de l'entourage. Le nouveau-né vient casser la croissance de l'enfant : «kao podege» (rendre infirme).

Le tableau ci-dessous présente les intervalles intergénésiques médians entre les naissances des cinq dernières années (naissance de rang 1 évidemment exclues) et les compare avec les résultats de l'EDS/Burkina Faso. 


\section{Tableau 4 : Intervalle intergénésique.}

Répartition (en \%) des naissances des cinq années précédant l'enquête par nombre de mois écoulés depuis la naissance précédente, selon l'âge de la mère, le rang de naissance de l'enfant et la Zone. Comparaison avec l'EDS/Burkina Faso, Ouagadougou, zones rurales et total.

\begin{tabular}{|c|c|c|c|c|c|c|c|c|}
\hline & \multicolumn{6}{|c|}{ Nombre de mois écoulés depuis la naissance précédente } & \multirow{2}{*}{$\begin{array}{l}\text { Effectifs de } \\
\text { naissances }\end{array}$} & \multirow{2}{*}{$\begin{array}{l}\text { Durée } \\
\text { médiane }\end{array}$} \\
\hline & $7-17$ & $18-23$ & 24-35 & $36-47$ & 48 et plus & Total & & \\
\hline \multicolumn{9}{|l|}{ Classe d'âge } \\
\hline $15-19$ & 33,3 & 0,0 & 50,0 & 16,7 & 0,0 & 100,0 & 6 & 28,4 \\
\hline $20-29$ & 2,3 & 8,0 & 38,9 & 38,2 & 12,6 & 100,0 & 398 & 36,5 \\
\hline 30-39 & 1,3 & 4,1 & 32,6 & 42,3 & 19,8 & 100,0 & 319 & 38,5 \\
\hline $40-49$ & 5,3 & 5,3 & 21,1 & 32,9 & 35,5 & 100,0 & 76 & 42,6 \\
\hline \multicolumn{9}{|l|}{ Rang } \\
\hline $2-3$ & 1,6 & 6,9 & 37,9 & 37,3 & 16,3 & 100,0 & 319 & 36,5 \\
\hline $4-6$ & 1,8 & 5,0 & 33,2 & 42,1 & 17,9 & 100,0 & 340 & 37,6 \\
\hline 7 ou plus & 5,7 & 7,1 & 31,4 & 36,4 & 19,3 & 100,0 & 140 & 36,5 \\
\hline \multicolumn{9}{|l|}{ Zone } \\
\hline A & 1,4 & 5,7 & 34,3 & 37,9 & 20,7 & 100,0 & 140 & 37,5 \\
\hline B & 3,2 & 7,1 & 34,6 & 41,0 & 14,1 & 100,0 & 156 & 36,5 \\
\hline $\mathrm{C}$ & 1,2 & 4,4 & 33,5 & 43,5 & 17,4 & 100,0 & 161 & 37,6 \\
\hline $\mathrm{D}$ & 2,9 & 6,7 & 35,7 & 36,8 & 17,8 & 100,0 & 342 & 36,5 \\
\hline Total LSC 96 & 2,4 & 6,1 & 34,8 & 39,2 & 17,5 & 100,0 & 799 & 37,5 \\
\hline \multicolumn{9}{|l|}{ EDS/BF93 } \\
\hline Ouagadougou & 4,2 & 8,6 & 38,0 & 27,7 & 21,4 & 100,0 & 372 & 34,7 \\
\hline rural & 5,2 & 9,7 & 40,7 & 28,8 & 15,5 & 100,0 & 4502 & 35,6 \\
\hline Total & 5,0 & 9,7 & 40,7 & 28,4 & 16,1 & 100,0 & 5190 & 34,7 \\
\hline
\end{tabular}

Source : LSC (UERD), enquête de base 1996 et EDS/Burkina Faso, 1993

On constate que l'espacement des naissances dans les quatre zones du Bazèga est légèrement plus long que dans la médiane rurale selon les données de l'EDS. Celles-ci montrent que l'intervalle intergénésique à Ouagadougou n'est pas très différent de celui observé dans les zones rurales. Partout, l'intervalle intergénésique varie en fonction de l'âge de la mère : plus celle-ci est âgée, plus l'intervalle est long. En revanche, le rang de naissance n'a pas d'influence sur la durée de l'intervalle.

La majorité des femmes du Bazèga, comme partout ailleurs au Burkina, continue d'espacer leurs naissances selon les normes traditionnelles, et ce en l'absence de contraception moderne. Notons toutefois qu'il s'agit de l'intervalle entre deux naissances vivantes : on ne tient pas compte des grossesses improductives et des mortnés. 
Selon $86 \%$ des femmes et $84 \%$ des hommes, une femme qui vient d'accoucher doit attendre au moins 3 ans avant d'avoir un autre enfant, résultat confirmé par l'enquête qualitative. On constate un complet accord entre les hommes et les femmes (cf. Figure 2). En revanche, le sens donné au concept d'espacement des naissances varie selon le sexe de l'enquêté. Pour les femmes, espacer les naissances est important pour la santé de la mère : elle peut se reposer, elle vieillit moins vite ( $42 \%$ des réponses $\left.{ }^{7}\right)$. La santé de l'enfant (le bébé se développer) arrive en deuxième position avec $24 \%$ des réponses. Pour les hommes, l'espacement des naissances permet d'abord à la mère de mieux s'occuper des enfants (32\%). La santé de la mère arrive en deuxième position avec $24 \%$ des réponses. L'argument économique pour avoir moins d'enfants est plus rarement évoqué, et l'est plutôt par les hommes.

\section{Figure 2 : Concept et durée d'espacement des naissances, selon le sexe.}

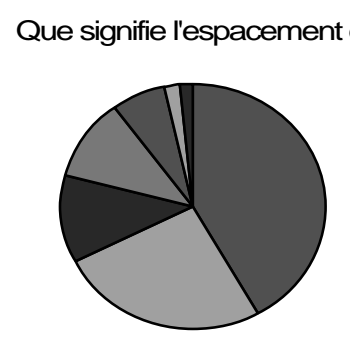

Femmes

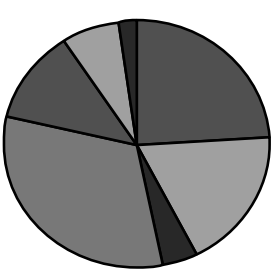

Hommes

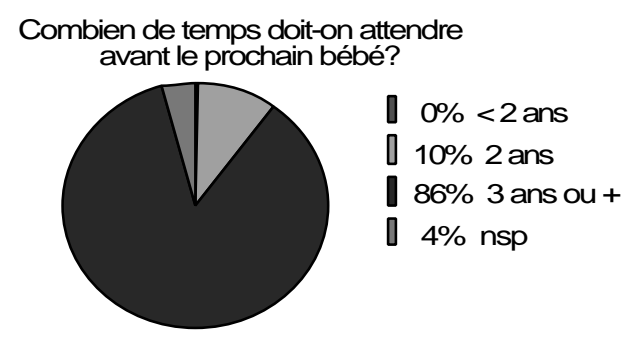

Femmes

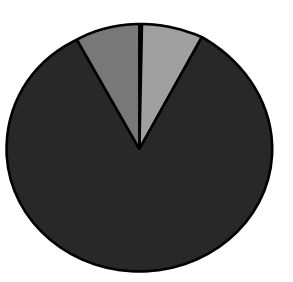

$0 \%<2$ ans

[. $7 \% 2$ ans

$84 \% 3$ ans ou +

[ $8 \% \mathrm{nsp}$

$12 \%$ moins d'enfants

$7 \%$ économiser

| $2 \%$ autre

Hommes

L'espacement des naissances observé est donc conforme aux valeurs exprimées par les hommes comme par les femmes. La question qui reste à élucider est comment les couples atteignent leurs objectifs : abstinence post partum ? Pratiques contraceptives ? Allaitement prolongé ? Avortement? 
Une femme peut souhaiter limiter sa fécondité (c'est à dire ne plus avoir d'enfant) pour plusieurs raisons : elle estime qu'elle est trop âgée ; elle a déjà une fille qui a accouché ou qui est enceinte (cf. entretiens qualitatifs); elle a atteint le nombre désiré d'enfants. Ces trois raisons sont toutes fortement liées à l'âge. Nous abordons donc cette question sous plusieurs angles : celui du nombre idéal d'enfants ; la comparaison de celui-ci avec le nombre d'enfants survivants ; les attitudes et pratiques de femmes qui ont atteint le nombre idéal de filles et de garçons.

La Figure 3, graphique de gauche, montre la relation entre l'âge de la femme et son désir de ne plus avoir d'enfants. Les femmes qui souhaitent ne plus avoir d'enfant à moins de 30 ans sont rares. Peu de femmes (moins de 1\%) disent que «ça dépend du partenaire ». Les femmes qui s'en remettent à Dieu (6\%) désirent probablement d'autres enfants, et par leur attitude ne sont sans doute pas candidates à l'utilisation d'une méthode contraceptive.

\section{Figure 3 : Désir d'enfant supplémentaire selon l'âge et le nombre d'enfants survivants.}
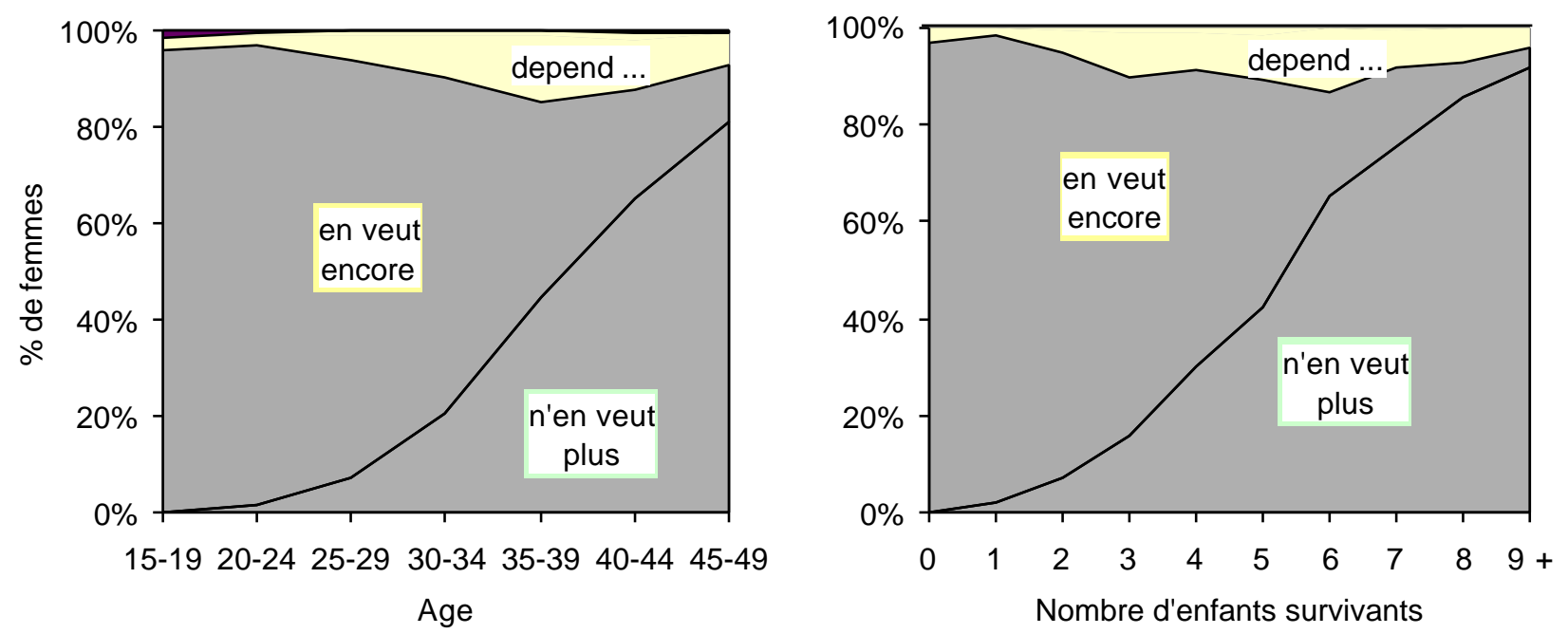

Source : LSC (UERD), enquête de base 1996

Le nombre d'enfant en vie influe évidemment aussi sur le désir d'enfant (Figure 3, graphique de droite) : ce n'est qu'à partir du cinquième enfant vivant que la majorité des femmes déclarent ne plus en vouloir, mais cela est dû autant à l'âge de la femme qu'au nombre de ses enfants. En effet, les femmes qui disent ne plus vouloir d'enfant ont en moyenne 39,5 ans, elles ont eu 7,1 naissances vivantes pour 5,4 enfants encore en vie.

\footnotetext{
${ }^{7}$ Plusieurs réponses a cette question étaient possibles.
} 
Il est inconvenant pour une femme d'avoir un enfant lorsqu'elle a un petit-fils. Elle est censée arrêter les relations sexuelles avec son mari lorsque sa belle-fille, femme de son premier fils accouche. Elle change de position et devient grand-mère. Le premier fils en milieu rural demeure dans la concession de son père après son mariage. C'est sa mère qui donne les premiers soins au petit-fils et qui porte le bébé au dos. Il ne faut donc pas qu'il y ait concurrence avec un de ses propres enfants

On peut dès à présent signaler que l'impact d'une distribution, communautaire ou non, de contraceptifs aura peu d'impact sur la fécondité, ces femmes étant pour la plupart proche de la fin de leur vie reproductive. D'ailleurs, il est probable que certaines d'entre elles disent ne plus vouloir d'enfant sachant qu'elles ont peu de chance de tomber enceintes. Peu seront donc candidates à la planification familiale, et celles qui le seraient risquent de mobiliser inutilement des ressources du programme qui seraient mieux investies ailleurs (chez les jeunes filles notamment, qui sont plus exposées au risque de grossesse et qui ont plus de difficulté à espacer leurs naissances (cf. Tableau 4))

Ce nombre moyen d'enfants en vie des femmes ne souhaitant plus avoir d'enfants $(5,4)$ est cohérent avec les données présentées dans le Tableau 5. Les femmes déclarent comme idéal au moins trois garçons (3,2 en moyenne) et entre deux et trois filles $(2,5)$, soit au total 5,7 enfants.

Tableau 5 : Nombre idéal de garçons et de filles.

Enquête de base (UERD-1996)

Zone

A $\quad$ B $\quad$ C $\quad$ D $\quad$ Total
EDS-Burkina Faso (1993)

Lieu de résidence

rural Ouaga. Burkina

\section{Nombre idéal moyen d'enfants}

\section{femmes:}

$\begin{array}{lllllllll}\text { garçons } & 3,1 & 3,2 & 3,3 & 3,0 & 3,2 & & & \\ \text { filles } & 2,4 & 2,6 & 2,6 & 2,4 & 2,5 & & & \\ \text { enfants } & 5,5 & 5,8 & 6,0 & 5,4 & 5,7 & 6,2 & 4,1 & 5,7\end{array}$

\section{hommes}

\begin{tabular}{lllllllll} 
garçons & 4,3 & 4,6 & 4,5 & 4,0 & 4,3 & & & \\
filles & 3,1 & 3,7 & 3,4 & 3,0 & 3,3 & & & \\
enfants & 7,4 & 8,3 & 7,9 & 6,9 & 7,7 & 8 & 4,2 & 7,1 \\
\hline
\end{tabular}


Source : LSC (UERD), enquête de base 1996 et EDS/Burkina Faso, 1993

Comparons maintenant le nombre idéal d'enfants ${ }^{8}$ avec l'expérience des femmes qui arrivent à la fin de leur vie reproductive (Tableau 6). On constate d'abord que les femmes de 45-49 ans ont perdu en moyenne deux enfants chacune. Ainsi, elles ont atteint la taille idéale moyenne en compensant la mortalité par deux naissances vivantes supplémentaires. Si les femmes du Bazèga tiennent compte de l'expérience de leurs aînées, elles seront bien tentées d'avoir elles aussi sept enfants pour atteindre le nombre idéal de cinq-six enfants, sauf si elles anticipent une très forte baisse de la mortalité infantile et juvénile, ce qui est peu probable vu le niveau encore très élevé de mortalité infantile et juvénile dans le Bazèga (cf. page 65)

Tableau 6 : Descendance finale et nombre d'enfants survivants, selon le sexe.

\begin{tabular}{|c|c|c|c|c|c|c|c|c|}
\hline & \multicolumn{5}{|c|}{ Enquête de base (UERD-1996) } & \multicolumn{3}{|c|}{ EDS-Burkina Faso (1993) } \\
\hline & \multirow[b]{2}{*}{ A } & \multicolumn{2}{|c|}{ Zone } & \multirow[b]{2}{*}{$\mathrm{D}$} & \multirow[b]{2}{*}{ Total } & \multicolumn{3}{|c|}{ Lieu de résidence } \\
\hline & & $\mathrm{B}$ & $\mathrm{C}$ & & & rural & Ouaga. & Burkina \\
\hline \multicolumn{9}{|l|}{ Femmes: nombre moyen de } \\
\hline naissances vivantes (40-49 ans) & 7,1 & 7,4 & 7,2 & 7,2 & 7,3 & 7,5 & 6,8 & 7,4 \\
\hline naissances vivantes (45-49 ans) & 7,4 & 8,0 & 7,2 & 7,2 & 7,4 & & & 7,7 \\
\hline enfants en vie (45-49 ans) & 5,1 & 6,2 & 4,9 & 5,2 & 5,3 & & & 5,4 \\
\hline \multicolumn{9}{|l|}{ Hommes: nombre moyen de } \\
\hline naissances vivantes (>50 ans) & 11,6 & 12,6 & 11,3 & 11 & 11,6 & & & \\
\hline naissances vivantes (>60 ans) & 12,8 & 12,3 & 11,2 & 12,3 & 12,1 & & & \\
\hline enfants en vie (>60 ans) & 8,4 & 9,5 & 8 & 8,3 & 8,6 & & & \\
\hline
\end{tabular}

Source : LSC (UERD), enquête de base 1996 et EDS/Burkina Faso, 1993

Le nombre idéal d'enfants des hommes (en fait des chefs de ménage) est fortement supérieur, en moyenne, à celui des femmes. Cet écart n'indique forcément de désaccord entre hommes et femmes puisque la polygamie permet aux premiers d'atteindre leur objectif de fécondité sans contraindre leurs épouses à dépasser le leur. Or 38\% des hommes enquêtés sont polygames. Il est aussi important de noter que la descendance finale des hommes de plus de 60 ans dépasse le nombre idéal moyen d'enfants déclaré par

\footnotetext{
${ }^{8}$ Rappelons ici les biais inhérents à cet indicateur : (1) les femmes peuvent «justifier » une fécondité mal maîtrisée en déclarant a posteriori un nombre idéal d'enfant supérieur à celui qu'elles auraient réellement souhaité ; (2) les femmes qui donnent des réponses non numériques («cela dépend de Dieu ») sont exclues de calculs, ce qui fait baisser la moyenne car ces femmes désirent généralement plus d'enfants que celles qui donnent une réponse précise ; (3) pour certaines femmes le nombre d'enfants idéal dépend de sa situation au moment de l'enquête, alors que pour d'autres il s'agit du nombre d'enfants désirés dans de conditions idéales [Bongaarts, 1990,Pritchett, 1994].
} 
l'ensemble des hommes. Ceci indique probablement une baisse de la pression pronataliste exercée par les hommes suite a la dégradation des conditions de vie et l'érosion du pouvoir de la gérontocratie lignagère.

Pour conclure cette question, on peut dire que les femmes n'hésitent pas à déclarer qu'elles ne souhaitent plus avoir d'enfant, mais ce souhait ne concerne pratiquement que les femmes qui ont plus de cinq enfants et/ou qui ont plus de 35 ans, ce qui limite l'effet de l'intervention sur les indicateurs de fécondité.

$4 \quad$ Une femme (un homme) peut-elle (il) convaincre son (sa) conjoint(e) de réguler sa fécondité?

Les résultats de l'enquête de base indiquent que les hommes et les femmes ne discutent pas avec leurs conjoints des problèmes de fécondité. Cette absence de communication ne constitue pas nécessairement un obstacle à une régulation efficace de la fécondité, sauf si les femmes qui souhaitent réguler leur fécondité ne peuvent outrepasser, ouvertement ou non, la résistance de leur conjoint.

La situation est la même à Navrongo où le rôle dominant des hommes limite le dialogue au sein du couple. En l'absence de dialogue, les préférences des maris ont peu d'effet sur les choix contraceptifs de leurs épouses. En revanche, les couples qui dialoguent ont quatre fois plus de chance d'utiliser une méthode contraceptive [Phillips et al., 1997].

Les femmes ont une variété de stratégies qu'elles pensent pouvoir mettre en œuvre pour convaincre leur mari de planifier leur famille.

- par le dialogue : par les échanges; par la causerie, en discutant; mais aussi par des flatteries, avec douceur et gentillesse; en lui faisant des cadeaux parfois en négociant dur. Ces femmes font confiance à l'entente entre époux, à la compréhension du conjoint. Certaines font aussi confiance à la force de leurs arguments: dans mes explications, je saurai le convaincre D'autres en revanche font appel a la propagande officielle : je vais lui faire écouter les conseils sur la PF à la radio ; nous allons discuter sur les bases de la PF.

- en mettant l'accent sur les avantages économiques d'une famille restreinte : en lui montrant que la vie est dure; je vais lui expliquer qu'il ne pleut pas et qu'il n'y a pas à manger; je vais lui faire comprendre les difficultés de la vie actuelle; que la vie est dure; les enfants sont nombreux et la vie est dure; je lui rappellerai qu'il n'y a rien à manger et les pluies sont insuffisantes;

Elles pensent aussi qu'il sera sensible à l'idée qu'il faut qu'on espace pour pouvoir s'occuper des enfants; que les enfants sont difficiles à élever. 
- en mettant l'accent sur la santé de la mère : je suis maladive et il le sait; [je vais] lui parler de ma santé; je suis fatigué pour faire des enfants; lui-même va trouver que je suis fatigué; il faut aussi que la mère se repose; je lui dirai que je suis fatigué.

ou de la santé de l'enfant : je lui dirai de laisser l'enfant se développer sinon il va mourir; l'excès d'accouchements peut entrainer la mort; si l'enfant n'est pas développé, il devra attendre.

Pour convaincre leur mari, certaines femmes se propose d'amener leur partenaire à la FS : nous pouvons aller voir les infirmière pour le convaincre; on ira au dispensaire pour qu'on lui explique mieux.

D'autre pensent qu'il est déjà sensibilisé. Il s'agit ici pour la plupart de couples qui ont déja discuté sur ce point et qui sont arrivé à un accord: nous sommes tous les deux pour l'espacement des naissances; nous nous comprenons très bien; c'est pour le bien de a famille ;mon mari a été sensibilisé à ce sujet à une réunion sur la PF; on s'est déjà entendu que je ne ferai plus d'enfants; mon mari ne veut plus d'enfants.

A l'opposé, il y les couples ou l'homme impose sa volonté : je suis le maitre; j'impose ma décision; c'est moi le chef; je m'impose; elle doit accepter; si elle refuse, je la répudie; il n'y a pas deux capitaines dans un bateau; c'est l'homme qui décide; la femme n'a pas le droit de refuser; je divorce.

D'autres stratégies sont plus extrêmes : en refusant de faire des rapports sexuels; en fuyant chez mes parents; ou encore : en utilisant une méthode à l'insu du mari.

Il n'y a pas que l'époux qui est susceptible d'empêcher sa femme de réduire sa fécondité. Tout au long des entretiens de groupes sur la procréation, le lignage et sa pérennité apparaissent au centre des préoccupations des aînés. Les enfants représentent une force de travail, et, lorsqu'ils sont nombreux, une source de prestige et de fierté pour le lignage. Si la contraception permet aux femmes de limiter le nombre de ses enfants, elle ne l'isole pas pour autant du tissu de relations sociales. Or celui-ci, fortement pro-nataliste, est très contraignant. Les règles de conformité très prégnantes, les sanctions sévères. Les femmes innovatrices doivent développer des stratégies spécifiques pour affronter la volonté du lignage. La promotion de la contraception ne peut ignorer l'étroitesse de la marge de liberté de la femme dans une société où les intérêts du lignage priment sur ceux des individus. Elle doit vider à rendre son usage plus légitime afin de faciliter les échanges entre époux sur le sujet.

$5 \quad$ La composition par sexe de la descendance atteinte influence-t-elle le désir d'enfants ?

La Figure 4 montre la probabilité pour une femme de déclarer vouloir un enfant supplémentaire (axe vertical) en fonction du nombre de filles survivantes (axe horizontal dans le graphique de gauche) ou du nombre de 
garçons survivants (axe horizontal dans le graphique de droite). Par exemple, une femme qui deux fils et aucune fille a une probabilité de 0,9 de déclarer vouloir un enfant supplémentaire. Cette probabilité passe à 0.8 si elle a une fille, et à 0,6 si elle en a deux. On remarque, en comparant les lignes du haut sur les deux graphiques que la demande d'enfant est aussi forte lorsque l'on n'a pas de fille que lorsqu'on n'a pas de garçon. La demande d'enfant pour les femmes sans garçons est forte quel que soit le nombre de filles qu'elles ont (graphique de gauche). La demande d'enfant chez les femmes sans filles est tout aussi forte mais décline fortement à partir du troisième garçon (graphique de droite). En d'autres termes, ce n'est que lorsqu'une femme a déjà quatre garçons en vie que la probabilité de ne plus vouloir d'enfant approche 0,5 (contre 0,9 pour une femme qui a trois garçons).

Ces graphiques montrent bien que les hommes et les femmes désirent un minimum d'enfants de chaque sexe plutôt qu'un nombre maximum d'enfants. Il est sans doute difficile à une femme de se résoudre à avoir moins de 3 garçons et moins de deux filles, ce qui est cohérent avec ce qu'elles déclarent être le nombre idéal d'enfants (cf. Tableau 5). Dans un contexte de mortalité élevée et dans l'impossibilité de choisir le sexe de ses enfants, le désir d'avoir suffisamment d'enfants de chaque sexe plutôt qu'un nombre maximum d'enfants contribue à maintenir la fécondité à un niveau élevé.

Figure 4 : Probabilité pour une femme de vouloir un autre enfant, selon le nombre de garçons et de filles survivants.
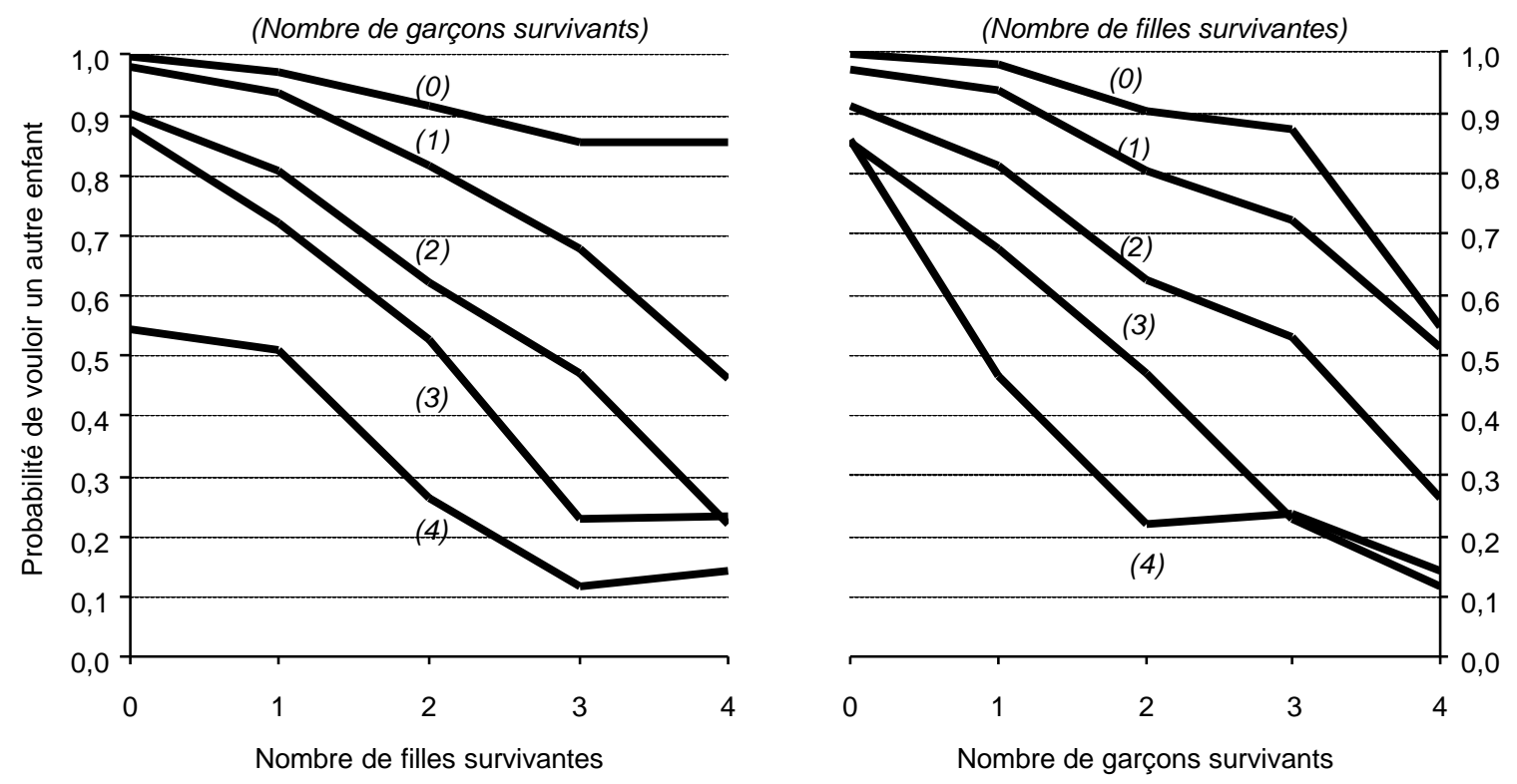

Source : LSC (UERD), enquête de base 1996 
6 Les couples connaissent-ils la manière de maîtriser leur fécondité de façon naturelle ?

Les méthodes naturelles les plus citées par les femmes comme les hommes sont l'abstinence (périodique ou post-partum) et le retrait. Selon nos premières investigations qualitatives, le cycle de la conception n'est pas clairement perçu. Il n'y a pas de mot en Moore pour désigner la conception. L'eau de l'homme (le sperme) ensemence la graine et lui permet de grandir. Si les femmes connaissent la durée d'un cycle, beaucoup semblent ignorer quand elles sont fécondes. Des femmes affirment qu'elles peuvent tomber enceinte pendant ses règles. Les règles (pekre) sont intimement liées au cycle lunaire. A certaines stades de la formation de la lune, les relations sexuelles sont interdites sous peine d'avoir un enfant idiot. Des entretiens semi-directifs nous permettrons de mieux analyser les connaissances des femmes de la biologie de la reproduction afin de formuler des recommandations relatives à la promotion de la contraception.

$7 \quad$ Les couples comprennent-ils comment fonctionnent les méthodes contraceptives modernes ?

A peine la moitié des femmes en union ont entendu parlé d'une méthode moderne de contraception (Tableau 7). La plus connue est le préservatif, sans doute grâce aux messages sur le sida et les MST. Moins du tiers des femmes connaissent la pilule, une proportion inférieure à celles qui connaissent les injections. Ces chiffres sont comparables à ceux de l'EDS pour les zones rurales, mais très inférieurs à ceux de Ouagadougou $(96,8 \%)$ qui pourtant n'est pas loin du Bazèga.

La proportion de femmes ayant déjà utilisé une de ces méthodes est bien entendu encore plus faible, entre 4 et $12 \%$ selon les zones. La plus forte proportion de femmes ayant utilisé la pilule (11\%) pourrait être le résultat du programme de planification familiale de Save the Children. 
Tableau 7 : Connaissances et pratiques de la contraception moderne.

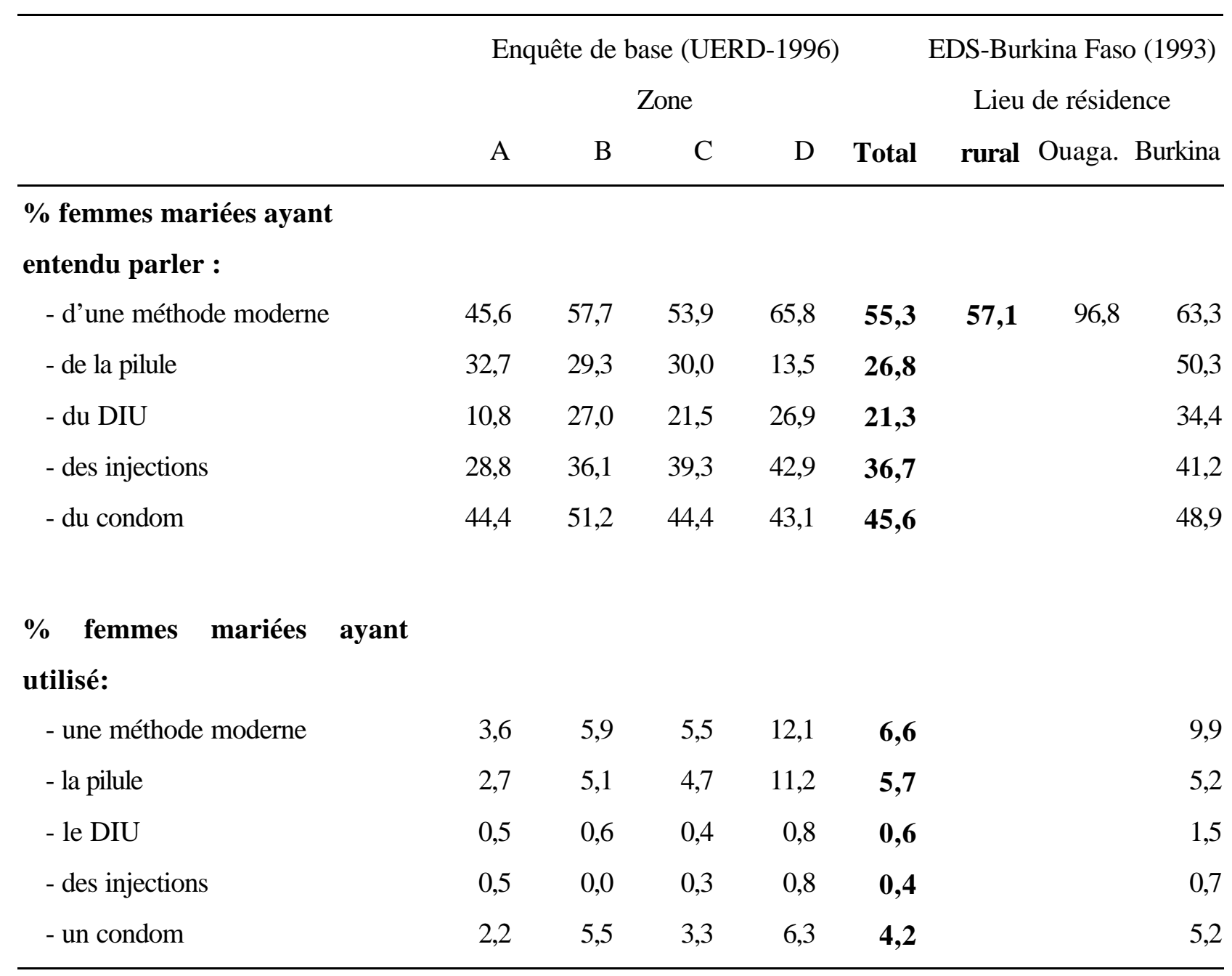

Source : LSC (UERD), enquête de base 1996 et EDS/Burkina Faso, 1993

Ces résultats montrent que la connaissance des femmes sur les méthodes contraceptives modernes n'est pas suffisamment répandue. De plus, ce n'est pas parce qu'une femme a entendu parler d'une méthode qu'elle sait comment celle-ci fonctionne. Il est même fort probable que les femmes qui ont utilisé une méthode moderne dans le passé n'aient qu'une idée confuse de son fonctionnement.

8 La coutume d'abstinence post-partum est-elle toujours observée ? Quelle est sa durée ?

Les résultats qualitatifs de l'enquête de base indiquent que la pratique de l'abstinence post partum est moins respectée que par la passé. Ceci a aussi été documenté ailleurs en Afrique de l'Ouest [Bledsoe et al., 1994]. Il est plus rare que les femmes vivent longtemps séparée de leurs époux suite à une naissance. Les méthodes modernes de contraceptions pourraient donc intéresser les couples qui souhaite réduire la durée d'abstinence tout en respectant les normes d'espacement des naissances sans recourir à l'avortement. Ceci a été observé 
à Navrongo [Biddlecom et al., ], où on explique une partie de la discontinuité de l'utilisation de la pilule par la sortie des couples de la période d'abstinence imposée par la coutume.

\section{$9 \quad$ L'intervalle moyen entre deux naissances est-il stable au Bazèga ?}

Lors des enquêtes qualitatives sur les déterminants de la fécondité, des participants affirmaient qu'il y avait plus de naissances rapprochées de nos jours car les couples respecteraient moins que par le passé la coutume de l'abstinence post partum ( 3 ans). Ces personnes attribuaient cette évolution à un relâchement des mœurs et au nouveau style de vie familiale (selon laquelle mari et femmes dorment sous le même toit pendant la période d'abstinence post partum). Or si on compare les intervalles intergénésiques par âge entre 1988 et 1997 avec ceux de la période 1978-87, on constate plutôt un léger rallongement de la durée médiane entre deux naissances (Tableau 8). Même s'il est possible que ce rallongement soit dû aux effets de mémoire (plutôt qu'à une utilisation accrue de méthodes contraceptives), il est peu probable que les pratiques en matière d'espacement des naissances ont évolué de façon substantielle dans le Bazèga ces 20 dernières années. Il est possible que comme les femmes plus jeunes espacent moins leurs naissances, les participants en déduisent un relâchement des mœurs. Or comme le montre le Tableau 8, il ne s'agit là d'un effet d'âge et non d'un effet de génération. 


\section{Tableau 8 : Evolution de l'intervalle intergénésique.}

Nombre médian de mois écoulés depuis la naissance précédente

\begin{tabular}{lccr}
\hline & $78-87$ & $88-97$ & Ecart \\
\hline Classe d'âge & & & \\
$15-19$ & 27,4 & 28,4 & 1,0 \\
$20-29$ & 33,0 & 35,5 & 2,5 \\
$30-39$ & 34,5 & 37,5 & 3,0 \\
$40-49$ & - & 41,6 & - \\
Rang & & & \\
$2-3$ & 34,5 & 36,5 & 2,0 \\
$4-6$ & 33,5 & 37,5 & 4,0 \\
7 ou plus & 30,4 & 36,5 & 6,1 \\
Zone & & & \\
A & 34,0 & 37,5 & 3,5 \\
B & 34,5 & 36,5 & 2,0 \\
C & 33,5 & 37,5 & 4,0 \\
D & 33,4 & 36,5 & 3,1 \\
Total LSC 96 & 33,5 & 36,5 & 3,0 \\
Source : LSC (UERD), enquête de base 1996 et EDS/Burkina Faso, 1993
\end{tabular}

\section{b. Quel est le niveau de la demande de contraception au Bazèga ?}

1 Que connaissent les couples (hommes et femmes) au sujet de la contraception (méthodes, effets secondaires, sources d'information et d'approvisionnement) ?

Quand le degré de connaissance sur la contraception sera établi au niveau villageois, on cherchera à savoir s'il est associé au niveau d'éducation afin de voir si le faible niveau d'éducation observé dans l'enquête de base est un obstacle à l'utilisation de la contraception.

$2 \quad$ Y a t-il une demande de contraception?

Commençons par distinguer demande de besoins en matière de planification familiale.

Les enquêtes démographiques de santé (EDS) définissent la demande potentielle totale en PF comme la somme des besoins satisfaits et des besoins non satisfaits pour espacer ou limiter les naissances. Elle s'exprime en pourcentage des femmes mariées.

Les besoins non satisfaits pour espacer les naissances concernent les femmes enceintes ou en aménorrhée dont la dernière grossesse ne s'est pas produite au moment voulu, ou, pour les femmes qui ne sont ni 
enceintes, ni en aménorrhée qui ont déclarer vouloir attendre avant leur prochaine naissance et qui n'utilise pas de méthode contraceptive.

Les besoins non satisfaits pour limiter les naissances concernent les femmes enceintes ou en aménorrhée dont la dernière grossesse n'était pas désirée, ou, pour les femmes qui ne sont ni enceintes, ni en aménorrhée, qui déclarent ne plus vouloir d'enfants et qui n’utilise pas de méthode contraceptive. Les femmes qui déclarent ne pas pouvoir avoir d'enfant (stérilité, mais surtout ménopause) sont exclues des calculs.

Tableau 9 : Besoins en planification familiale en 1996 (en \%).

\begin{tabular}{|c|c|c|c|c|c|c|c|c|}
\hline & \multicolumn{5}{|c|}{ Enquête de base (UERD-1996) } & \multicolumn{3}{|c|}{ EDS-Burkina Faso (1993) } \\
\hline & \multicolumn{4}{|c|}{ Zone } & \multirow[b]{2}{*}{ Total } & \multicolumn{3}{|c|}{ Lieu de résidence } \\
\hline & A & $\mathrm{B}$ & $\mathrm{C}$ & $\mathrm{D}$ & & rural & Ouaga. & Burkina \\
\hline \multicolumn{9}{|l|}{ Besoins non satisfaits en PF : } \\
\hline - pour espacer les naissances & 28,8 & 35,9 & 29,4 & 27,9 & 30,4 & 20,2 & 19,0 & 20,3 \\
\hline - pour limiter les naissances & 10,5 & 11,9 & 11,1 & 13,9 & 11,7 & 8,3 & 11,5 & 8,8 \\
\hline - total & 39,3 & 47,8 & 40,5 & 41,7 & 42,1 & 28,5 & 30,5 & 29,1 \\
\hline \multicolumn{9}{|l|}{ Besoins en PF satisfaits: } \\
\hline Prévalence méthodes modernes & 3,7 & 3,6 & 3,2 & 7,4 & 4,8 & 4,2 & 30,6 & 7,9 \\
\hline Demande potentielle totale de PF & 43,1 & 51,4 & 43,7 & 49,1 & 46,9 & 32,7 & 61,1 & 37,0 \\
\hline Demande satisfaite / dem. totale & 8,7 & 7,0 & 7,3 & 15,1 & 10,1 & 12,7 & 50,1 & 21,4 \\
\hline
\end{tabular}

Source : LSC (UERD), enquête de base 1996 et EDS/Burkina Faso, 1993

La Figure 5 présente les besoins en matière de PF (espacer, limiter les naissances ou rien) selon l'âge de la femme. On constate qu'à partir de 25 ans, les besoins sont constants aux environs de 40\%. Ce qui change avec l'âge, c'est la nature du besoin : pour les plus jeunes, c'est d'espacer les naissances, pour les plus âgées, c'est d'éviter d'avoir d'autres enfants. Cela ne signifie pas que les femmes de moins de 25 ans n'ont pas objectivement besoin de PF. Bien au contraire, on a pu constater plus haut (Tableau 4) qu'elles avaient plus de mal à respecter l'intervalle intergénésique de 3 ans. 
Figure 5 : Besoins des femmes en matière de planification familiale selon leur âge.

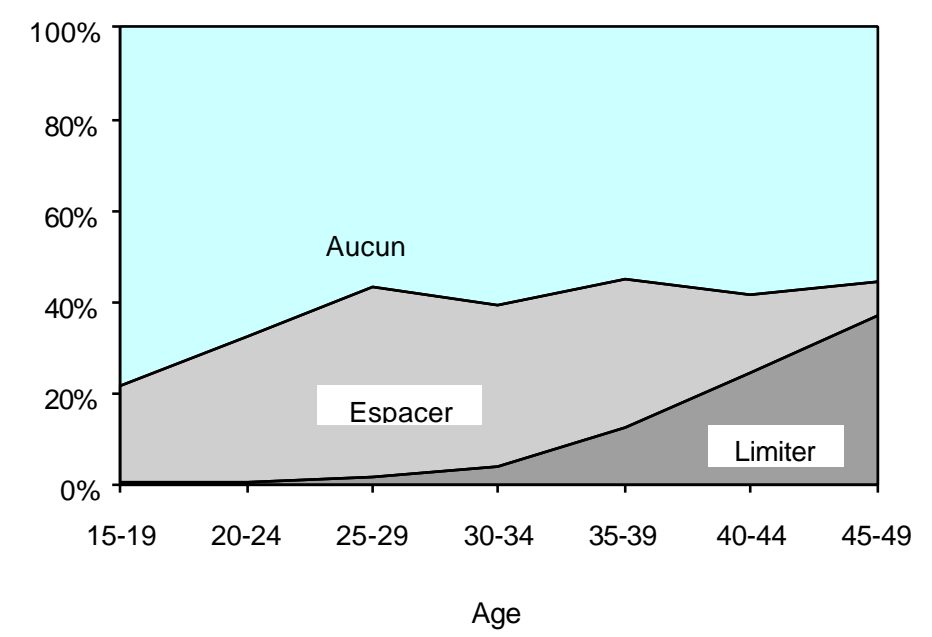

Source : LSC (UERD), enquête de base 1996.

La demande selon l'EDS est la somme des besoins satisfaits et non satisfaits. Ce n'est donc pas une demande exprimée mais seulement une proportion de femmes qui pourraient, si elles en avaient la connaissance et la volonté, être intéressée par la planification familiale. La nuance est de taille.

Nous verrons plus bas (Figure 9 page 59) que plus de $80 \%$ des femmes qui déclarent pratiquer la planification familiale le font en s'abstenant (essentiellement par la traditionnelle abstinence post-partum). Selon l'EDS, les femmes respectant la règle de l'abstinence post-partum sont incluses dans la demande non satisfaite de PF, alors qu'elles ne sont pas exposées au risque de grossesse ${ }^{9}$. La définition de la demande non satisfaite de l'EDS surestime la demande de contraceptifs ( [Pritchett, 1994]).

Il existe aussi un besoin de contraception chez les femmes non mariées, notamment des adolescentes. On sait que celles-ci sont elles aussi exposées aux risque de grossesse et sont souvent acculées à l'avortement clandestin. Toutefois, comme il est plus difficile pour une adolescente célibataire de dire qu'elle a des relations sexuelles, une enquête transversale ne peut que sous-estimer leur besoins en contraceptifs. L'intervention devra donc cibler les adolescentes et les approcher avec des activités spécifiques, avec, comme le proposait FOCUS, des DBC du même âge.

\footnotetext{
${ }^{9}$ Cette remarque doit toutefois être nuancée. Une femme qui s'abstient n'est pas à l'abri des exigences de son mari. On peut donc estimer qu'une femme féconde qui dit s'abstenir pour éviter de tomber enceinte est quand même exposée au risque de grossesse, même si ce risque est moins grand que pour les femmes qui ont repris les rapports sexuels.
} 
On notera enfin que même dans le cas d'une faible demande de contraceptif, la presence de services de planification familiale permet de réduire le nombre de grossesse indésirées car la probabilité de tomber enceinte chez les femmes sexuellement active et ne pratiquant aucune forme de contraception est élevé, de l'ordre de $20 \%$ par mois ( [Bongaarts, 1991])

$3 \quad$ Y a-t-il une demande insatisfaite de méthodes contraceptives modernes ?

def1 : Le niveau de demande insatisfaite de contraception est la proportion des femmes qui n'utilisent pas une méthode alors qu'elle déclare vouloir en utiliser une. On précisera les raisons de l'existence d'une demande insatisfaite.

def2 : Le niveau de la demande de contraception est la proportion de femmes qui déclare vouloir utiliser une méthode donnée si elle était accessible et gratuite.

$4 \quad$ Pour quels types de contraceptifs y a-t-il une demande?

$5 \quad$ Y a-t-il une demande pour les méthodes contraceptives traditionnelles ?

L'abstinence est la seule méthode contraceptive mentionnée par nos informants.

$6 \quad$ Y a-t-il une demande pour l'avortement?

Les résultats d'une enquête exploratoire suggèrent que l'avortement soit une pratique courante au Bazèga. L'avortement pourrait de ce fait constituer une méthode concurrente à la planification familiale. Par exemple, il est possible que des couples aient recours à l'avortement en cas de grossesse trop rapprochée. Cela pourrait en partie expliquer qu'en dépit de changements de mœurs l'intervalle intergénésique est resté conforme à la norme. Dans l'enquête de passage (1998), entre une femme sur cinq et une femme sur quatre déclare connaître une femme de son entourage qui a avorter.

$7 \quad$ La pilule est-elle une alternative à l'avortement ?

La distinction entre contraception et avortement n'est pas forcément évidente ou pertinente chez les villageois. On peut se demander si les connaissances des femmes en la biologie de la reproduction soit suffisante pour leur permettre de faire une telle distinction. Il n'existe pas de terme en Moore pour designer la fécondation, conception, ni a fortiori la contraception.

Selon des agents DBC, certaines femmes leur demande la pilule afin d'avorter (c'est d'ailleurs ainsi que certains vendeurs de médicaments au marché présentent la pilule). Comme par ailleurs la pilule «rentre dans le sang pour empêcher qu'il y ait un bébé » (explication d'un responsable de formation sanitaire) et que, par 
ailleurs, «le sang ne se lave pas » (ziim pa pekd), il n'est pas étonnant que certaines femmes pensent que la pilule rend stérile.

\section{c. Les CAP en matière de contraception peuvent-elles être modifiées afin d'accroître la demande de contraception ?}

Lors de la précédente série de questions, les intentions de maîtriser la fécondité sont analysées. Parmi les personnes enquêtées qui déclarent souhaiter maîtriser leur fécondité, quelques-unes affirment qu'elles n'ont pas l'intention d'utiliser une méthode contraceptive moderne. Dans cette section, nous analysons les raisons pour lesquelles les personnes qui souhaitent maitriser leur fécondité ne veulent pas utiliser la contraception (ou n'en ont pas l'intention). Lorsque ces motifs seront clairement identifiés, ils seront discutés afin de recommander les voies et moyens de promouvoir la PF de manière plus efficace.

1 Quels sont les obstacles à l'utilisation de la contraception moderne parmi les femmes qui désirent maîtriser leur fécondité ?

Cette question d'ordre général nécessite d'être posée lors d'entretiens semi-dirigés et d'entretiens dirigés de groupe. Elle doit se concentrer sur la pression sociale (de la communauté, de la famille, du conjoint), l'acceptabilité culturelle, la confidentialité et l'accessibilité des méthodes. Toutefois, l'enquête de base offre déjà quelques éléments de réponses.

Parmi les femmes qui ne sont pas enceintes, près de $40 \%$ ne prennent pas de contraceptif car elles estiment qu'elles ne sont pas exposées au risque de grossesse : elles ne sont pas mariées, elles n'ont pas de rapports sexuels, ou alors peu fréquemment, elles sont en aménorrhée ou allaitantes, ou encore stériles ou ayant du mal à tomber enceintes. Un quart souhaite avoir un enfant. Ces deux cas de figure sont éliminés de la Figure 7 , pour ne garder que les obstacles qui s'opposent à l'utilisation de méthodes modernes de contraception. 
Figure 6 : Raisons invoquées par les femmes qui ne pratiquent pas la contraception.

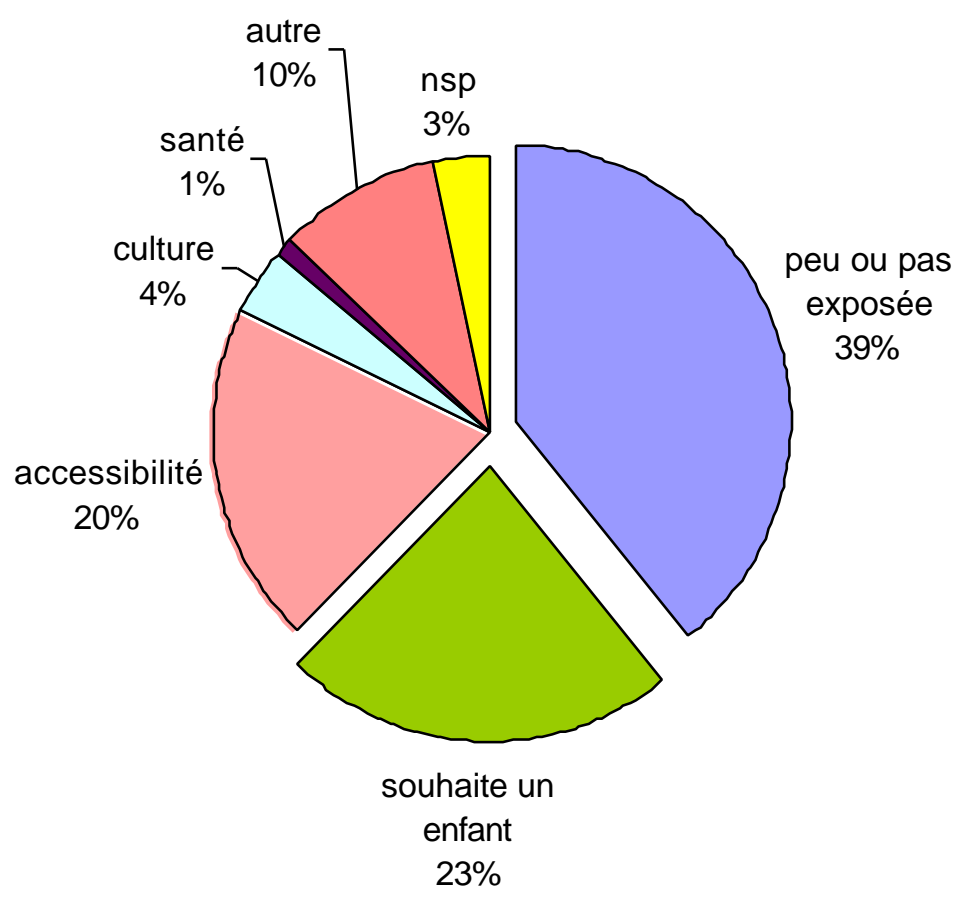

Source : LSC (UERD), enquête de base 1996.

La Figure 7 présente les obstacles invoqués par les femmes qui disent ne pas utiliser de contraception alors qu'elles sont conscientes d'être exposées au risque de tomber enceintes et qu'elles ne désirent pas d'enfant dans l'immédiat. 


\section{Figure 7 : Obstacles à l'utilisation de la contraception.}

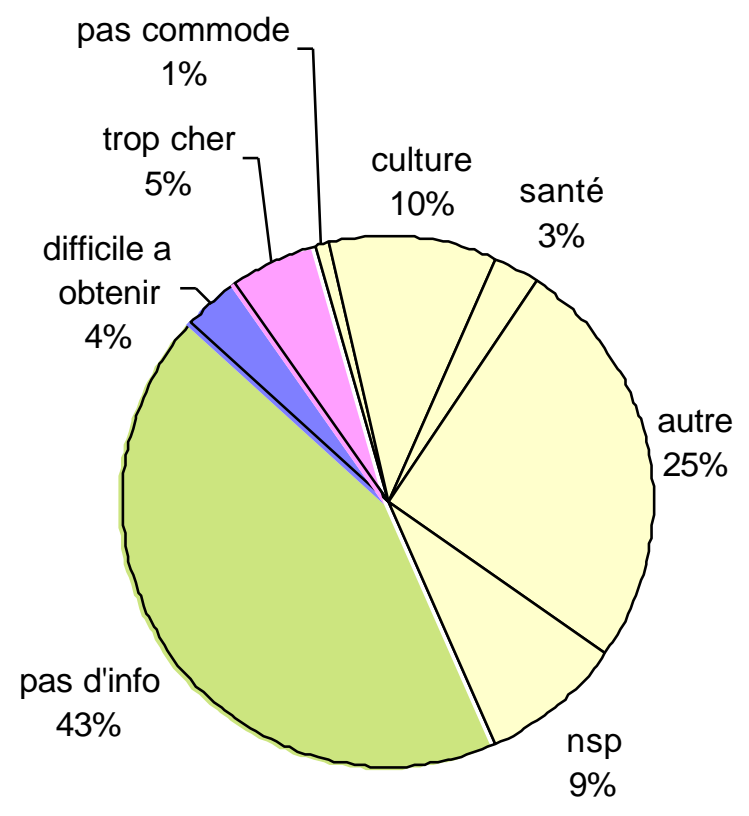

Source : LSC (UERD), enquête de base 1996. Les tranches hachurées correspondent au détail des obstacles liés à l'accessibilité présentés dans la Figure 6, inaccessibilité que l'intervention DBC doit tenter de résoudre.

Les obstacles liés à l'accessibilité des méthodes (absence d'information, disponibilité et coût) expliquent à eux seuls plus de la moitié des cas de non-utilisation de contraceptifs. C'est clairement sur ces aspects que doit intervenir le programme DBC, et plus particulièrement sur l'information. Les tranches blanches représentent les obstacles sur lesquels il est plus difficile d'agir, notamment les aspects plus «culturels » (religion, désapprobation de la femme ou du mari)

Précisons toutefois qu'une information accrue n'entraînera pas automatiquement une utilisation accrue de méthodes contraceptives. L'absence d'information n'est peut être pour nombre de femme qu'un prétexte facile à invoquer qui voile en fait des raisons plus profondes, voire inconscientes, d'ordre culturel, social ou personnel.

Cependant, on a constaté plus haut (0 page 41) que le niveau de connaissance de la majorité des femmes sur la contraception étaient limité, confirmant ainsi les besoins en matière de communication. 
2 Les façons dont les méthodes de contraception sont perçues limitent-elles leur utilisation?

Certaines femmes pensent que la pilule rend stérile.

$3 \quad$ Les méthodes contraceptives sont-elles perçues comme étant efficaces ?

Un des problèmes liés aux injections et à la pilule est que ces méthodes sont perçues comme étant trop efficaces, dans le sens que leurs effets contraceptifs peuvent perdurer au-delà de leur utilisation, voire rendre sterile.

Les injections, par leur mode d'utilisation, sont perçues comme les plus efficaces, tout comme les médicament injectables le sont sur les comprimés oraux (cf. chloroquine).

$4 \quad$ Les perceptions sur la contraception varient-elles avec l'âge, le sexe, la religion ?

L'objectif ici est de voir si les messages de promotion des contraceptifs doivent être formulés de manière différente en fonction de l'âge, du sexe ou de la religion. Il existe en effet des différences statistiquement significatives (test du $\chi^{2} ; \mathrm{P}<0,05$ ) entre religions concernant les connaissances et l'utilisation de contraceptifs modernes (Tableau 10), les opinions des femmes, et de ce qu'elles pensent être l'opinion de leurs maris (Figure 8). 
Tableau 10 : Connaissance et utilisation de la contraception des femmes mariées selon leur religion.

\section{Animistes Catholiques Protestante Musulmane}

Total

$\mathbf{S}$

$\mathbf{S}$

\section{Méthode moderne:}

\begin{tabular}{lrrrrr} 
n'en connaît aucune & 50,8 & 41,3 & 38,8 & 45,9 & 44,7 \\
en connaît au moins une & 49,2 & 58,7 & 61,2 & 54,1 & 55,3 \\
n'en a jamais utilisé & 96,2 & 92,7 & 89,6 & 93,6 & 93,5 \\
en a déjà utilisé & 3,8 & 7,3 & 10,4 & 6,4 & 6,5 \\
Méthode actuellement utilisée: & & & & & \\
aucune & 67,1 & 63,0 & 57,5 & 67,7 & 65,3 \\
moderne & 2,6 & 5,0 & 8,2 & 4,0 & 4,3 \\
traditionnelle & 21,8 & 21,5 & 24,6 & 17,2 & 20,0 \\
autre & 8,4 & 10,4 & 9,7 & 11,1 & 10,3 \\
otal (\%) & 100,0 & 100,0 & 100,0 & 100,0 & 100,0 \\
Total (n) & 417 & 836 & 134 & 935 & 2322 \\
\hline
\end{tabular}

Source : LSC (UERD), enquête de base 1996 et EDS/Burkina Faso, 1993. Femmes mariées uniquement.

Les Protestantes, et dans une moindre mesure les Catholiques connaissent et utilisent plus la contraception moderne. Les animistes en revanche sont plus «traditionaliste ». Cette situation est comparable à celle de Navrongo où les chrétiennes et les musulmanes sont deux fois plus susceptibles de recourir à la contraception que les animistes \{Phillips, Binka, et al. 1997 \#1060\}. Les Animistes sont en moyenne plus vieilles que les Chrétiennes. Or la probabilité de connaître ou d'avoir utilisé une méthode contraceptive croît avec l'âge. La différence entre religion concernant les CAP en matière de contraception est donc plus forte que ne le laisse supposer ces résultats.

Une plus forte proportion d'animistes désapprouve l'utilisation de la contraception (méthodes «naturelles » comprises). Toutefois, la grande majorité des femmes l'approuve (plus de 80\%). 


\section{Figure 8 : Opinions sur la contraception selon l'âge et la religion.}
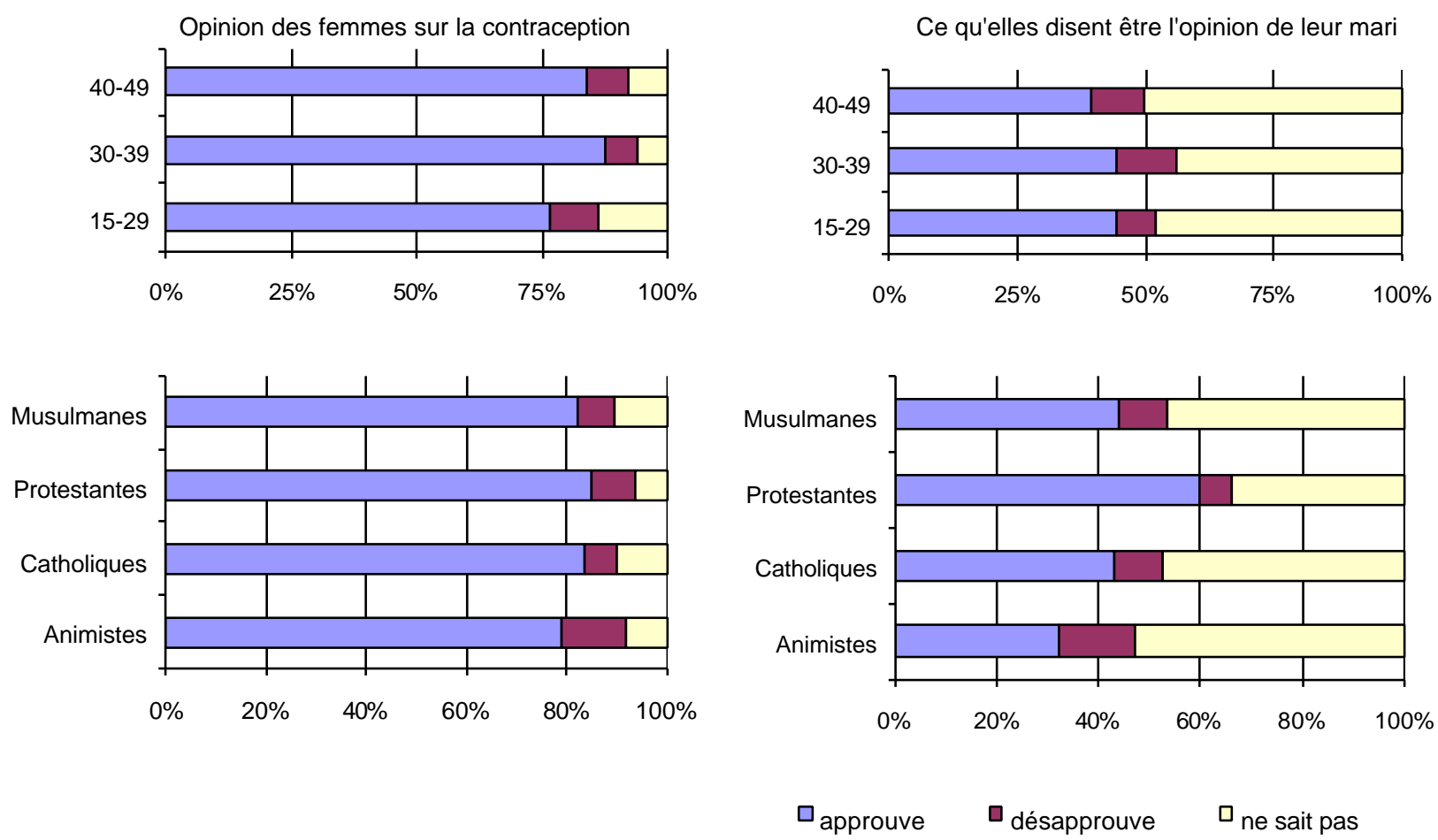

Source : LSC (UERD), enquête de base 1996 et EDS/Burkina Faso, 1993. Graphiques de gauche : toutes les femmes de 1549 ans ; graphiques de droite : femmes mariées uniquement. Méthodes de contraceptions naturelles comprises.

Au total, près de la moitié des femmes n'ont pas d'idées sur l'opinion de leur mari au sujet de la contraception. Ce résultat est cohérent avec le fait que les couples communiquent peu sur la fécondité. Ici encore, les différences entre religions sont fortes. L'effet âge, contrairement à ce qu'on a vu plus haut, peut expliquer une partie de cette différence. Les couples plus jeunes ont des rapports plus égalitaires (ou du moins dialoguent plus - cf. plus loin) que les couples plus âgés. Les femmes animistes, en moyenne plus âgées, ont donc moins de chance de connaître l'opinion de leur époux que les femmes chrétiennes.

En conclusion, les faibles différences d'opinion et de pratique entre religions ne justifie pas un ciblage différencier des activités de PF. En revanche, même si les différences d'opinions entre les adolescentes et les femmes plus âgées ne sont pas substantielles, les différences des besoins justifient des activités de PF différentes (cf. b.2 et la Figure 1 page 46).

Les entretiens de groupes permettent de préciser les obstacles culturels à l'utilisation de contraceptifs.

La terminologie Moore est ambigüe puisque les gens confondent couper les grossesses (kup rogm) et espacer les grossesses (yag rogm). Pour la plupart des participants aux entretiens de groupes, il n'y a pas 
de nuance entre «couper» et espacer. Ceci peut résulter de la croyance que les méthodes contraceptives empêchent définitivement d'accoucher (voir infra).

Comme tout au long du questionnement sur la procréation, la place centrale de Dieu et de l'ordre établi par lui se retrouve :

Ça dépend de ce que Dieu donne, c'est Dieu qui partage, c'est à lui

qu'appartiennent les enfants (jh, 2vh, fr).

Dans ce contexte, il n'est pas question d'intervenir dans cet ordre instauré par Dieu sous peine de conséquences interprétées comme des sanctions qui peuvent être néfastes, telles la stérilité ou la mort des enfants.

Le grand nombre d'inconvénients cités qui traduit des préoccupations diverses. Ces préoccupations sont d'une part liées au type de méthode choisie, d'autre part aux répercussions sociales de leur utilisation.

Beaucoup de groupes signalent qu'il y a des complications sans donner de plus amples détails (vh, jh, $2 \mathrm{hr}, 4 \mathrm{f}, \mathrm{jf})$. Les complications liées à la pilule sont :

- le risque d'oubli des comprimés qui entraîne la grossesse (vh, jh, hr, jf) ;

- les comprimés font grossir (jf) ; les comprimés peuvent provoquer la mort (jh).

Les préservatifs présentent aussi des inconvénients :

- $\quad$ risque d'avoir le sida avec les capotes (vh);

- absence de contrôle, ça se vend au hasard (2vh) ;

- $\quad$ difficile de suivre le mode d'emploi (hr),

- $\quad$ alors que selon les jeunes hommes : les capotes ne posent pas beaucoup de problèmes (jh).

Au niveau social, la présence de méthodes contraceptives entraine de nouvelles attitudes et comportements dans la mesure où les méthodes évitent de tomber en grossesse : cela favorise le vagabondage (2vh). Plusieurs groupes relèvent que l'utilisation des méthodes suppose l'entente entre les conjoints, sinon il y a la bagarre (vh, jf).

Les opinions défavorables à l'utilisation des méthodes contraceptives évoquent toute une série de considérations : sentiment de honte, relâchement des mœurs, dissension dans les foyers. Les arguments en faveur de l'ordre établi, celui de la nature guidée par Dieu se retrouvent: il faut se contenter de ce que Dieu donne (vh); avant c'était Dieu qui espaçait les naissances (vh) ; quand on est marié, il faut 
accoucher (jf) ; il faut se maîtriser jusqu'au mariage (jf). Le sentiment de honte se manifeste également à ce niveau : c'est honteux (vh, jh); cela entraîne des moqueries, des insultes $(5 \mathrm{f})$; ce n'est pas bien (2vh, jf). De plus, les méthodes contraceptives sont perçues comme un moyen de débauche pour les utilisateurs. La diminution du risque de grossesse entraînerait une licence sexuelle sans limite : ça entraîne l'infidélité (vh); il faut qu'il y ait une entente entre l'homme et la femme (h, 2hr); le mari se fâche si la femme utilise les comprimés (f) ; il faut le faire discrètement (3f) ; on dira que la femme veut chercher des hommes (5f). Enfin un groupe de jeunes gens affirment que certaines jeunes filles refusent qu'ils utilisent les préservatifs : certaines filles n'acceptent pas les garçons qui utilisent les capotes (jh).

$6 \quad$ L'intervention peut-elle modifier ces perceptions en trois ans?

Les perceptions changent lentement. Le dernier passage est prévu moins de quatre ans après le démarrage de l'intervention. Lorsque les perceptions sur la contraception auront été minutieusement identifiées et analysées, nous étudierons les facteurs et caractéristiques (âge, éducation, religion, etc.) des enquêtés favorables à la contraception et essaierons d'en déduire les changements attendus au bout de trois ans. Des entretiens approfondis valideront l'interprétation des données de l'enquête quantitative.

7 La modification des perceptions entraîne-t-elle une augmentation de l'utilisation des contraceptifs?

Il est encore trop tot pour se prononcer ; les perceptions n'ont pas encore suffisamment changé.

8 Les migrations et la proximité de Ouagadougou influencent-elles l'utilisation de la contraception $?$

Les quatre zones sont à proximité de Ouagadougou. Comme le montre le Tableau 11, plus d'une femme sur cinq est allée à Ouagadougou au moins une femme au cours des douze derniers mois, pour rendre visite à des parents ou amis, pour travailler, pour commercer, etc. et de ce fait sont exposés à un environnement plus propice à l'adoption de nouveaux comportements en matière de fécondité. On constate que c'est dans la zone DBC (zone B) que la proportion de femmes ayant séjourné à ouaga est la plus forte (près d'une femme sur trois, contre une femme sur six dans la zone C). 
Tableau 11: proportion des femmes de 15 à 49 ans qui ont séjourné à Ouaga lors des 12 derniers mois, selon la zone, l'âge et l'utilisation d'une méthode moderne de contraception.

\begin{tabular}{|c|c|c|c|c|}
\hline \multicolumn{5}{|c|}{ A séjourné à Ouaga lors des 12 derniers mois (\%) } \\
\hline & Oui & non & Total & $\mathrm{n}$ \\
\hline \multicolumn{5}{|l|}{ Zone } \\
\hline A & 18,5 & 81,5 & 100 & 600 \\
\hline B & 32,3 & 67,7 & 100 & 643 \\
\hline $\mathrm{C}$ & 13,4 & 86,6 & 100 & 768 \\
\hline $\mathrm{D}$ & 25,7 & 74,3 & 100 & 521 \\
\hline \multicolumn{5}{|l|}{ Age } \\
\hline $15-24$ & 24,0 & 76,0 & 100 & 940 \\
\hline 25-34 & 20,2 & 79,8 & 100 & 762 \\
\hline $35-49$ & 21,2 & 78,8 & 100 & 830 \\
\hline \multicolumn{5}{|c|}{ Utilise une méthode moderne } \\
\hline non & 20,3 & 79,7 & 100 & 2248 \\
\hline oui & 34,9 & 65,1 & 100 & 284 \\
\hline Total & 22,0 & 78,0 & 100 & 2532 \\
\hline
\end{tabular}

Source : LSC (UERD), enquête de passage 1998.

Or il y a un lien statistique entre le fait d'être allé à Ouaga et d'avoir utilisé une méthode contraceptive. En controlant l'effet âge ; la probabilité qu'une femme a d'utiliser ou d'avoir utilisé dans le passé une méthode contraceptive moderne est deux fois plus forte si elle est allé à Ouagadougou dans les douze derniers mois (Tableau 12).

Tableau 12: probabilité pour une femme de 15-49 ans d'avoir utilisé une méthode moderne de contraception selon les séjours à Ouaga.

Utilise une méthode moderne

Odds Ratio

2,1

Séjour à Ouaga*

Age

$25-34$ ans

$35-49$ ans

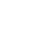

1,5

1,4
Std. Err

0,3

0,2

0,2

, 3

, 2

2

*au moins un séjour à ouaga dans les douze derniers mois précédant l'enquête ; n=2532

Source : LSC (UERD), enquête de passage 1998.

Il faut rappeler à cette occasion qu'il s'agit ici des déclaration des femmes. Or il est possible que les femmes qui se déplacent à Ouagadougou, parce qu'elles sont plus autonomes ou plus «modernes », ou encore plus âgées, avouent plus facilement utiliser une méthode contraceptives. 
Les migrations (vers Ouaga ou l'étranger) ont aussi d'autres effets sur les perceptions et comportements en matière de PF. Ainsi, la pyramide des âges montre une forte migration des hommes, ce qui pourrait en partie expliquer leur plus grande sensibilisation à la planification familiale. Enfin, les couples dont l'homme est fréquemment absent ou absent pendant de longues périodes, peuvent ne pas vouloir utiliser certains contraceptifs (pilule, stérilet).

\section{d. La prévalence de l'utilisation de la contraception est-elle faible parce que l'offre est inadéquate ?}

Le relation entre l'accessibilité de la contraception et son utilisation est ambiguë. Il est évident qu'une femme ne peut pas utiliser de contraceptifs s'ils lui sont inaccessibles. En revanche, une femme n'utilisera pas forcément un contraceptif s'il devient accessible. Or l'effet de l'offre de contraceptifs sur l'utilisation contraceptive constitue une hypothèse importante de l'intervention. L'accessibilité (en termes de coûts et de distance) de la contraception est maintenant analysée (l'acceptabilité culturelle figure dans le thème c). Les questions figurant ici se concentrent sur l'offre de contraception et les différentes méthodes de PF qui pourraient lui faire concurrence.

Il est important de rappeler que les données sur l'utilisation de la contraception se basent uniquement sur la déclaration des femmes et/ou de leurs époux. Or on sait que ces réponses sont sujettes à caution (cf. Navrongo)

$1 \quad$ Quelle était la prévalence contraceptive avant l'intervention?

Afin de comparer nos données avec celles de l'EDS, nous définissons la prévalence d'utilisation d'une méthode contraceptive donnée comme étant le pourcentage de femmes mariées qui utilisent cette méthode au moment de l'enquête. Les taux de prévalence contraceptive par zone avant l'intervention sont présentés dans le Tableau 13. 
Tableau 13 : Prévalence contraceptive par Zone, 1996.

Enquête de base (UERD-1996)

Zone

A $\quad$ B $\quad$ C
EDS-Burkina Faso (1993)

Lieu de résidence

D Total rural Ouaga. Burkina

\section{\% des femmes mariées}

\section{utilisant :}

\begin{tabular}{|c|c|c|c|c|c|c|c|}
\hline - une méthode moderne & 3,7 & 3,6 & 3,2 & 7,4 & 4,4 & 1,5 & 19,9 \\
\hline - pilule & 1,9 & 1,5 & 1,7 & 4,2 & 2,3 & $\mathbf{0 , 8}$ & 8,3 \\
\hline - DIU & 0,8 & 0,4 & 0,1 & 0,4 & 0,4 & $\mathbf{0 , 1}$ & 4,9 \\
\hline - injections & 0,2 & 0,2 & 0,1 & 0,2 & 0,2 & $\mathbf{0 , 0}$ & 0,9 \\
\hline - condoms & 0,7 & 1,5 & 0,9 & 2,7 & 1,4 & $\mathbf{0 , 3}$ & 4,5 \\
\hline - une autre méthode moderne & 0,2 & 0,0 & 0,3 & 0,0 & $\mathbf{0 , 1}$ & $\mathbf{0 , 3}$ & 1,3 \\
\hline - une méthode traditionnelle & 12,0 & 21,7 & 18,2 & 29,6 & 20,0 & 2,7 & 10,7 \\
\hline $\begin{array}{l}\text { - une méthode moderne ou } \\
\text { trad. }\end{array}$ & 15,8 & 25,3 & 21,4 & 37,0 & 24,4 & 4,2 & 30,6 \\
\hline
\end{tabular}

Source : LSC (UERD), enquête de base 1996 et EDS/Burkina Faso, 1993

On constate qu'en 1996, 4,4\% (102/2333; IC à 95\% : 3,5\%-5,2\%) de femmes mariées dans les quatre zones qui utilisaient une méthode moderne, essentiellement la pilule et le condom. C'est très inférieur à la prévalence constatée à Ouagadougou (près de 20\% en 1993) mais plus élevé que dans l'ensemble des zones rurales du pays (1,5\%). Il est possible que la proximité de Ouagadougou encourage l'utilisation de contraceptifs modernes (cf. c.8). La zone D se distingue des trois autres par un taux nettement plus élevé d'utilisation de pilule et de condom. On peut penser qu'il s'agit là d'un effet du programme Save the Children.

Toutefois, une prévalence de $4,4 \%$ est tout de même élevée pour une population à $100 \%$ rurale, où plus de neuf femmes sur dix sont analphabètes et ne sont jamais allées à l'école.

L'approche transversale peut aussi sous-estimer la pratique contraceptive. Bledsoe et al. remarquent, au sujet d'une étude en milieu rural en Gambie, que

Même si les femmes ne cherchent pas à limiter leur fécondité, il y en a plus qui pratiquent la contraception que ne le suggère les données transversales parce que la contraception se pratique dans le but d'espacer les naissances : pendant des courtes tranches de temps dans l'interval intergénésique ( [Bledsoe et al., 1994]). 
Par ailleurs, il est très possible que dans le Bazèga, comme à Navrongo, les femmes peuvent ne pas déclarer à l'enquêteur qu'elles utilisent une méthode contraceptive [Phillips et al., 1997]. Cela est particulièrement le cas des femmes qui prennent une méthode moderne à l'insu de leur mari. Il se pourrait donc que l'écart entre la zone des et les autres zones soit en partie liée au fait que les femmes de la zone $\mathrm{D}$ «avouent »plus facilement utiliser la pilule, rendue plus acceptable par les activités de PF de Save the Children.

Concernant les méthodes contraceptives traditionnelles, il est possible que beaucoup de femmes aient confondu abstinence périodique avec abstinence post partum, la seconde étant une pratique courante (cf. rapports des enquêtes de base) qui explique la longueur des intervalles intergénésiques observés.

$\rightarrow$ intégrer fréquences sur connaissances du cycle

\section{Figure 9 : Méthodes contraceptives utilisées par les femmes mariées pratiquant la PF.}

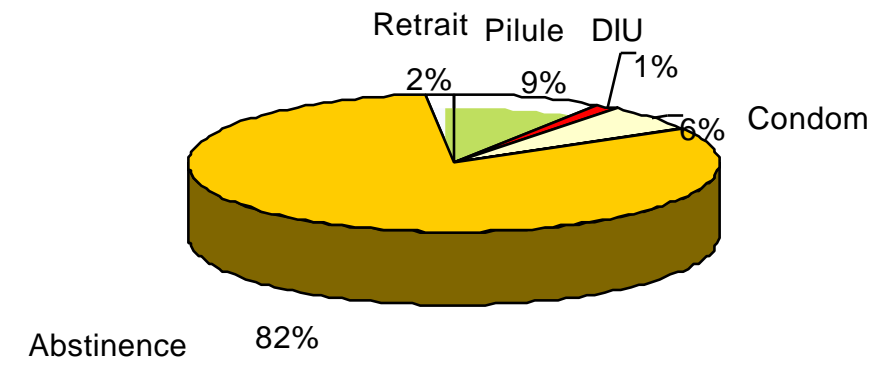

Source : LSC (UERD), enquête de base.

$2 \quad$ Le coût des contraceptifs a-t-il un effet sur leur niveau d'utilisation ?

Quelle est la somme que les femmes sont disposées à payer pour la pilule ? Il se pourrait que la pilule soit déjà à leur portée physique, mais jugée trop chère pour se la procurer : dans ce cas son utilisation n'augmentera pas même si la demande augmente. Le prix des différents contraceptifs est fixé par le programme, non par le marché (offre et demande). L'échelle des prix des pilules et des condoms vendus sur le marché dans le Bazèga constitue une bonne indication de ce que les femmes sont disposées à payer pour obtenir des contraceptifs. En effet, la stratégie DBC ne 
fonctionnera que si les contraceptifs vendus par le programme sont moins chers que ceux vendus sur le marché local.

3 L'utilisation des contraceptifs est-elle associée à la distance de la formation sanitaire ?

Si la distance physique s'avère un obstacle à l'utilisation de la contraception, alors il est cohérent de mettre en œuvre un schéma de distribution qui vise à rapprocher les contraceptifs des villageois. Toutefois, le système DBC mis en place dans la zone B est une stratégie de rapprochement parmi tant d'autres, telles que les pharmacies villageoises, les équipes mobiles de santé, etc. Si l'on constate une association négative entre la distance de la formation sanitaire et l'utilisation de la contraception, cela ne veut pas dire pour autant que la stratégie DBC soit pertinente, cela indique seulement que ce genre de distribution de services qui consistent à rapprocher les prestations de la communauté pourrait augmenter l'utilisation des contraceptifs.

Y a-t-il une offre pour d'autres méthodes de maîtrise de la fécondité ?

S'il existe d'autres méthodes que les contraceptifs pour réguler la fécondité (avortement, méthodes traditionnelles...), et que ces méthodes sont plus accessibles (en termes d'acceptabilité culturelle, de distance, de coût), alors l'utilisation des contraceptifs n'augmentera pas même si l'offre de contraceptifs est améliorée.

\subsubsection{La situation au Bazèga justifie-t-elle une intervention en planification familiale?}

Les données de l'enquête de base sont comparées aux résultats issus de l'enquête EDS 1993 pour le Burkina rural. Le choix de l'emplacement de l'intervention se justifie en terme de santé publique si la situation dans ces zones est au moins égale ou pire que celle des autres provinces rurales. Nous ne considérons pas les autres éléments qui peuvent avoir été pris en compte pour le choix des zones (logistique, priorités institutionnelles, disponibilités des moyens, accessibilité géographique, organisation communautaire préexistante, etc.). Les zones de la DBC sont comparées à "une province moyenne rurale" et non à des provinces individuelles : si la situation dans les zones de la stratégie DBC s'avérait plus mauvaise que la moyenne rurale n’implique que ces zones soient parmi les plus défavorisées du pays.

Cette question est largement éthique : les ressources en santé publique doivent être mobilisées prioritairement au profit des populations les plus défavorisées. On est aussi en mesure de penser que si la cause principale d'une fécondité élevée est l'inaccessibilité de contraceptifs, alors la situation au Bazèga ne sera pas parmi la plus défavorable du fait de la proximité de Ouagadougou. L'effet de l'intervention sur l'utilisation de contraceptifs et sur la fécondité au Bazega serait de ce fait moins fort que dans une province plus isolée. 
Par ailleurs, la population soumise à un projet pilote ne dois pas être susceptible de mieux accepter l'intervention qu'une autre. Si c'était le cas, les résultats de l'expérimentation ne seraient pas généralisables. Ceci est particulièrement vrai dans le cas des interventions à base communautaire où l'organisation de celle-ci est une condition sine qua non de succès.

\section{a. La fécondité est-elle plus élevée au Bazèga que dans l'ensemble du Burkina Faso rural ?}

1 Le taux de fécondité global dans le Bazèga est-il significativement plus élevé que dans l'ensemble du Burkina Faso rural ?

Comme le montre la Figure 10, les taux de fécondité par âge au Bazèga sont légèrement inférieurs à ceux des zones rurales (en 1993), à l'exception des deux classes d'âges extrêmes où les taux sont plus proches de ceux de la capitale. On remarque aussi que les taux de fécondité des 15-19 ans (100 pour mille) est aussi faible dans le Bazega qu'à Ouagadougou. Ceci s'explique le taux encore élevé de femmes célibataire, célibat probablement provoqué en grande partie à la forte migration des hommes.

Figure 10 : Taux de fécondité par âge dans le Bazèga, à Ouaga et en zone rurale.

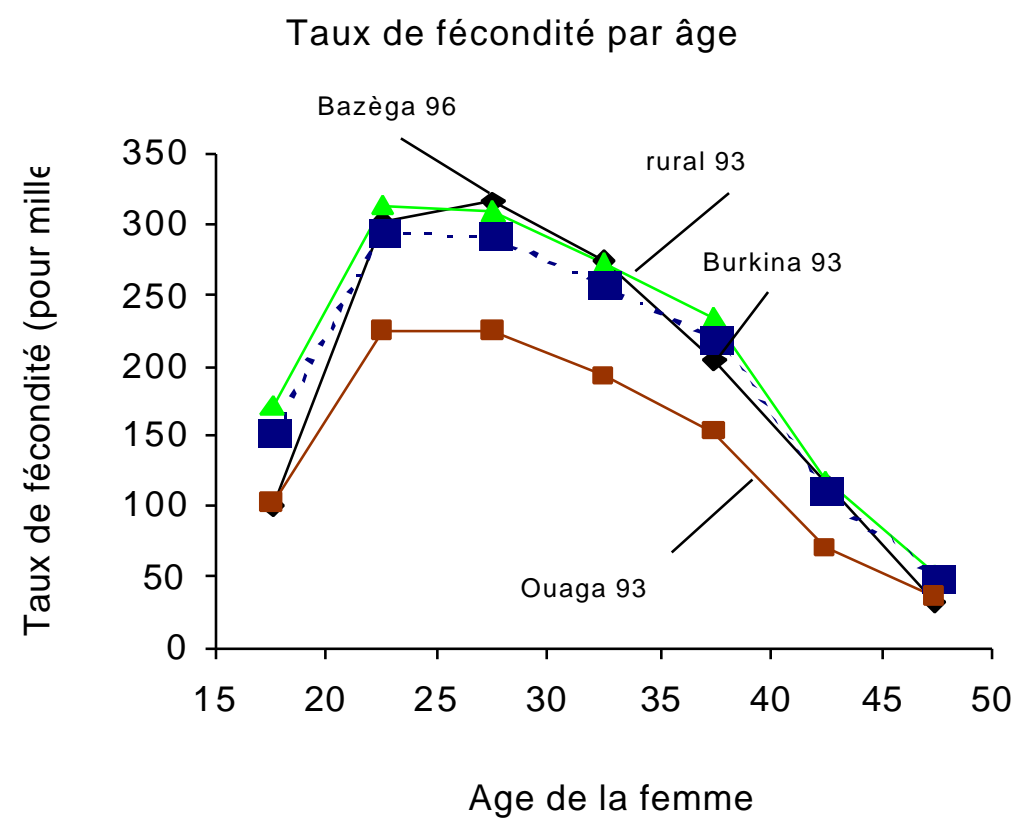

Source : LSC (UERD), enquête de base 1996 et EDS/Burkina Faso, 1993

En conséquence, l'indice synthétique de fécondité (ISF) au Bazèga est de 6,7 enfants par femmes, contre 7,3 en zone rurale et 4,7 à Ouagadougou (Tableau 14). L'ISF est inférieur à la descendance finale au Bazèga, puisque celle-ci est de 7,4 enfants par femme (Tableau 6). Ceci pourrait s'expliquer par une baisse de la 
fécondité. Il est plus probable, cependant, de l'attribuer à un effet de sélection de l'enquête par lequel les femmes avec beaucoup d'enfant sont plus souvent à la maison que celles qui en ont eu moins et qui sont donc plus mobiles, et qui se déplacent à Ouagadougou.

Tableau 14 : Indicateurs de fécondité par zones.

\begin{tabular}{|c|c|c|c|c|c|c|c|c|}
\hline & \multicolumn{4}{|c|}{ Enquête de base (UERD-1996) } & \multicolumn{4}{|c|}{ EDS-Burkina Faso (1993) } \\
\hline & \multicolumn{4}{|c|}{ Zone } & \multirow[b]{2}{*}{ Total } & \multicolumn{3}{|c|}{ Lieu de résidence } \\
\hline & A & B & $\mathrm{C}$ & $\mathrm{D}$ & & rural & Ouaga. & Burkina \\
\hline \multicolumn{9}{|l|}{ Indicateurs de fécondité } \\
\hline Taux de natalité ${ }^{1}$ & 40 & 35 & 41 & 69 & 38 & 43 & 37 & 43 \\
\hline $\mathrm{TGFG}^{2}$ & 217 & 189 & 229 & 186 & 206 & 249 & 156 & 233 \\
\hline Indice synthétique de fécondité ${ }^{3}$ & 6,8 & 6,3 & 7,6 & 6,1 & 6,7 & 7,3 & 4,7 & 6,9 \\
\hline
\end{tabular}

On constate donc que les trois indicateurs de fécondité présentés plus haut montrent que la situation au Bazèga relative à la fécondité est plus favorable que dans la moyenne des zones rurales du pays. Cette province est en avance sur les autres provinces rurales, sans doute grâce à sa proximité à la capitale. (Le taux de natalité élevé observé dans la zone D est probablement du a la petite taille de l'échantillon au dénominateur)

2 Les connaissances, aptitudes et pratiques en matière de PF sont-elles moins favorables à une baisse de la fécondité dans les zones LSC que dans l'ensemble du Burkina Faso rural?

Cette question a déjà été en partie répondue en 1.1.1b.1. La même proportion de femmes mariées a entendu parler d'au moins une méthode moderne au Bazèga que dans l'ensemble des zones rurales (55\% contre 57\%, cf. Tableau 7, page 42). Les résultats de l'EDS par méthode et par zone de résidence n'étant pas disponibles, nous comparerons la situation du Bazèga avec celle de l'ensemble du pays, fortement influencé à la hausse par la population urbaine. Or ici on constate que le Bazèga est comparable avec l'ensemble du pays, ce qui indique probablement, ici aussi, une situation plutôt plus favorable parmi les zones rurales. 
3 La demande non satisfaite de PF est-elle plus importante dans les zones LSC que dans l'ensemble du Burkina Faso rural ?

Reprenons les résultats présentés dans le Tableau 9 ; page 45. La situation dans le Bazèga est intéressante car il y existerait plus de besoins en planification familiale que dans l'ensemble des zones rurales : 42,1\% des femmes auraient besoin, contre 28,5\% ailleurs. Ceci pourrait s'expliquer par le fait qu'une plus grande proportion de femmes au Bazèga reconnait l'intérêt d'espacer et, dans une moindre mesure, de limiter les naissances. En revanche, la prévalence d'utilisation y est légèrement supérieure, suggérant qu'une meilleure accessibilité des méthodes puisse en effet en accroître l'utilisation.

4 La prévalence de l'utilisation de la contraception est-elle plus faible dans les zones LSC que dans l'ensemble du Burkina Faso rural ?

Non. Cf. 1.1.1d.1 et Tableau 13, page 58.

\section{b. La situation démographique et sanitaire est-elle plus difficile au Bazèga que dans l'ensemble du Burkina Faso rural ?}

Le Tableau 15 permet de comparer les valeurs des principaux indicateurs de santé dans les zones LSC avec celles du reste du pays. Les différences entre zones des taux de mortalité sont en grande partie due à la taille réduite des échantillons et ne seront donc pas analysées. En revanche, les indicateurs entre la population LSC et celles du Burkina rural montrent que la première n'est pas particulièrement défavorisée par rapport à la seconde. 
Tableau 15 : Principaux indicateurs de santé, zones LSC.

\begin{tabular}{|c|c|c|c|c|c|c|c|c|}
\hline & \multicolumn{4}{|c|}{ Enquête de base (UERD-1996) } & \multicolumn{4}{|c|}{ EDS-Burkina Faso (1993) } \\
\hline & \multicolumn{4}{|c|}{ Zone } & \multirow[b]{2}{*}{ Total } & \multicolumn{3}{|c|}{ Lieu de résidence } \\
\hline & A & $\mathrm{B}$ & $\mathrm{C}$ & $\mathrm{D}$ & & rural & Ouaga. & Burkina \\
\hline Mortalité infantile & 114 & 109 & 140 & 94 & 117 & 113 & 83 & 108 \\
\hline Mortalité infanto-juvénile (0-4ans) & 178 & 151 & 209 & 225 & 192 & 214 & 150 & 204 \\
\hline \multicolumn{9}{|l|}{ Prevalence de l'excision } \\
\hline parmi toutes les femmes & 95,2 & 83,3 & 84,8 & 96,4 & 89,6 & & & \\
\hline parmi les $15-24$ ans & 94,1 & 79,3 & 78,5 & 96,3 & 86,8 & & & \\
\hline parmi les $35-49$ yrs & 96,0 & 85,6 & 86,5 & 99,0 & 91,4 & & & \\
\hline \multicolumn{9}{|l|}{ Nombre de consulations prénatales } \\
\hline aucune & 6,3 & 6,3 & 18,6 & 7,2 & 10,3 & & & 40,0 \\
\hline 1 & 8,6 & 8,5 & 5,7 & 8,8 & 7,7 & & & 5,0 \\
\hline $2-3$ & 57,0 & 47,2 & 36,1 & 54,6 & 48,0 & & & 29,4 \\
\hline 4 ou plus & 27,6 & 36,9 & 36,9 & 29,4 & 32,8 & & & 22,6 \\
\hline NSP / Non disponible & 0,5 & 1,1 & 2,7 & 0,0 & 1,2 & & & 3,1 \\
\hline \multicolumn{9}{|l|}{ Nombre d'injections antitétanique } \\
\hline 0 & 28,2 & 36,0 & 46,7 & 45,3 & 39,0 & 40,7 & 7,1 & 36,1 \\
\hline 1 & 27,8 & 28,7 & 22,0 & 19,6 & 24,4 & 20,4 & 20,0 & 20,3 \\
\hline 2 ou plus & 43,1 & 33,5 & 31,3 & 35,2 & 35,9 & 37,3 & 70,2 & 41,8 \\
\hline NSP / Non disponible & 1,0 & 1,8 & 0,0 & 0,0 & 0,7 & 1,6 & 2,6 & 1,7 \\
\hline \multicolumn{9}{|l|}{ Bébé nourri au"premier lait" } \\
\hline $\begin{array}{l}\text { (dernier enfants nés moins de } 18 \text { mois } \\
\text { avant l'enquête) }\end{array}$ & 42,7 & 65,3 & 39,8 & 88,7 & 57,0 & 45,9 & 67,7 & 48,2 \\
\hline Prevalence de diarrhée & 16,9 & 24,9 & 20,8 & 25,0 & 21,8 & 20,6 & 19,4 & 20,3 \\
\hline Prevalence de paludisme & 16,5 & 22,9 & 14,0 & 13,8 & 16,6 & & & \\
\hline
\end{tabular}

La mortalité infantile et juvénile est a la fois un indicateur de santé et un déterminant de la fécondité. La mortalité infantile et juvénile a un effet sur la fécondité. Un niveau de mortalité élevé pousse les parents à concevoir plus d'enfants afin de compenser les effets de la mortalité sur leur descendance. Par ailleurs, de façon plus mécanique, une baisse de la mortalité infantile élimine certains des intervalles intergénésiques courts, diminuant ainsi la fécondité. 
1 La mortalité infantile est-elle plus élevée dans les zones LSC que dans l'ensemble du Burkina Faso rural ?

Non. (cf. Tableau 15)

2 La mortalité infanto-juvénile est-elle plus élevée dans les zones LSC que dans l'ensemble du Burkina Faso rural ?

Non. (cf. Tableau 15) 


\subsection{La mise en cuvre de l'intervention est-elle satisfaisante?}

Dans cette section, nous comparons les objectifs opérationnels aux résultats effectivement atteints (en terme d'outputs). Quand les activités n'ont pas objectifs quantitatifs fixés, nous avons avons estimé le niveau d'activité auxquels ont peut raisonnablement s'attendre après 18 mois d'intervention ${ }^{10}$. L'analyse des résultats opérationnels indiquera les voies et mécanismes par lesquels l'intervention a eu un effet (0), ainsi que la faisabilité de chacune des deux stratégies (0).

\subsubsection{Couverture de l'intervention}

La mise en œuvre satisfaisante de l'intervention est une condition sine qua non du succès de la stratégie DBC. Nous entendons par «mise en œuvre satisfaisante de l'intervention » l'exécution effective de tous les mécanismes qui permettent d'exposer la population de la zone B au travail de sensibilisation des agents DBC. Cette exposition se mesure par l'étendue de la couverture (proportion de concessions et de ménages touchés par le travail des agents DBC) et l'intensité d'exposition (ce que les individus ont retenu des message des agents DBC).

1 Quelle est la proportion de ménages de la zone B qui a été visitée par les agents DBC ?

Hypothèse d'évaluation : Dans la zone B, il y a 84 agents DBC pour un nombre total de 5527 ménages (soit 65 ménages par agent DBC) et 9318 femmes en âge de procréer (soit 110 femmes par agent DBC). Nous nous attendons à ce que $75 \%$ (est.) des ménages et $60 \%$ (est.) des femmes éligibles aient été visitées au moins une fois, soit 50 ménages et 66 femmes.

Résultats de l'évaluation :

Les résultats opérationnels sont loin d'atteindre les objectifs quelque soit l'unité d'analyse (individu, ménage, concession) ainsi que l'indique le Tableau 16 qui présente les taux de couverture par formation sanitaire.

\footnotetext{
${ }^{10}$ Ces estimations UERD, suivie de (est), sont basées sur l'énumération et de l'enquête de 1996. Elles ont été validées par
} la DSF lors de l'atelier des 17-19 fevrier 1998. 
Tableau 16 : Statistiques de couvertures et de rendement DBC par FS .

\begin{tabular}{rrrr}
\hline Unités visitées & Total unités & Couverture & Rendement \\
$(\mathrm{n})$ & $(\mathrm{N})$ & $(\mathrm{n} / \mathrm{N})$ & $(\mathrm{n} / \mathrm{ADBC}) / \mathrm{t}$ \\
\hline
\end{tabular}

\section{Femmes}

$\begin{array}{lrrrr}\text { Bassemyam } & 28 & 147 & 19,0 & 17,5 \\ \text { Komki-Ipala } & 22 & 140 & 15,7 & 34,4 \\ \text { Komsilga } & 53 & 228 & 23,2 & 28,8 \\ \text { Lougsi } & 12 & 78 & 15,4 & 25,0 \\ \text { Tintilou Nord } & 12 & 81 & 14,8 & 16,7 \\ \text { Vipalogo } & 14 & 153 & 9,2 & 9,7 \\ \text { Total } & 141 & 827 & 17,0 & 21,0\end{array}$

\section{Ménages}

$\begin{array}{lrrrr}\text { Bassemyam } & 27 & 95 & 28,4 & 16,9 \\ \text { Komki-Ipala } & 18 & 66 & 27,3 & 28,1 \\ \text { Komsilga } & 43 & 123 & 35,0 & 23,4 \\ \text { Lougsi } & 9 & 45 & 20,0 & 18,8 \\ \text { Tintilou Nord } & 11 & 44 & 25,0 & 15,3 \\ \text { Vipalogo } & 14 & 82 & 17,1 & 9,7 \\ \text { Total } & 122 & 455 & 26,8 & 18,2\end{array}$

\section{Concessions}

\begin{tabular}{lrrrr} 
Bassemyam & 24 & 81 & 29,6 & 15,0 \\
Komki-Ipala & 16 & 47 & 34,0 & 25,0 \\
Komsilga & 43 & 111 & 38,7 & 23,4 \\
Lougsi & 9 & 34 & 26,5 & 18,8 \\
Tintilou Nord & 10 & 38 & 26,3 & 13,9 \\
Vipalogo & 13 & 73 & 17,8 & 9,0 \\
Total & 115 & 384 & 29,9 & 17,1 \\
\hline
\end{tabular}

Rendement par DBC: nombre moyen d'unité visitée par un ADBC les 12 derniers mois precedant l'enquête.

Nombre de DBC par FS : Bassemyam:20; Komki-Ipala:8; Komsilga:23; Lougsi:6; Tintilou Nord:9; Vipalogo:18.

t: taux de sondage $=1 / 12$

Source : LSC (UERD), enquête de passage 1998.

Le taux de rendement $R$ des agents DBC est calculé en divisant le nombre de concessions visitée par le nombre d'agents DBC. Connaissant le taux de sondage de l'enquête, on peut estimer le rendement des agents DBC de chaque formation sanitaire avec la formule :

$$
R_{f s}=\frac{1}{t} \cdot \frac{c}{N_{f s}}
$$


Où $N_{f s}$ est le nombre d'ADBC travaillant dans l'aire de la formation sanitaire $f s$ et C le nombre de concession dans l'échantillon. Le taux de sondage $t$ dans la zone B est de $1 / 12^{\mathrm{e}}$. Les rendements des ADBC sont présentés dans la colonne de droite du Tableau 16. Ces résultats sont à comparer avec les statistiques de suivi des DBC établies par la DSF.

Le rendement moyen par ADBC toute formations sanitaires confondue est de 21 femmes visitées (17\%) en 12 mois, ce qui représente moins du tiers des objectifs fixés au départ. Par FS ce rendement n'est guère meilleur. la FS de Vipalogo enregistre le rendement le plus faible avec seulement 9,2\% de femmes visitées au cours des 12 derniers mois par les 18 ADBC de l'aire.

Il est clair que ces résultats médiocres se retrouvent en terme de ménages et de concessions visitées (cf. Tableau 16). Ainsi, les ADBC ont en moyenne visité 18,2 ménages chacun ; ce qui représente moins de deux ménages par mois et par ADBC !

A qui les $\mathrm{ADBC}$ rendent elles visite? Les beneficiaires des visites des ADBC sont avant tou les femmes de 25 à 34 ans. Ce sont en effet elles qui sont le plus à même de vouloir une méthode pour espacer les naissances. Les plus jeunes sont moins accessibles, plus timides, et moins motivées par la PF (cf premiere partie). Les plus vieilles, en revanche, sont plus intimidantes et certaines n'ont plus de rapports sexuels. Il n'est donc pas surprenant que les femmes de 25-34 soient mieux couvertes par la DBC (Tableau 17). Or les jeunes filles sont les plus a risques d'avoir recours à un avortement suite à une grossesse pre-nuptiale. En terme de santé de la reproduction, la négligence de ce groupe d'âge est une sérieuse lacune.

Tableau 17 : Proportion des femmes ayant reçu au moins une visite selon le groupe d'âge.

\begin{tabular}{|c|c|c|c|}
\hline & femmes visitées (n) & $\begin{array}{l}\text { Prop de femmes } \\
\text { visitées (\%) }\end{array}$ & IC à 95\% \\
\hline \multicolumn{4}{|l|}{ Groupe d'âge } \\
\hline $15-24$ ans & & 13,1 & $9,4-16,8$ \\
\hline $25-34$ ans & & 26,1 & $19,7-32,5$ \\
\hline $35-49$ ans & & 16,0 & $11,8-19,8$ \\
\hline $15-49$ ans & 141 & 17,0 & $14,5-19,6$ \\
\hline
\end{tabular}

Source : LSC (UERD), enquête de passage $1998 ; n=827$ femmes 
2 Quelle est la proportion d'individus qui ont visité leur FS au cours des douze derniers mois ? Sont-ils allés à une autre formation sanitaire ?

Les individus peuvent se rendre à la formation sanitaire pour d'autres motifs que la planification familiale (habituellement pour des soins ou des vaccinations). Cela leur donne l'occasion de voir les améliorations dans les zones A et B et d'être ainsi exposés aux activités de planification familiale.

Le Tableau 18 présente les statistiques de la fréquentation des FS par les femmes, à travers lesquelles on peut apprécier l'intensité de l'exposition des femmes de 15 à 49 ans aux formations sanitaires.

Tableau 18 : Fréquentation des FS dans les quatre zones.

\begin{tabular}{lrrrrr}
\hline & \multicolumn{7}{c}{ Zones } \\
& $\mathrm{A}$ & $\mathrm{B}$ & $\mathrm{C}$ & $\mathrm{D}$ & Total \\
\hline \% de femmes exposées & & & & & \\
$\quad$ à la FS de référence & 48,6 & 54,2 & 43,9 & 56,2 & 50,3 \\
$\quad$ exclusivemt à une autre FS & 9,5 & 15,7 & 11,6 & 7,0 & 11,2 \\
$\quad$ total & 58,1 & 69,9 & 55,5 & 63,2 & 61,5 \\
\% de femmes non exposées & 41,8 & 29,8 & 44,3 & 36,6 & 38,3 \\
nombre total de femmes (n) & 570 & 605 & 672 & 484 & 2331 \\
\hline
\end{tabular}

Source : LSC (UERD), enquête de passage 1998.

Nous avons distingué trois types d'exposition :

- l'exposition aux formations sanitaire de reference. Il s'agit de la proportion de femmes qui déclarent avoir fréquenté leur FS au cours des 12 derniers mois précedant l'enquête. On constate que les taux de fréquentation sont de $50 \%$ en moyenne dans les quatre zones.

- l'exposition exclusive à une autre FS que la FS de reference. Il s'agit de la proportion de femmes qui ne sont pas allées à leur formation sanitaire de reference mais à une autre formation sanitaire. Ainsi, au total $11 \%$ des femmes (261 femmes sur 2331) ont préféré aller dans une formation sanitaire autre que la $\ll$ leur ».

- Le taux d'exposition totale aux FS est la proportion de femmes qui est allée a une FS les douze derniers mois, que ce soit la FS de référence ou non.. En moyenne, 61,5\% des femmes ont exposées aux formations sanitaires. Dans les zones d'intervention, ce taux va de 58,1\% dans la zone A à 69,9\% dans la zone B (taux le plus élevé des 4 zones). 
1ere remarque. Les femmes de 15 à 49 ans sont les plus susceptibles d'aller à une FS. Ce sont elles qui ont le plus besoin des services de santé. Elle vont aux visites prénatales, elle s'occupent des enfants, (vaccinations, pesée, et soins en cas de maladies infanto-juvéniles et maternelles). Un taux de couverture de $50 \%$ des femmes en âge de procréation n'est donc pas étonnant. Il n'est pas un indicateur de satisfaction, ni une indication de frequentation élevée des services. En revanche, on peut supposer que sur deux ou trois ans, la quasi totalité des femmes frequenteront une FS et pourront être exposée aux messages qui y sont diffusés (affiches, causerie, conseils).

$2^{\mathrm{e}}$ remarque. A la question de savoir pourquoi des femmes sont allée a une autre FS que la leur, la tres grande majorité $(78,5 \%)$ d'entre elles avancent la raison de la proximité (69,5\% dans la zone B). C'est dire que certains villages demeurent éloignés des formations sanitaires. Cela justifie la tentative de rapprocher les services de la population (la stratégie DBC est une des approches possible). Les motifs qui ont conduit ces femmes à visiter une autre formation sanitaire sont par ordre d'importance : la consultation prénatale $(31 \%)$, la consultation des nourrissons $(19,9 \%)$ et la vaccination $(14,9 \%)$. La PF n'est un motif de déplacement dans une FS que pour $1 \%$ des femmes.

3 Pour quels motifs ces femmes ont-elles visité leur FS ?

Cette question a pour but d'identifier les principales raisons de fréquentation des formations sanitaires et la part de consultation pour la planification familiale. Les résultats de l'enquête de 1998 sont présentés dans le Tableau 19. 
Tableau 19 : Motifs de fréquentation des FS de reference au cours des 12 derniers mois, par zone.

\begin{tabular}{lccrrr}
\hline & \multicolumn{5}{c}{ Zone } \\
& A & B & C & D & Total \\
\hline Motifs de la visite (\%) & & & & \\
Consultations & & & & \\
prenatale & 32,1 & 36,0 & 33,2 & 29,0 & 32,8 \\
nourissons & 31,0 & 45,1 & 27,1 & 33,1 & 34,5 \\
PF & 11,2 & 9,1 & 3,7 & 9,9 & 8,4 \\
vaccination & 41,9 & 44,8 & 38,3 & 45,6 & 42,7 \\
mere enfant & 15,9 & 14,9 & 16,9 & 14,7 & 15,6 \\
autre & 3,2 & 2,4 & 2,4 & 3,3 & 2,8 \\
& 277 & 328 & 295 & 272 & 1172 \\
\hline
\end{tabular}

NB: Le total des pourcentages est superieur a 100 car on peut aller à une FS pour plusieurs raisons Source : LSC (UERD), enquête de passage 1998.

Les raisons évoquées par les femmes sont multiples. Les principales sont la vaccination, les consultations de nourrissons et les consultations prénatales. Moins d'une femme sur 10 évoque la PF comme étant l'objet d'une visite à la FS. Dans la zone B, ce pourcentage $(9,1 \%)$ est assez logique au vu du taux de prévalence contraceptive de 8\% enregistré par ailleurs en 1998.

\subsubsection{Intensité de l'exposition}

$1 \quad$ Est-ce que l'enquêtée connaît son agent DBC (Zone B)?

Hypothèse d'évaluation : l'enquêtée connaît son agent DBC si elle se souvient de son nom (ces noms sont comparés à ceux qui figurent dans la liste des agents qui relèvent de chaque aire de formation sanitaire)

L'intensité de l'exposition se mesure par les connaissances que les individus exposés à la DBC ont de leurs activités. Ainsi, on demande a chaque personne enquêtée si elle a entendu parlé des ADBC et si elle connaît l'ADBC de son village. On suppose que si elle se souvient du nom de l'ADBC elle la connait. Les noms donnés sont comparés à ceux de la liste des ADBC de chaque formation sanitaire. 
Les résultats obtenus sont encourageants. En effet, sur les 827 femmes de l'échantillon de la zone B, 448 (54 \%) déclarent avoir entendu parler des ADBC. Sur ces 448 femmes, 407 (91\%) déclarent connaitre leur $\mathrm{ADBC}^{11}$. Ces résultats témoignent-ils de l'intérêt que la population accorde à l'intervention ?

Ces résultats seuls ne permettent pas de conclure sur l'intensité de l'exposition à l'intervention DBC. La population peut connaître l'ADBC du village sans pour autant comprendre sa fonction. C'est pourquoi nous avons demandé aux enquêtées qui déclarent avoir entendu parler des ADBC quelle était, pour elles, la finalité du travail des ADBC. Ainsi, sur les 448 femmes sus mentionnées, 237 (53\%) pensent comprendre la finalité de la DBC. Lorsqu'on leur demande de la preciser, 184 (77,6 \%) répondent qu'elle consiste à « promouvoir la santé dans le village ».

2 Combien de fois l'agent DBC a-t-il visité l'enquêtée ces douze derniers mois ?

Vu le nombre de thèmes qu'ont à aborder les $\mathrm{ADBC}$, la fréquence des visites est un élément important dans la transmission des messages. En effet, le rendement des ADBC en terme de ménages, concessions ou femmes couvertes n'est significatif que si l'ADBC arrive à faire passer ses messages au cours de ses visites.

Le tableau ci-dessus présente le nombre de visites d'un ADBC chez les femmes qui ont reçu au moins une visite les douze derniers mois, par formation sanitaire.

Tableau 20 : Frequences et distribution des visites ADBC par formation sanitaire.

\begin{tabular}{|c|c|c|c|c|c|c|c|c|c|c|}
\hline & \multirow[b]{2}{*}{$\begin{array}{l}\text { Nombre } \\
\text { d'ADBC }\end{array}$} & \multirow[b]{2}{*}{$\begin{array}{l}\text { Femmes } \\
\text { visitées }\end{array}$} & \multirow[b]{2}{*}{$\begin{array}{l}\text { Nombre moyen de } \\
\text { visites reçues }\end{array}$} & \multicolumn{5}{|c|}{ Centiles } & \multicolumn{2}{|c|}{ Rendement par DBC* } \\
\hline & & & & 5 & 10 & 50 & 90 & 95 & $\begin{array}{l}\text { Femmes } \\
\text { visitées }\end{array}$ & $\begin{array}{c}\text { Nombre } \\
\text { moyen de } \\
\text { visites }\end{array}$ \\
\hline $\begin{array}{l}\text { Formations } \\
\text { sanitaires }\end{array}$ & & & & & & & & & & \\
\hline Bassmyam & 20 & 28 & 3,0 & 1,0 & 1,0 & 2,0 & 6,0 & 10,0 & 17,5 & 51,9 \\
\hline Komki-Ipala & 8 & 22 & 1,3 & 1,0 & 1,0 & 1,0 & 2,0 & 2,0 & 34,4 & 45,3 \\
\hline Komsilga & 23 & 53 & 2,8 & 1,0 & 1,0 & 3,0 & 5,0 & 7,0 & 28,8 & 82,1 \\
\hline Lougsi & 6 & 12 & 2,5 & 1,0 & 1,0 & 2,5 & 3,0 & 6,0 & 25,0 & 62,5 \\
\hline Tintilou & 9 & 12 & 1,8 & 1,0 & 1,0 & 1,0 & 3,0 & 5,0 & 16,7 & 29,2 \\
\hline
\end{tabular}


Rendement par DBC: nombre de visites par un ADBC les 12 derniers mois precedant l'enquête.

$*$ dans la population, soit $\mathrm{n} / \mathrm{t}$; $\mathrm{t}$ étant le taux de sondage $=1 / 12$

Source : LSC (UERD), enquête de passage 1998.

Au total, les femmes visitées par les ADBC, l'ont été en moyenne 2,7 fois en 12 mois (écart type : 3,1). Cette moyenne générale cache des disparités entre formations sanitaires. En effet elle va de 1,3 visites à KomkiIpala à 3 visites par femmes à Bassmyam. Or nous avions constaté plus haut que les agents DBC avaient visité en moyenne deux fois plus de femmes à Komki-Ipala qu'à Bassmyam (17,5 contre 34,4 femmes). On aurait pu en conclure hâtivement un plus grand dynamisme dans à Komki-Ipala. Or, en terme de nombre de visites reçues par les femmes, on arrive a la conclusion inverse.

Or les ADBC sont sensés aborder un nombre élevé de thèmes. Un ADBC ne peut aborder de façon approfondie plus de deux thèmes par visite. Il faudrait donc au moins 3 visites pour faire passer les messages. Une chose est de visiter un grand nombre de femmes, une autre est de leur consacrer le temps nécessaire pour esperer influer sur leurs attitudes.

Le Tableau 20 présente aussi le nombre moyen de visite par agent DBC (colonne de droite). Selon les FS, un agent DBC peut en moyenne effectuer entre 30 et 80 visites dans l'année. Si on tient compte des distances, des rendez vous manqué, etc, cela peut représenter jusqu'à une demi journée de travail par semaine, aux quels s'ajoutent les causeries. La charge de travail des ADBC est-elle raisonnable si on souhaite accroitre à la fois le nombre de femmes visitées et le nombre de visite par femmes ? Les simulations ci-dessous montrent que des choix s'imposent : soit on réduit le nombre de thèmes à aborder, soit on augmente le nombre d'ADBC, tout en augmentant par ailleur le rendement

Simulation 1. Pour 6 thèmes au total, 2 thèmes abordés par visite, un nombre maximal de 52 visites/ADBC/année (soit une visite par semaine) et pour couvrir 50\% des femmes de la Zone B, il faudrait 298 ADBC.

\begin{tabular}{|c|c|c|c|c|c|}
\hline \multirow[t]{2}{*}{ Formation sanitaire } & $\begin{array}{l}\text { Nombre de } \\
\text { femmes dans }\end{array}$ & Population & $\begin{array}{l}\text { Nbre de } \\
\text { visites }\end{array}$ & $\begin{array}{c}\text { DBC } \\
\text { necessaires }\end{array}$ & $\begin{array}{c}\text { Nbre actuel Ecart } \\
\text { de DBC }\end{array}$ \\
\hline & l'echantillon & & necessaires & & \\
\hline
\end{tabular}




\begin{tabular}{lcccccc} 
Bassmyam & 147 & 1838 & 2756 & 53 & 20 & -33 \\
Komki-Ipala & 140 & 1750 & 2625 & 50 & 8 & -42 \\
Komsilga & 228 & 2850 & 4275 & 82 & 23 & -59 \\
Lougsi & 78 & 975 & 1463 & 28 & 6 & -22 \\
Tintilou & 81 & 1013 & 1519 & 29 & 9 & -20 \\
Vipalogo & 153 & 1913 & 2869 & 55 & 18 & -37 \\
Total & 827 & 10338 & 15506 & 298 & 84 & -214 \\
\hline
\end{tabular}

Simulation 2. Pour 3 thèmes au total, 2 thèmes abordés par visite, un nombre maximal de 52 vistes/ADBC/année (une visite par semaine) et pour un taux de couverture de 50\%, il faudrait 149 ADBC soit moins de la moitié que si l'on devait traiter 6 thèmes.

\begin{tabular}{lcccccc}
\hline Formation sanitaire & $\begin{array}{c}\text { Nombre de } \\
\text { femmes dans } \\
\text { l'echantillon }\end{array}$ & Population & $\begin{array}{c}\text { Nbre de } \\
\text { visites } \\
\text { necessaires }\end{array}$ & $\begin{array}{c}\text { DBC } \\
\text { necessaires }\end{array}$ & $\begin{array}{c}\text { Nbre actuel Ecart } \\
\text { de DBC }\end{array}$ \\
\hline Bassmyam & 147 & 1838 & 1378 & 27 & 20 & -7 \\
Komki-Ipala & 140 & 1750 & 1313 & 25 & 8 & -17 \\
Komsilga & 228 & 2850 & 2138 & 41 & 23 & -18 \\
Lougsi & 78 & 975 & 731 & 14 & 6 & -8 \\
Tintilou & 81 & 1013 & 759 & 15 & 9 & -6 \\
Vipalogo & 153 & 1913 & 1434 & 28 & 18 & -10 \\
Total & 827 & 10338 & 7753 & 149 & 84 & -65 \\
\hline
\end{tabular}

Simulation 3. Pour 3 thèmes au total, 2 thèmes abordés par visite, un nombre maximal de 104 visites/ADBC/année (deux visites par semaine) et pour un taux de couverture de 60\%, 89 ADBC suffiraient!

\begin{tabular}{lcccccc}
\hline Formation sanitaire & $\begin{array}{c}\text { Nombre de } \\
\text { femmes dans } \\
\text { l'échantillon }\end{array}$ & Population & $\begin{array}{c}\text { Nbre de } \\
\text { visites } \\
\text { nécessaires }\end{array}$ & $\begin{array}{c}\text { DBC } \\
\text { nécessaires }\end{array}$ & $\begin{array}{c}\text { Nbre actuel } \\
\text { de DBC }\end{array}$ & Ecart \\
\hline Bassmyam & 147 & 1838 & 1654 & 16 & 20 & 4
\end{tabular}




\begin{tabular}{lcccccc} 
Komki-Ipala & 140 & 1750 & 1575 & 15 & 8 & -7 \\
Komsilga & 228 & 2850 & 2565 & 25 & 23 & -2 \\
Lougsi & 78 & 975 & 878 & 8 & 6 & -2 \\
Tintilou & 81 & 1013 & 911 & 9 & 9 & 0 \\
Vipalogo & 153 & 1913 & 1721 & 17 & 18 & 1 \\
Total & 827 & 10338 & 9304 & 89 & 84 & -5 \\
\hline
\end{tabular}

De ces trois séries d'hypothèses, on constate que le nombre de thèmes et le rendement jouent énormément sur les moyens à mettre en oeuvre pour esperer avoir un effet. Or les ADBC sont des bénévoles. Il n'est ni raisonnable, ni moral de leur exiger trop de sacrifices.

\subsubsection{Satisfaction des villageois (toutes zones)}

Les prestations effectives et les réalisations dépendent du soutien que la communauté accorde à la stratégie, qui lui-même dépend des perceptions des tout premiers bénéficiaires : ce sont eux qui ont contribué à faire la réputation des agents DBC et celle des FS. Nous voulons donc mesurer le degré de satisfaction exprimé par les enquêtés soumis à l'enquête de passage au sujet des différentes composantes de l'intervention.

\section{a. Les villageois sont-ils satisfaits des agents DBC (zone B) ?}

$1 \quad$ Les enquêtés sont-ils satisfaits du travail des agents DBC dans leur village (zone B) ?

Hypothèse d'évaluation : on suppose ici que les villageois ne soutiendront le programme que si celui-ci a bonne réputation, c'est-à-dire si les enquêtés expriment leur satisfaction. Dans la mesure où les réputations habituellement tendent plus à se détériorer qu'à s'améliorer, le degré de satisfaction après une année de mise en œuvre de l'intervention fournira un bon indicateur de ce qui peut être raisonnablement attendu dans les années à venir en termes de soutien des villageois. Ce point important sera analysé en détail dans la section 1.4

Résultats de l'évaluation.

L'évaluation du succès de la DBC passe aussi par la perception que la population a de l'intervention. Toutefois, le fait d'avoir entendu parler de la DBC, de connaitre l'ADBC ou encore de connaitre la finalité de l'intervention ne renseigne pas suffisamment sur leur perception vis à vis de la DBC. Or, la population ne peut soutenir le programme que si celui-ci a bonne réputation, c'est-à-dire que si la population exprime sa satisfaction. Le degré de satisfaction de la population fournit un bon indicateur de ce qui peut être 
raisonnablement attendu dans les années à venir en termes de soutien de la population aux activités des DBC. Pour approcher cet indicateur deux questions complémentaires ont été posées: (1) les enquêtés « ont confiance en l'intervention » et (2) «ils souhaitaient devenir ADBC ». Ainsi, sur les 448 enquêtées ayant entendu parler des ADBC, 335 (75\%) ont foi en l'intervention et 294 (66\%) souhaitent devenir un jour ADBC (cf 2). Si ces indicateurs semblent, a priori, satisfaisants, il faut être prudent quant à leur interprétation afin d'éviter toute satisfaction prématurée. Et ce pour deux raisons que nous developpons maintenant.

1) l'effet de nouveauté du programme peut expliquer cet engouement. Or l'enthousiasme des débuts peut s'effriter au fur et à mesure que la motivation des participants s'amenuise (d'ailleurs les ADBC se plaignent manque de revenus et de l'insuffisance de suivi de leur travail). De plus, la participation communautaire n'est clairement comprise que d'une minorité : sur 827 femmes, seulement 135 (16\%) ont entendu parler des CG. Il est à craindre donc que dans les années à venir les ADBC ne connaissent le même désintérêt si leur ardeur au travail continue à s'estomper.

2) Les mesures de l'exposition des femmes aux activités des ADBC (causeries, VAD...), on constate que les résultats sont très faibles. On a demandé aux femmes qui déclarent avoir reçu la visite de leur ADBC quel était l'objet de sa visite. Le tableau Tableau 21 présente les résultats. 
Tableau 21 : Couverture des différentes activités des ADBC.

\begin{tabular}{lcccr}
\hline & $\begin{array}{c}\text { Femmes connaissant le } \\
\text { programme DBC }\end{array}$ & \multicolumn{2}{c}{ Femmes visitées } \\
& $\mathrm{n}$ & $\%$ & $\mathrm{n}$ & $\%$ \\
\hline Nature de l'activité & & & & \\
CauseriesVAD/PF & 2213 & 39,5 & 1050 & 59,6 \\
Causeries VAD/MST-SIDA & 988 & 17,6 & 513 & 29,1 \\
Causeries VAD/Excision & 788 & 14,1 & 438 & 24,8 \\
Causeries VAD/Paludisme & 588 & 10,5 & 338 & 19,1 \\
Causeries VAD/Diarrhée & 200 & 3,6 & 113 & 6,4 \\
Causeries VAD/Vente SRO & 375 & 6,7 & 200 & 11,3 \\
Causeries VAD/Vente Contraceptifs & 713 & 12,7 & 275 & 15,6 \\
Causeries VAD/Vente chloroquine & 1300 & 23,2 & 475 & 27,0 \\
Nombre de femmes (N) & 5600 & 100,0 & 1763 & 100,0 \\
\hline
\end{tabular}

Nombre de femmes : $\mathrm{n}^{*} 1 /$ taux de sondage ; VAD : visite à domicile.

Source : LSC (UERD), enquête de passage 1998.

Ces résultats montrent que le nombre de concessions/ménages visités par les ADBC n'est pas suffisant pour conclure sur l'efficacité des ADBC. Une chose est d'être reçu dans une concession, une autre est d'y être écouté !

On peut se demander si l'éventail des thèmes n'est pas un handicap aussi bien pour l'ADBC que pour son interlocuteur. En effet, il est difficile d'aborder tous ces différents thèmes au cours d'une même visite. Cela nécessite du temps que la population n'est pas toujours prête à accorder. Il n'est pas acquis qu'elle accepte les visites répétées de l'ADBC (cf. section 1.2.22 page 72).

2 Tous les enquêtés souhaiteraient-ils devenir un agent DBC dans leur village (zone B) ?

Hypothèse d'évaluation. Le désir de devenir agent DBC constitue une autre manière de mesurer le soutien de la communauté pour la composante DBC. Si les agents DBC sont respectés et enviés, alors les autres villageois devraient exprimer leur souhait de devenir aussi un jour agent DBC.

\section{Résultat d'évaluation.}

Nous avons vu plus haut que parmi les 448 femmes enquêtées qui ont entendu parler de la DBC, 294 $(66 \%)$ souhaitent devenir un jour ADBC. Pourtant, seulement une minorité de ces femmes connaissent la finalité de la fonction, et peu d'entre elles ont déclaré avoir discuter de santé, de PF, etc. Il est donc 
probable que les femmes sont plus attirées par le prestige de la fonction (un ADBC n'est il pas assimilable à un logtor ?) et par la bicyclette que par le contenu du travail.

\section{b. Les villageois sont satisfaits de l'amélioration de leurs formations sanitaires ? (toutes zones)}

Par soucis d'économie, à la demande du Comité technique, les questions relatives aux perceptions des enquêtés sur les amelioration des FS dans les Zones A et B n'ont pas été incluses dans le questionnaire de l'enquête de passage. 


\subsection{L'intervention a-t-elle eu un effet?}

Nous formulons à nouveau les objectifs de l'intervention sous forme de questions simplifiées. En fournissant des réponses à chacune de ces questions, l'équipe de recherche fournira les résultats atteints par l'intervention ainsi que son impact (ou indiquera les aspects où l'effet attendu ne s'est pas produit).

\subsubsection{Réduction de la fécondité}

\section{a. Le niveau de fécondité a-t-il significativement baissé entre l'enquête de base et les autres passages ?}

$1 \quad$ La réduction de la fécondité est-elle plus forte dans les zones d'intervention?

Nous ne nous attendions pas à observer une réduction significative de la fécondité dans aucune des quatre zones avant l'an 2000 dans la mesure où il faut une période relativement longue, au moins cinq à six ans après le démarrage d'une intervention en PF pour observer des changements structurels dans les comportements.

L'indice synthétique de fécondité (ISF) n'a pas connu de variation sensible au cours de la période même dans les zones d'intervention. En effet, l'ISF s'établit à 6,7 et 6,4 enfants par femme respectivement en 1996 et 1998 pour l'ensemble du champ d'investigation. En ce qui concerne les zones d'intervention A et B, celles-ci enregistrent pour 1996 et 1998 respectivement 6,8 et 6,6 enfants par femme dans la zone A et 6,3 et 6,1 dans la zone B. Nous avons constater que la couverture de l'intervention est à la fois faible et superficielle. Les variations de la fécondité observé sont donc plus conjoncturelles que le résultat de l'intervention des DBC.

\section{b. Par quels mécanismes cette réduction s'est-elle opérée (ou pourrait-elle s'opérer à l'avenir) ?}

Ce thème mérite d'être étudié même en l'absence d'une baisse de la fécondité. En effet, une augmentation dans la demande de contraceptifs ou dans leur utilisation indiquerait un changement de comportements annonciateur d'une baisse dans un futur plus lointain. 
1 La prévalence contraceptive a-t-elle plus augmenté dans les zones B et/ou A que dans la zone C?

Nous considérons pour les femmes âgées de 15 à 49 en union en 1996 et en 1998 la variable «Utilisation actuelle d'une méthode contraceptive moderne» En raison de la faiblesse des effectifs de femmes utilisant chaque méthode contraceptive moderne, la présentation est faite non pas par méthode contraceptive mais pour l'ensemble des méthodes contraceptives modernes (le nombre total par zone des femmes utilisant une méthode contraceptive moderne ne dépasse jamais 40). Le Tableau 22 qui suit indique pour chaque zone, la prévalence contraceptive en 1996 et 1998.

Tableau 22 : Proportion de femmes mariées utilisant une méthode de PF moderne.

\begin{tabular}{lcccccccr}
\hline & Zone A & \multicolumn{2}{c}{ Zone B } & \multicolumn{2}{c}{ Zone C } & \multicolumn{2}{c}{ Zone D } \\
& 1996 & 1998 & 1996 & 1998 & 1996 & 1998 & 1996 & 1998 \\
\hline Utilise une methode & \multicolumn{1}{c}{} & & & & & & \\
moderne (\%) & 3,7 & 5,9 & 3,6 & 8,0 & 3,2 & 2,7 & 7,4 & 7,3 \\
$\mathrm{~N}$ & 590 & 496 & 529 & 488 & 687 & 664 & 527 & 438 \\
\hline \multicolumn{2}{l}{ Source : LSC (UERD), enquête de base 1996 et enquête de passage 1998. }
\end{tabular}

La prévalence contraceptive a augmenté dans la zone A (de 60\%, P=0,1) En zone B, le taux de prévalence a plus que doublé (augmentation de $122 \%, \mathrm{P}<0,05$ ). Au cours de la même période, les taux de prévalence sont restés quasi stationnaires dans les zones $\mathrm{C}$ et $\mathrm{D}$. 
Figure 11 : Evolution de la prévalence contraceptive entre 1996 et 1998, par zone.

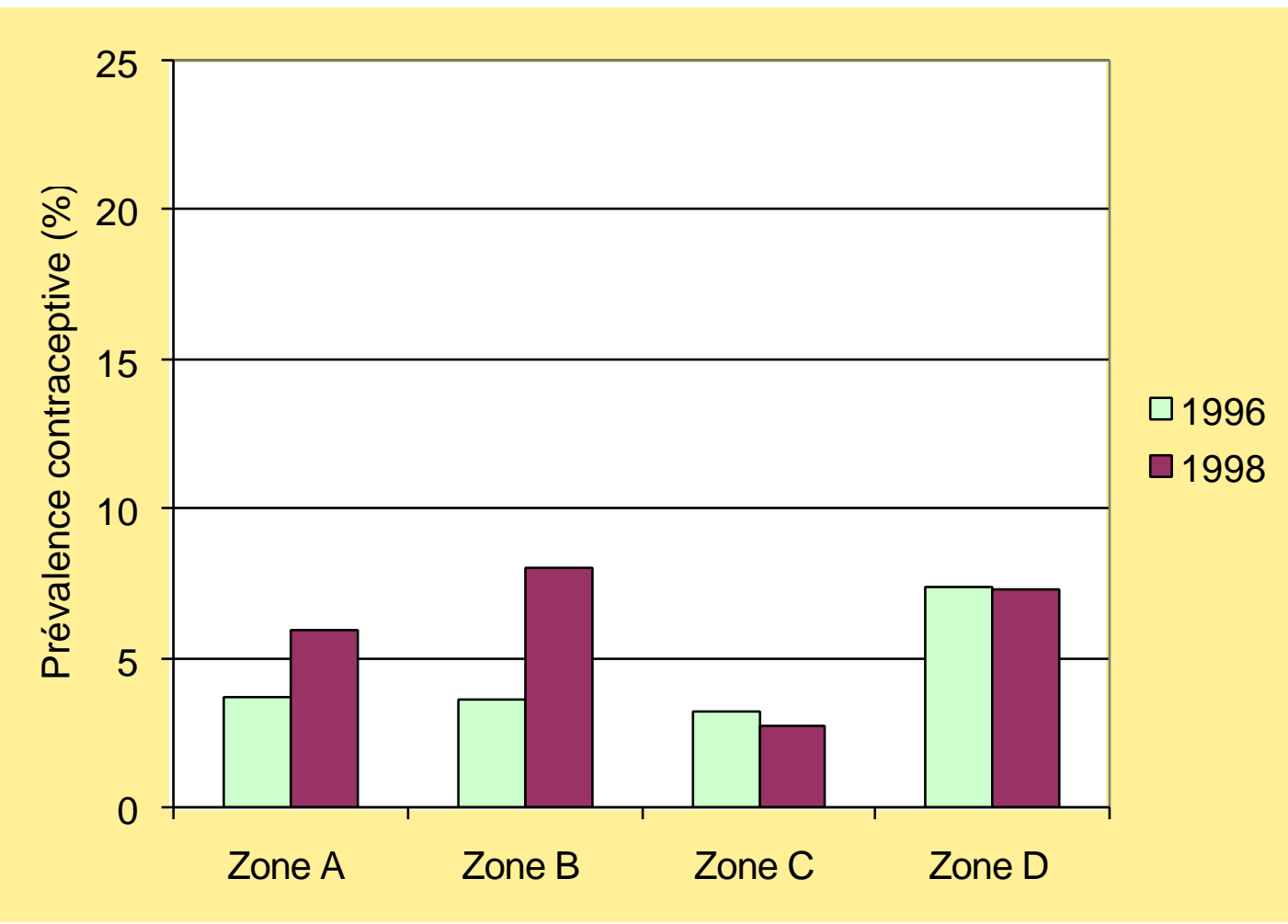

Source : LSC (UERD), enquête de base 1996 et enquête de passage 1998.

2 La demande contraceptive a-t-elle plus augmenté dans les zones B et/ou A que dans la zone C $?$

La demande contraceptive est appréciée en prenant en compte l'utilisation actuelle d'une méthode contraceptive et l'intention d'en utiliser une à l'avenir. Le tableau 3..2 qui suit se limite aux intentions d'utilisation à l'avenir de méthodes contraceptives. 
Tableau 23 : Intention d'utiliser une méthode contraceptive moderne par zone en 1996 et 1998.

\begin{tabular}{lcccccccc}
\hline & \multicolumn{2}{c}{ Zone A } & \multicolumn{2}{c}{ Zone B } & \multicolumn{2}{c}{ Zone C } & \multicolumn{2}{c}{ Zone D } \\
& 1996 & 1998 & 1996 & 1998 & 1996 & 1998 & 1996 & 1998 \\
\hline $\begin{array}{l}\text { Compte utiliser à } \\
\text { l'avenir (\%) }\end{array}$ & 29,8 & 64,9 & 33,3 & 65,8 & 39,0 & 50,2 & 34,2 & 64,8 \\
$\begin{array}{l}\text { Compte utiliser les 12 } \\
\text { prochain mois (\%) }\end{array}$ & 12,9 & 43,9 & 24,2 & 48,8 & 24,5 & 36,8 & 21,4 & 46,4 \\
\hline
\end{tabular}

Source : LSC (UERD), enquête de base 1996 et enquête de passage 1998.

L'analyse des intentions d'utilisation de méthodes contraceptives dans l'avenir indique des taux nettement plus élevés que les taux d'utilisation effective, et ce quelque soit la zone. Il y aurait donc une demande encore insatisfaite de contraception

Dans toutes les zones, l'augmentation des intentions d'utilisation à l'avenir est forte. Le taux a été multiplié par 2,2 en zone A; 2 en zone B; 1,9 en D et 1,3 en C. Que l'avenir soit ou non précisé, la zone témoin (Zone C) est celle où l'augmentation des intentions d'utilisation est relativement plus faible qu' ailleurs.

La forte augmentation des intentions d'utilisation des méthodes contraceptives entre 1996 et 1998 peut être attribuée, au moins en partie à l'enquête panel elle-même. En effet les enquêteurs constituent une source d'information sur la PF pour les populations qu'ils ont visitées, comme l'attestent d'ailleurs en 1998 certaines réponses d'enquêtées à la question relative à la source d'information sur la PF.

A noter également que les intentions restent beaucoup plus fortes lorsque l'avenir n'est pas circonscrit. Lorsque l'avenir est réduit aux 12 prochains mois, le taux d'intention baisse parfois de moitié. Cela confirme ce qui a été dit plus haut : es femmes sont le susceptible d'utiliser une méthode contraceptive moderne qu'une fois qu 'elles arrive à un certain âge ou a une certaine parité.

Aux âges plus jeunes, le recours aux méthodes contraceptives est fait davantage dans une optique d'espacement des naissances plutôt que de limitation de celles-ci. On comprend donc que dans le proche avenir, opérationalisé par les douze prochains mois, certaines utilisatrices potentielles souhaitent tomber enceintes tout en envisageant de recourir à la contraception plus tard.

Si la prévalence contraceptive a augmenté de façon significative dans la zone d'intervention par excellence (zone B) comparativement aux autres zones, la demande contraceptive ne semble pas quant à elle avoir subi la même tendance. Le niveau de la demande contraceptive enregistrée en 1998 est sensiblement le même dans les zones A et B. Il apparait donc que les agents DBC ne semblent pas être parvenus à susciter 
un besoin nouveau de contraception au sein des populations qu'ils avaient à charge de sensibiliser à la PF. Ils n’ont pu que mettre à la disposition des femmes qui étaient déjà disposées à adopter une méthode contraceptive, les moyens de satisfaire leur aspiration

Comme indiqué à la question Q3.1-a1, on n'observe pas de variation significative de la fécondité entre 1996 à 1998 dans les zones d'intervention.. La durée de l'intervention est trop courte (18) mois et la croissance de la prévalence contraceptive trop faible pour pouvoir infléchir le niveau de la fécondité.

\section{c. L'intervention a-t-elle eu des effets durables ?}

Cela est difficile à évaluer à priori. Au mieux, nous pourrions supposer qu'un effet est durable si les changements décrits au thème 1.2.1b sont suffisamment importants pour marquer une rupture dans les comportements ou renverser une tendance.

$1 \quad$ Y a t-il une augmentation de l'utilisation de la contraception chez les jeunes gens ?

Les jeunes présentent un intérêt particulier dans la mesure où du comportement reproductif de cette catégorie de la population, dépendra en grande partie l'évolution future de la fécondité.

Pour pouvoir analyser l'utilisation de la contraception chez les jeunes, nous avons aggregé les deux premiers groupes d'âge (15-24). Il ressort que la prévalence contraceptive est légèrement plus faible au sein de la population (féminine) jeune (3,6 et 4,9\% respectivement en 1996 et 1998) comparativement à l'ensemble des femmes en âge de procréer (4,4 et 5,4\% respectivement en 1996 et 1998)

L'examen de la prévalence contraceptive par zone indique toutefois une hausse significative $(\mathrm{p}<0,05)$ dans les zones A et B au cours de la période 1996-1998. En 1998 dans la zone A, la prévalence contraceptive chez les jeunes femmes est légèrement supérieure à celle de l'ensemble des femmes en âge de procréer. Tout comme pour l'ensemble des femmes en âge de procréer, la présence des agents DBC dans la zone B semble influencer positivement la prévalence contraceptive chez les jeunes si l'on en juge par la réduction de l'écart de prévalence entre les zones A et B, de 1996 (écart de 1 à 2,6) à 1998 (écart de 1 à 1,5). 
Tableau 24 : Prévalence contraceptive chez les jeunes femmes (15-24 ans) à ceux de l'ensemble des femmes en union, par zone en 1996 et 1998.

\begin{tabular}{lcccccccc}
\hline & Zone A & \multicolumn{2}{c}{ Zone B } & \multicolumn{2}{c}{ Zone C } & \multicolumn{2}{c}{ Zone D } \\
& 1996 & 1998 & 1996 & 1998 & 1996 & 1998 & 1996 & 1998 \\
\hline $\begin{array}{l}\text { Utilise methode } \\
\text { moderne }\end{array}$ & & & & & & & & \\
ensemble (15-49 ans) & 3,7 & 5,9 & 3,6 & 8,0 & 3,2 & 2,7 & 7,4 & 7,3 \\
jeunes (15-24 ans) & 3,1 & 7,0 & 1,2 & 4,6 & 4,3 & 2,5 & 5,9 & 6,1 \\
\hline
\end{tabular}

Source : LSC (UERD), enquête de base 1996 et enquête de passage 1998.

$2 \quad$ Y a t-il eu des changements favorables dans les perceptions de la PF ?

La perception de la PF par les populations a été appréhendée à partir des réponses à la question relative à l'existence ou non d'inconvénients associés aux méthodes contraceptives. Il en ressort que la proportion des femmes qui déclarent des inconvénients associés à l'utilisation de méthodes contraceptives est faible (moins de 5\%). Il importe cependant de relever que les femmes qui déclarent ne pas savoir représentent une proportion non négligeable, autour de 16\% (15\% en 1996 et 17\% en 1998). Celles-ci ne sont pas, selon toute vraisemblance, prêtes à adopter une méthode contraceptive. Dans le doute, elles préfereront s'en abstenir. En prenant en compte ces deux catégories, c'est environ une femme sur 5 qui a une perception défavorable de la PF en 1996 et en 1998.

Pour la zone B, la persistance (aux deux dates) d'une proportion de plus de $10 \%$ de femmes sans opinion sur l'existence éventuelle d'inconvénients associés aux méthodes contraceptives remettrait en cause l'efficacité du travail des agents DBC mis à contribution dans ladite zone.

$3 \quad$ Y a t-il une réduction de la mortalité infantile ?

La taille des échantillons par zone est trop petite pour obtenir des taux de mortalité valide. Les différences sont principalement due à l'aléas.

Tableau 25 : taux de mortalité par zone et par année.

\begin{tabular}{lcrrrrrrr}
\hline & \multicolumn{2}{c}{ Zone A } & \multicolumn{2}{c}{ Zone B } & \multicolumn{2}{c}{ Zone C } & \multicolumn{2}{c}{ Zone D } \\
& 1996 & 1998 & 1996 & 1998 & 1996 & 1998 & 1996 & 1998 \\
\hline mortalité infantile & 114 & 80 & 109 & 80 & 140 & 134 & 94 & 55 \\
mort infanto-juvénile & 178 & 159 & 151 & 129 & 209 & 198 & 225 & 129 \\
\hline
\end{tabular}

Source : LSC (UERD), enquête de base 1996 et enquête de passage 1998. 


\subsection{Quelle est la stratégie d'intervention la plus pertinente?}

\subsubsection{Quel est l'impact relatif des différentes approches sur la demande de contraception/PF ?}

1 L'augmentation de la demande de contraception dans la zone B est-elle supérieure à celle de la zone $\mathrm{A}$, elle-même supérieure à celle de la zone $\mathrm{C}$ ?

Comme indiqué à la question 1.1.1b.2, on utilise la définition de l'EDS de la demande potentielle de contraception (cf. définition page 44). Rappelons juste qu'il s'agit en fait de besoins «objectifs » (selon les hypothèses de l'intervention) et non d'une demande exprimée par les femmes. On constate que ces besoins restent inchangés entre 1996 et 1998, ce qui n'est pas étonnant : on ne peut pas s'attendre à ce que les intentions en matière de fécondité évoluent en si peu de temps.

Figure 12 : Evolution de la demande potentielle de contraception entre 1996 et 1998, par zone.

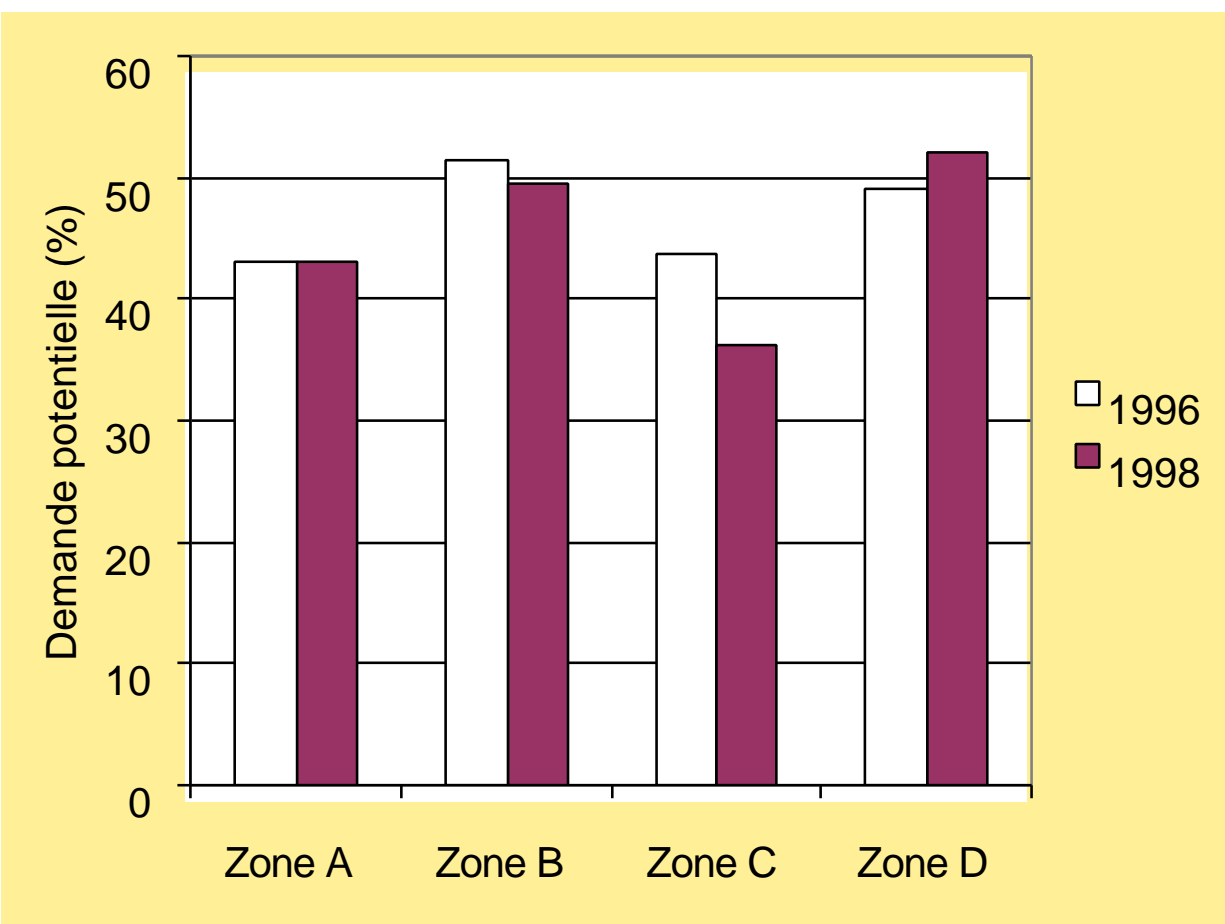

Source : LSC (UERD), enquête de base 1996 et enquête de passage 1998. 
Les perceptions sur la PF ont-elles changé plus favorablement dans la zone B que dans la zone A et en zone A que dans la zone C ?

A la question Q3.1-c2, nous avons utilisé la proportion des femmes qui déclarent que les méthodes contraceptives comportent des inconvénients pour apprécier l'évolution des perceptions sur la PF. Une autre variable permettant d'évaluer les perceptions est la proportion des femmes qui déclarent qu'elles accepteraient les méthodes contraceptives si elles étaient gratuites. Cette seconde variable n'a été recueillie qu'en 1998. Les résultats indiquent qu'environ une femme sur trois n'accepterait pas les produits contraceptifs en dépit de leur gratuité alors qu'elles ont un besoin non satisfait de PF (definition de l'EDS, cf. page 44). La comparaison entre zones (Figure 13) ne révèle aucune différence significative. Ce résultat va dans le même sens que d'autres mentionnés précédemment et qui indiquent des insuffisances dans l'activité de sensibilisation des populations (de la zone B) autour de la PF par les agents DBC. Notons que les réponses à ce type de question n'est pas exempt de complaisances. Qui refuserait un cadeau?

Figure 13 : Proportion de femmes mariées ayant un besoin non satisfait de PF et qui déclarent accepter une méthode contraceptive si celle-ci était gratuite.

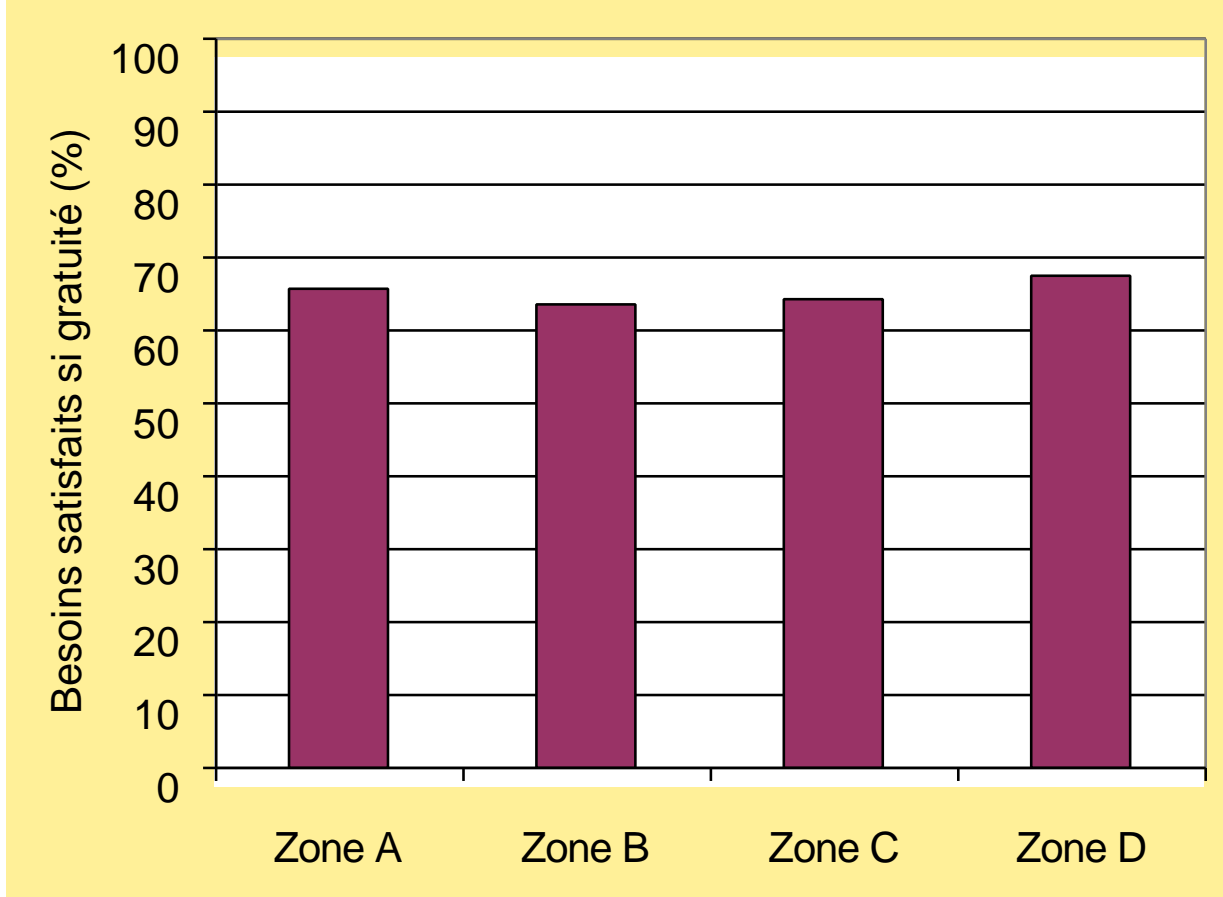

Source : LSC (UERD), enquête de base 1996 et enquête de passage 1998. 
L'analyse selon l'âge indique que les jeunes femmes (âgées de moins de 20 ans) mais surtout les femmes âgées de 40 ans et au-delà sont celles où le taux de perception défavorable est le plus élevé (Figure 14). Les moins de 20 ans ont besoin de fonder une famille alors que les plus de 40 ans s'acheminent vers la ménopause.

Figure 14 : Proportion des femmes qui n'accepteraient pas de contraceptifs gratuits selon l'âge et la zone (1998).

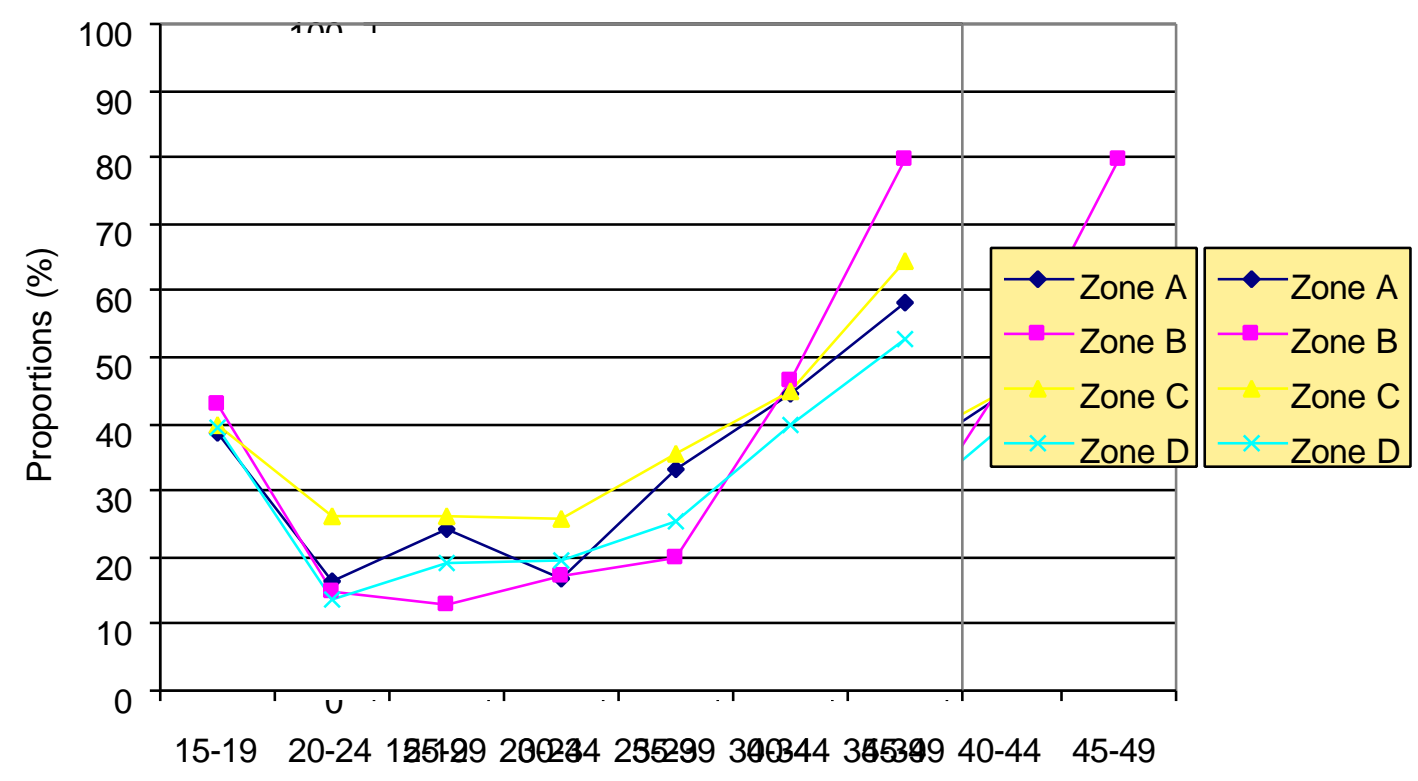

Source : LSC (UERD), enquête de base 1996 et enquête de passage 1998.

3 La variation de la demande satisfaite pour la contraception sont-elles différentes entre les zones $\mathrm{A}, \mathrm{B}$ et $\mathrm{C}$ ?

La demande contraceptive satisfaite est évaluée en rapportant l'utilisation effective de méthodes contraceptives modernes à la demande potentielle en PF (Figure 15). 
Figure 15 : Evolution de la demande satisfaite de contraception entre 1996 et 1998, par zone.

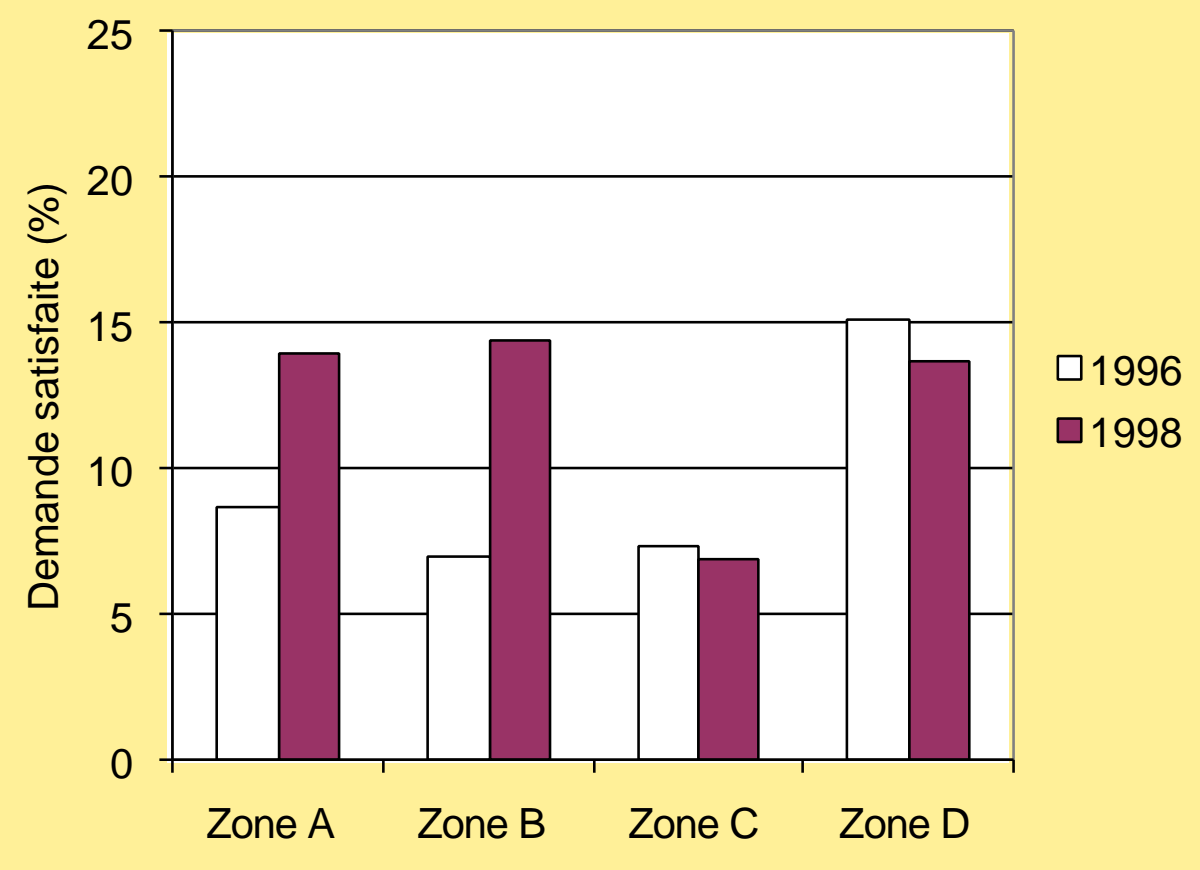

Source : LSC (UERD), enquête de base 1996 et enquête de passage 1998.

Les résultats indiquent une forte proportion de demande non satisfaite en matière de contraception. Celle-ci s'établit autour de 40\%. Dans aucune des zones, y compris la zone d'intervention par excellence (zone B) le niveau de la demande non satisfaite n'a connu une variation significative entre 1996 et 1998; montrant ainsi que la portée de l'activité DBC demeure manifestement modeste.

\subsubsection{L'approche communautaire est-elle plus efficace qu'une distribution basée dans les formations sanitaires?}

La stratégie DBC ne semble pas recueillir toutes les caractéristiques requises pour répondre au qualificatif de participation communautaire (voir annexe pour plus de détails). Par conséquent, nous analyserons seulement l'effet de la présence de l'agent DBC sur l'utilisation de la contraception et non l'ensemble du processus de participation communautaire.

1 La présence des agents DBC est-elle associée à une augmentation de la connaissance des méthodes contraceptives? 
Tableau 26 : Proportion de femmes mariées ayant entendu parler de contraception, 1996 et 1998.

\begin{tabular}{lcccccccc}
\hline & \multicolumn{1}{c}{ zone $\mathrm{A}$} & \multicolumn{2}{c}{ zone B } & \multicolumn{2}{c}{ zone C } & \multicolumn{2}{c}{ zone D } \\
& 1996 & 1998 & 1996 & 1998 & 1996 & 1998 & 1996 & 1998 \\
\hline \% de femmes mariées qui ont entendu & & & & & & & & \\
parler de: & & & & & & & & \\
-méthode moderne & 45,6 & 58,4 & 57,7 & 65,2 & 53,9 & 47,9 & 65,8 & 68,8 \\
-pilule & 32,7 & 35,3 & 29,3 & 30,7 & 30,0 & 29,7 & 13,5 & 31,8 \\
-DIU & 10,8 & 18,1 & 27,0 & 28,7 & 21,5 & 19,1 & 26,9 & 28,5 \\
-injections & 28,8 & 36,5 & 36,1 & 38,2 & 39,3 & 27,1 & 42,9 & 45,8 \\
-condoms & 44,4 & 58,4 & 51,2 & 56,1 & 44,4 & 42,3 & 43,1 & 58,4 \\
\hline
\end{tabular}

Source : LSC (UERD), enquête de base 1996 et enquête de passage 1998.

La proportion de femmes ayant entendu parler de méthodes modernes de contraception a cru de façon substantielle uniquement dans le Zone A (injections, DIU et condoms) et dans la Zone D (pilule et condom)zones, a l'exception de la zone C. Ces données n'indique pas un effet DBC au niveau de la connaissance de méthode.

2 La présence des agents DBC est-elle associée à une augmentation de la prévalence de l'utilisation des méthodes contraceptives?

Oui (cf. 1.3.1b.1), mais de façon modeste.

\subsubsection{L'approche communautaire est-elle meilleure en termes de coût-efficacité ?}

\section{a. Est-ce que la formule payante de recouvrement des coûts constitue une entrave à l'utilisation de la contraception?}

$1 \quad$ Quel est le coût du programme ?

L'UERD n'est pas en sa possession les éléments permettant de répondre aux questions relatives aux coûts du programme. La DSF completera en fonction de ses données comptables.

2 Quel est le coût de l'intervention par zone ?

Cf. remarque précédente.

3 Combien les enquêtées déclarent-elles être prêtes à payer pour des contraceptifs ? (voir aussi Q1.1-d2)

On demande aux enquêtées qui ont l'intention d'utiliser un contraceptif donné quel prix elles sont prêtes à payer pour s'approvisionner pendant un an. Nous nous attendons à obtenir un montant 
bas et nous l'interpréterons comme un minimum, le maximum étant le prix du contraceptif sur le marché. (voir aussi Q1.1-d2)

4 Est-ce que le prix des contraceptifs est un facteur de poids dans la décision de recourir à la contraception?

Non. Le coût des contraceptifs, quelque soit leur méthode, est faible, surtout si l'on on le compare au coût d'un enfant (nourriture, soins, etc.). Il est peu probable qu'une diminution supplémentaire du prix des contraceptifs accroissent leur utilisation.

$5 \quad$ Les méthodes contraceptives moins chères sont-elles préférées aux méthodes plus onéreuses ?

Non.

$6 \quad$ Les enquêtés font-ils confiance aux agents DBC et aux comités de gestion?

Il est improbable que les villageois soutiennent les efforts des agents DBC et acceptent de payer des contraceptifs onéreux s'ils soupçonnent l'agent DBC ou le comité de gestion de mauvaise gestion ou de malversation. On se reportera a la quatrième partie pour la réponse à cette question.

7 La formule de recouvrement des coûts par les agents DBC est-elle plus efficace ?

Le coût de la mise en ouvre et de la gestion d'une formule de recouvrement des coûts peut dépasser ses bénéfices. Dans ce cas, il pourrait être recommandé de proposer d'autres façons de désintéresser les agents DBC plutôt que de leur donner une ristourne (commission) sur la vente de contraceptifs.

L'UERD n'est pas en sa possession les éléments permettant de mesurer le coût que représente l'encadrement des DBC. Par ailleurs, on a que cet encadrement souffre de sérieuses lacune. L'estimation du coût de cet encadrement que fournira la DSF en fonction de ses données comptables sous-estimera probablement les moyens qui doivent être mobiliser pour assurer une un encadrement efficace de la participation communautaire (cf. recommandations). 


\section{b. Les enquêtés (es) préfèrent-ils (elles) s'approvisionner en contraceptifs auprès des agents DBC de leur village plutôt qu’à leur formation sanitaire ?}

1 Y a t-il des individus qui connaissent l'agent $\mathrm{DBC}$ de leur village et qui continuent à s'approvisionner à la FS ?

2 Y a t-il des individus qui connaissent l'agent $\mathrm{DBC}$ de leur village et qui continuent à s'approvisionner ailleurs?

3 Y a t-il des enquêtés qui affirment vouloir utiliser des méthodes contraceptives modernes mais qui ne savent pas où s'approvisionner?

\subsubsection{L'approche communautaire est-elle plus durable?}

La durabilité est un concept difficile à saisir. Elle ne peut vraiment être appréciée qu'après la fin de l'expérimentation, c'est-à-dire au bout de cinq ou dix ans de mise en œuvre du programme, une fois l'enthousiasme des premières années retombé. En outre, c'est la durabilité de l'impact du programme qui est visée et non la durabilité des institutions. Puisque l'institution chargée de la mise en œuvre est le gouvernement burkinabe, il est raisonnable d'affirmer que la durabilité institutionnelle peut être atteinte. Dans la zone $\mathrm{B}$, elle exige en plus le soutien à long terme des villageois.

La durabilité du soutien accordé par les villageois dépend d'un certain nombre de conditions nécessaires (mais non suffisantes). Les plus importantes de ces conditions sont les suivantes :

- Le programme pour lequel le soutien des villageois est recherché répond de manière satisfaisante à un besoin perçu comme une priorité par ceux-ci. Les populations considèrent que ce besoin concerne la communauté (c'est-à-dire qu'il n'est pas d'ordre personnel ou privé)

- Une proportion significative de la population comprend, croit et adhère à la démarche communautaire qui implique un de leur représentant associé au comité de gestion. Les ressources que les villageois contribuent au programme sont bien gérées. Les superviseurs et responsables de la gestion sont fiables et inspirent la confiance. Leur mandat est clairement défini.

- Les représentants des villageois et les agents DBC sont motivés par leur travail.

- Une proportion significative des villageois bénéficie de l'intervention et est satisfaite des prestations offertes. La satisfaction dépend de l'accessibilité, du coût et de la qualité de ces prestations (ce thème est abordé dans la section 1.3.3). 
- Une institution gouvernementale (ou une ONG solide) soutient activement les efforts des villageois. Cette institution étant le ministère de la santé, cette dernière condition devrait être acquise.

Nous reprendrons point par point le niveau de réalisation de ces six conditions.

1. La planification familiale doit être prioritaire dans la communauté : nous nous appuierons sur les entretiens de groupe réalisés à Bangma et à Nabele pour étayer nos analyses. Si cela peut être considéré comme un nombre infime de discussions au regard du nombre de villages couverts par l'expérimentation, notre connaissance du terrain et nos précédentes enquêtes dans la même zone d'enquête où nous avons réalisés 39 entretiens de groupe sur la reproduction nous permettent de poser quelques conclusions.

Nous pouvons dire que les besoins prioritaires énumérés par les populations sont d'abord des besoins en infrastructures : puits, route, barrage, école, dispensaire. Au niveau de la santé, les problèmes prioritaires à résoudre sont de traiter le paludisme, le rhume et la toux, les épidémies comme la rougeole et la méningite qui ont sévi de manière intense ces dernières années. La planification familiale, l'espacement des naissances ou le recours à la contraception apparaissent comme des affaires privées dans lesquelles la communauté ne peut rien recommander. Si le recueil des normes et valeurs dans les discours (Ouedraogo, 1997) confirme que les familles reconnaissent qu'avoir un grand nombre d'enfants comporte de nombreux inconvénients, il n'est cependant pas encore admis de vouloir interrompre l'ordre naturel des choses, d'autant plus qu'un grand nombre d'enfants présente des avantages non négligeables.

* Au regard de ce que nous avons trouvé, il n'apparait pas que la planification familiale soit prioritaire dans la communauté.

2. La communauté doit adhérer à la démarche : les populations sont difficiles à mobiliser pour les causeries à tel point que certains agents DBC profitent des réunions organisées par l'agent d'encadrement agricole pour délivrer quelques messages de sensibilisation sur la contraception. La communauté n'adhère donc pas à la démarche.

3. La communauté doit soutenir la stratégie : elle fait confiance aux agents, s'est appropriée l'intervention, et y participe.

* Les agents ont la confiance de la communauté dans la mesure où c'est celle-ci qui les a délibérément choisis après avoir été informée de leur rôle. Nous pouvons dire que la communauté ne s'est pas appropriée cette stratégie dans la mesure où pour les raisons évoquées précédemment, avoir des enfants relèvent de choix privés. La vente des contraceptifs est très réduite. Par contre les populations 
s'approvisionnent en aspirine et chloroquine auprès des agents DBC qui servent de référence pour les maux de tête.

* La communauté ne s'est pas appropriée l'intervention.

4. La communauté doit être satisfaite des prestations offertes en termes de coûts, d'accessibilité et de qualité des prestations.

* coût : Bien que le prix puisse paraître modique, en période de soudure il est prohibitif. La quasi totalité des clientes des agents DBC ont interrompu la consommation de pilules en raison de leurs moyens insuffisants. Certaines se sont approvisionnées à crédit. De plus il y a une forte concurrence avec le marché car les produits se retrouvent presque au même prix sur le marché qui présente l'avantage de plus de discrétion, même s'il y a plus de risques sur la qualité des produits (péremption).

* L'accessibilité comporte plusieurs dimensions. Outre l'accessibilité économique ou le coût qui peut constituer un obstacle, l'accessibilité physique en terme de distance peut représenter un facteur favorisant ou non. Dans le cas de la stratégie à base de distribution communautaire, les agents issus de la communauté et choisis par celle-ci représente sans aucun doute un facteur favorisant le rapprochement des services et des prestations des bénéficiaires. Le constat est que la proximité de l'aspirine et la nivaquine est particulièrement apprécié des villageois. En matière de contraceptifs, certaines clientes préfèrent aller hors du village pour s'approvisionner par souci de discrétion. L'accessibilité culturelle est moins évidente car certaines populations refusent catégoriquement de songer à espacer les naissances en raison de leurs convictions religieuses. Ce serait pécher contre Dieu que d'intervenir dans un processus naturel. C'est ainsi que certains quartiers protestants ou musulmans sont hostiles à tout message. La sensibilisation à la contraception se heurte alors à des valeurs très fortement ancrées et difficiles à ébranler. Néanmoins, certains individus de ces communautés ont recours à la contraception, à l'insu du groupe. Lorsque cela ne peut se cacher, on recourt le plus souvent à des arguments tels que ceux de santé défaillante de la mère pour justifier de pratiques innovatrices qui auraient par ailleurs la réprobation du groupe. Ces comportements actuellement considérés comme déviants sont précurseurs de changements plus importants à long terme.

* La qualité des prestations peut être appréhendée tant du côté des services délivrés par les agents que de celui des produits offerts. On peut la qualifier de douteuse car certains produits sont périmés (cas de l'aspirine) ou absents par épuisement des stocks et non approvisionnement (cas de la pilule).

* La communauté n'est pas totalement satisfaite des prestations.

5. Les agents DBC doivent être motivés : les formes de motivation ou d'encouragement peuvent être diverses. Si la forme la plus courante est la motivation matérielle, il n'en demeure pas moins qu'elle ne 
suffit pas pour assurer la pérennité de la stratégie : elle doit s'accompagner d'autres formes de motivations qui doivent constituer des incitations morales.

* S'agissant de la motivation matérielle, elle consiste d'une part en une ristourne sur les produits, d'autre part d'une sacoche renfermant la première dotation de produits et d'une bicyclette. La ristourne sur les produits peut être motivante à condition que les produits se vendent bien. Il s'avère que cette ristourne sur les contraceptifs n'est pas même suffisante pour entretenir la bicyclette : elle oscille entre 100 et $1500 \mathrm{~F}$ par mois, selon les déclarations des agents. La ristourne sur l'aspirine et la nivaquine n'est pas encore connue, ce qui ne constitue pas un sujet d'encouragement alors que cela pourrait l'être : en effet, ces produits s'écoulent à un rythme plus rapide et la demande est importante. La bicyclette constitue sans aucun doute un sujet de motivation, tout au moins au regard des commentaires des populations. L'agent DBC s'identifie facilement par la bicyclette et la sacoche et est reconnu comme une personne s'occupant de la santé, parfois encore appelés « docteurs ». Les anciens agents PSP ou agents de santé communautaire avaient d'ailleurs déjà une bicyclette du fait de leur précédente fonction : ils se retrouvent ainsi avec deux bicyclettes ce qui crée parfois des sentiments d'envie et de jalousie de la part des accoucheuses villageoises qui se déplacent à pieds.

* Les aspects non matériels de la motivation sont malheureusement insuffisants voire insignifiants pour compenser le peu de satisfaction matérielle liée à la ristourne. En effet, la supervision du personnel de santé est irrégulière, la formation continue inexistante ainsi que les rencontres entre agents pour échanges d'expérience. Un recyclage régulier permettrait d'encourager fortement les agents dans leurs activités ainsi que cela a pu être observé à la suite des formations spécifiques sur l'excision et les maladies diarrhéiques qui ont permis un regain d'activités.

* Il n’y a donc pas de motivation suffisante pour que la stratégie soit durable.

6. L'institution de tutelle doit accorder son soutien à l'intervention : ce soutien devrait se traduire par une présence effective et régulière sur le terrain, sous forme de supervision, d'approvisionnement en produits et de formation. Si cela semble aller de soit comme nous l'avions mentionné dans le protocole d'évaluation, la réalité observée est loin de donner satisfaction.

* Tous les agents DBC n'ont pas reçu de supervision «à domicile » des personnels des formations sanitaires, malgré la dotation en carburant et en moyen de déplacement. Dans une formation sanitaire où le responsable a été affecté, cette supervision n'a pas eu lieu depuis six mois. Le personnel des formations sanitaires se sent abandonné de ses supérieurs hiérarchiques car ils ne reçoivent pas l'appui qu'ils étaient en mesure d'attendre du district. Il n'y a pas eu de recyclage ni de visites d'échanges ainsi que cela avait été prévu. 
* La plupart des personnels ont été affectés et les nouveaux n’ont pas reçu de formation.

L'approvisionnement en produits est défaillant et le circuit parfois incohérent : la formation sanitaire de Vipalogo doit s'approvisionner à Sapone alors qu'il n’y a pas de route.

* C'est à ce niveau que se trouve le maillon le plus faible de la stratégie et qui mériterait d'être renforcé.

Le Tableau 27 résume les conditions de durabilité réunies après 18 mois d'intervention

Tableau 27 : conditions de durabilité de la stratégie DBC.

\begin{tabular}{ll}
\hline Conditions requises & Réunies \\
\hline$-\quad$ PF prioritaire pour la communauté & Non \\
$-\quad$ Adhésion à la démarche & Non \\
$-\quad$ Motivation des DBC & Oui et non \\
$-\quad$ Satisfaction des villageois & Oui et non \\
Accessibilité & Oui et non \\
Coût & Non \\
Qualité prestations & Non \\
$-\quad$ Soutien de la communauté & Non \\
$-\quad$ Soutien institutionnel & Non \\
\hline
\end{tabular}

\section{a. Les villageois perçoivent-ils la maîtrise de leur fécondité comme un besoin urgent et prioritaire qui doit être résolu par la collectivité ?}

La question de la planification familiale est une affaire privée et non collective. Ce n'est pas une question de santé prioritaire au regard des maladies courantes auxquelles ont à faire face les populations. Ce sont d'abord les médicaments tels que l'aspirine et la nivaquine que l'on cherche à se procurer auprès de l'agent de santé.

On constate une diminution de la clientèle des agents DBC qui affirment que leurs clientes étaient plus nombreuses au début de l'intervention. La baisse de la clientèle s'explique par plusieurs facteurs : certaines des clientes initiales ont désiré avoir un enfant et ont interrompu leur traitement, d'autres, en raison de la précarité de la saison et du manque de ressources financières, ont cessé de s'approvisionner, enfin et non des moindres, il y a rupture de stocks de pilules.

$1 \quad$ Quels problèmes sont-ils perçus comme les plus urgents à résoudre ?

- La construction d'un logement pour l'école

- La réalisation d'un forage à côté de l'école. 
- La construction d'un barrage

- La construction d'un dispensaire

2 Quels problèmes de santé sont-ils perçus comme les plus urgents à résoudre ?

- La méningite, la toux, la diarrhée des enfants.

- La construction d'une pharmacie villageoise.

3 La fécondité non maîtrisée constitue-t-elle un problème qui peut être résolu par la communauté $?$

Non.

La fécondité non maîtrisée n'est pas une préoccupation du moment pour les villageois. Ce qui est important à leur yeux c'est de résoudre des problèmes de santé courants : méningite, toux, paludisme.

L'agent DBC est un laafi geta (celui qui s'occupe de la santé) comme l'ancien agent PSP.

Ne pas être malade du paludisme, ne pas avoir la toux, la diarrhée et les autres maladies c'est avoir le laafi (santé).

L’espacement des naissances n'est pas pensé et vécu comme une maladie.

Les villageois que nous avons rencontrés à Bangma (le chef de village (naaba) entouré du chef des jeunes (kam naaba) et de sept autres personnes) ne perçoivent pas la maîtrise de leur fécondité comme un besoin urgent et prioritaire qui doit être résolu par la collectivité. Les besoins urgents et prioritaires sont la toux, la méningite, les diarrhées des enfants, le manque de médicament de première nécessité, d'un logement pour le maître d'école, un forage.

\section{b. Les villageois soutiennent-ils l'intervention ?}

1 Que connaissent les enquêtés des activités de l'agent DBC ?

Les populations n'ont pas une idée précise des activités que mènent l'agent DBC : soit elles l'appellent « docteur » soit «celui qui possède le médicament pour espacer les naissances ». On les reconnaît à leur sacoche et à leur bicyclette. On les confond souvent aux agents de santé communautaire car la plupart exerçaient ces fonctions auparavant.

«Ce sont des gens qui sont dans le village, qui circulent avec un vélo de la FS». 
«Certains portent un gros sac de logtore et vendent de l'aspirine de la nivaquine et le rog yaagr tiim (le médicament de l'espacement des naissances)».

2 Quels sont les avantages matériels et sociaux que les villageois retirent de l'intervention?

A Bangma et Nabélé où nous avons pu réunir des gens pour la discussion dirigée, il est ressorti que l'intervention leur permet d'acheter facilement de l'aspirine et de la nivaquine.

3 Que comprennent-ils du travail des agents DBC?

Certains des enquêtés savent qu'il existent des gens dans le village qui ont été formés et disposent d'un vélo de la FS, d'une sacoche, parfois deux vélos et d'une grosse caisse pour médicaments. Il y a une confusion avec les anciens agents PSP reconduits.

D'autres par contre, comme le chef de Nabélé, disent ne pas connaître le travail des agent DBC. Le chef de Nabélé dit que ce dont il se rappelle, c'est lorsque des gens sont venus lui demander de choisir deux personnes pour s'occuper de la santé. Par la suite ceux qui ont été choisis sont revenus lui montrer un vélo et une sacoche. Depuis lors ceux ci n'ont plus donné de suite. Il ne sait ni ce qu'ils font ni ce qu'ils gagnent.

4 Que comprennent-ils du rôle du comité de gestion dans les activités de DBC ?

A Bangma et Nabélé où la discussion dirigée a eu lieu, les participants ne connaissent pas le rôle du comité de gestion. A Nabélé ils ignorent jusqu'à l'existence du comité de gestion. Un grand nombre de personnes ignorent l'existence d'un représentant du comité de gestion au sein du village.

Les villageois de Bangma reconnaissent et louent les mérites de l'accoucheuse villageoise qui effectue consciencieusement son travail bien qu'elle ne dispose pas de vélo. Elle n'a pas été choisie pour devenir DBC. Ces villageois comparent le travail de l'accoucheuse villageoise à celui des DBC qui ne jouent toujours pas correctement leur rôle. Ils citent ainsi le cas de l'agent DBC homme qui a deux vélos (PSP et DBC) et ne fait pas son travail. 
Les enquêtés ignorent le rôle et la fonction du comité de gestion, parfois même son existence. C'est le cas des enquêtés de Nabélé et Bangma.

Non. Ils ne sont pas motivés pour des activités dont ils se méfient et ont honte de cautionner publiquement la vente de contraceptifs car la norme en vigueur dans le milieu est d'avoir un nombre suffisant d'enfants des deux sexes.

$8 \quad$ Quel est leur niveau de participation?

Le niveau de participation des villageois dans l'intervention s'est limité au choix des représentants pour le comité de gestion et des agents DBC, il y a deux ans. Depuis lors, ils n'ont plus été associés à une quelconque activité concernant la DBC.

En ce qui concerne le suivi et le contrôle du travail des DBC, les enquêtés disent que c'est le domaine du personnel de la FS. Il en est de même de l'entretien de la bicyclette.

\section{Quel est leur niveau d'appropriation?}

Les enquêtés ne se sont pas appropriés l'intervention. Personne n'a évoqué des changements qui seraient intervenus du fait de l'intervention.. Aucun propos ne donne une légitimité à l'intervention. C'est de la vente de l'aspirine et de la nivaquine dont parlent les villageois à propos de l'intervention.

Le soutien des enquêtés de Bangma et Nabélé n'est pas total. Il se situe seulement au niveau du choix des représentants au comité de gestion et des agents DBC.

Pour ce qui est du suivi et du contrôle du travail des DBC, ils s'estiment incompétents et non responsables. Aucun compte n'est demandé aux représentants dans le CG et aux agents DBC à tel point que les villageois refusent même de prendre l'initiative de changer les agents DBC qui s'illustrent par leurs mauvaises prestations. C'est le cas de l'agent DBC homme de Bangma qui ne se rend pas aux invitations du chef.

Selon les enquêtés, il y a un domaine bien délimité pour l'action de tout un chacun :

"Quelqu'un qui ne connaît rien en matière de santé (c'est à dire eux) ne peut pas et ne doit pas se mêler du travail de celui qui s'occupe de la santé (c'est à dire le laafi geta : agent DBC). Sinon on dira qu'il est jaloux du travail de l'autre ou qu'il est jaloux de son vélo et de sa sacoche. » 


\section{c. Les agents DBC sont-ils motivés ?}

\section{$1 \quad$ Les agents DBC croient-ils en l'intervention?}

Les discours tenus par les agents DBC montrent à l'évidence qu'ils comprennent l'intervention mais rien ne nous permet de dire qu'ils y adhèrent puisque beaucoup ne la pratiquent pas dans leur propre famille. Ils considèrent que les populations n'ont pas encore bien compris l'intérêt d'une telle intervention mais des changements dans les mentalités commencent à s'observer. Ils relèvent que les populations écoutent les causeries mais ne changent pas pour autant leur comportement. Certains agents ont été traités de mauvais, ninyooro (méchant) car ils proposent un produit pour espacer les naissances alors que leur propre femme continue à accoucher régulièrement

2 Quels sont les problèmes rencontrés par les agents DBC dans le cadre de leurs activités ?

Les problèmes sont de nature diverse

- problèmes matériels : l'entretien du vélo est difficile et les bénéfices tirés de la vente des produits ne suffisent pas à couvrir les frais occasionnés par l'entretien du vélo.

- problèmes d'approvisionnement des produits (rupture de stock)

- $\quad$ problèmes de la faiblesse des bénéfices qui ne permettent même pas de réparer le vélo. Pour l'aspirine et la nivaquine qui se vendent bien, les agents n'ont aucune idée du bénéfice net qu'ils peuvent en retirer. Les autres produits se vendent à un très faible rythme.

- problèmes de temps disponible : en saison des pluies, il y a très peu de temps pour mener les activités (causeries et visites à domicile). Les villageois eux-mêmes sont occupés par les travaux champêtres. Les accoucheuses villageoises DBC sont parfois sollicitées pour assister des accouchements qui nécessitent un long temps de présence dans le cas des primipares : elles doivent accompagner la parturiente jusqu'à la maternité.

- Problème de distance à parcourir dans certains cas : certains villages sont très étendus et les agents ne peuvent couvrir toutes les concessions (cas de Kalzi où les cinq quartiers sont très distants l'un de l'autre).

- Problème d'accessibilité culturelle : en milieu protestant, la sensibilisation est très difficile à tel point qu'en deux endroits, tant le personnel de la formation sanitaire que l'agent DBC considèrent que c'est inutile de 
prolonger l'expérience car les populations sont hostiles aux contraceptifs, en raison de leurs convictions religieuses.

- Certains produits ne se vendent pas : les agents DBC féminins ont cédé leur stock de capotes aux hommes. La pilule de type «ovret» ne se vend pas car rares sont les femmes qui voient leurs règles revenir avant une année après l'accouchement.

- Les populations, en raison de la famine n'ont plus les moyens d'acheter les contraceptifs : certaines clientes ont même pris la pilule à crédit.

- Les jeunes ont honte de s'approvisionner auprès d'eux, qui sont de la même génération que leurs parents. Il est d'ailleurs plus facile de s'approvisionner discrètement sur le marché, au même prix.

3 Quelles sont les autres activités et responsabilités des agents DBC ? disponibilité pour les activités DBC

Les agents DBC ont tous d'autres activités et responsabilités. Ceux qui sont le plus actifs pour la stratégie DBC sont aussi engagés dans différentes responsabilités: groupement villageois, périmètre maraîcher, associations à caractère social. En saison des pluies ils sont très occupés et de ce fait peu disponibles pour les activités si ce n'est la vente des produits à domicile, le soir.

Ceux qui ont d'autres activités rémunératrices, les jours de marché par exemple, sont encore moins disponibles. Les femmes DBC qui sont accoucheuses villageoises ont une surcharge de travail. D'un côté les accouchements constituent une occasion de sensibilisation pour éviter des grossesses rapprochées, d'un autre côté le temps consacré aux accouchements est un handicap pour mener d'autres activités comme les causeries ou visites à domicile.

\section{$4 \quad$ Combien de temps les agents DBC consacrent-ils à l'intervention ?}

Cela dépend de la saison. Durant la saison des pluies aucun agent DBC n'a tenu une causerie car les populations étaient occupées par les travaux champêtres. En général, c'est dans la soirée que les gens viennent les voir pour se procurer les produits. Un agent DBC quant à lui emporte sa sacoche sur son périmètre de maraîchage et peut ainsi servir les éventuels clients. 
Que comprennent les agents DBC de leurs tâches et de leur rôle?

Certains se sentent utiles en mettant à la disposition des populations des médicaments de première nécessité. Les anciens agents PSP ont le sentiment qu'ils continuent le même travail (agents de santé communautaire). Ils comprennent leur rôle et leurs tâches dans l'intervention.

$6 \quad$ Jouent-ils leur rôle dans l'intervention?

Compte tenu des différents problèmes évoqués précédemment, les agents DBC ne peuvent jouer efficacement leur rôle car leur motivation s'est érodée au fil des temps.

$7 \quad$ Les agents DBC sont-ils satisfaits de la supervision?

A la question de savoir si le superviseur vient leur rendre visite, les agents répondent par l'affirmative mais lorsque l'on demande des précisions sur la date de la dernière visite, on se rend compte que certains villages n’ont pas été visités récemment.

En effet, la supervision est irrégulière et c'est à l'agent de se déplacer jusqu'à la formation sanitaire pour déposer son rapport mensuel. Certains agents s n'ont pas reçu la visite du superviseur depuis plus de neuf mois.

\section{$8 \quad$ Les agents DBC font-ils confiance à leur superviseur ?}

Ils ne le voient pas régulièrement mais ne s'en plaignent pas ouvertement.

9 Quelle est la fréquence des rapports et des réunions avec laquelle les agents DBC rencontrent leurs superviseurs pour leur faire le point ? (ordre du jour et contenu des réunions)

Les trois formations sanitaires visitées ont des modalités de fonctionnement différentes :

A Vipalogo, en principe le comité de gestion se réunit avec les agents DBC une fois par mois ainsi que pour des activités ponctuelles comme l'organisation d'un laafi raaga. Cependant, de façon pratique, il ne s'est pas réuni lors de la dernière saison des pluies.

A Komki, il semble qu'il y ait une moins grande régularité et plus de problèmes : le précédent comité de gestion a été renouvelé et lors de la réunion, les membres présents le jour de leur réunion ordinaire étaient moins nombreux. Les agents DBC ne participent pas aux réunions sauf ceux qui cumulent des fonctions de représentant du village et de DBC. Néanmoins l'accoucheuse auxiliaire fait des sorties régulières pour 
relever les rapports. Notons que la distance qu'elle a à parcourir est moins grande ainsi que le nombre de villages à superviser.

A Komsilga, en principe, les agents vont à la formation sanitaire tous les 25 du mois remettre leurs rapports, mais ils sont de plus en plus irréguliers et il y a beaucoup d'absents.

10 Quelle est la fréquence avec laquelle les agents DBC parlent à leur comité de gestion de leurs activités?

Selon eux, c'est à la formation sanitaire qu'ils rendent des compte en leur remettant leurs rapports. Certains agents ont également des contacts avec le délégué administratif de leur village mais pour la plupart ce ne sont que des contacts ponctuels pour donner des informations précises (tenue d'un laafi raaga par exemple). Un chef s'est plaint de ne plus être informé des activités en depuis la remise de matériel. Selon les comités de gestion, cela ne fait pas partie de leur mandat.

11 Les agents DBC savent-ils à qui ils doivent rendre des comptes ?

Oui.

12 A qui les agents DBC rendent-ils effectivement compte?

Ils rendent des comptes au personnel des formations sanitaires et plus spécifiquement à la personne responsable de la PF

13 La ristourne sur les produits vendus constitue-t'elle une motivation?

La ristourne qui est très modeste puisque la vente des contraceptifs est très réduite ne suffit même pas dans la plupart des cas à réparer la bicyclette. Le bénéfice sur la vente de l'aspirine et de la nivaquine n'est pas encore connu alors que cela se vend bien et pourrait constituer une sorte de compensation au manque à gagner sur la vente des contraceptifs.

14 Quel est le degré de remplissage des instruments de gestion ? (qualité)

C'est vraiment là l'un des points le plus faible :

- $\quad$ les fiches ne sont pas comprises par les agents 
- le remplissage en est difficile : même les agents lettrés ont du mal à remplir leurs fiches car ils oublient de reporter la date (mois) sur chaque fiche et n'ont pas compris le mode de remplissage.

- Enfin, les fiches sont en nombre insuffisant à tel point qu'il faut inscrire les données de plusieurs mois sur la même fiche alors que celle-ci est mensuelle.

\section{d. Les comités de gestion sont-ils efficaces ?}

$1 \quad$ Les membres du comité de gestion croient-ils en l'intervention ?

Lorsque les membres du comité de gestion parlent des DBC, ils évoquent la facilité d'accès à l'aspirine et à la nivaquine. Il ne ressort pas toujours une différence perceptible entre travail de DBC et travail des anciens agents PSP.

Ils croient que l'intervention permettra effectivement aux villageois d'avoir accès à l'aspirine et à la nivaquine qui sont des médicaments très sollicités. Il est dès lors difficile de soutenir que les membres des CG croient à l'intervention si elle ne se résume pas à vendre seulement de l'aspirine et de la nivaquine.

Les CG ne comprennent donc pas l'intervention, ils ne peuvent de ce fait pas la soutenir et la faire progresser.

2 Quels sont les problèmes rencontrés par les membres du comité de gestion ?

Les problèmes sont d'ordre matériel et personnel. Les membres du CG se plaignent de ne pas recevoir un salaire, de ne disposer d'un moyen de locomotion.

Certains demandent des sacoches pour leurs papiers.

Pour certains membres du CG, c'est perdre du temps que de participer au activités du CG. Ils auraient mieux gagner leur temps en se consacrant à leurs activités telles que les récoltes, le maraîchage, le petit commerce.

3 Quelles sont les autres activités et responsabilités des membres de comités de gestion?

Les autre activités des membres du CG sont multiples. Il y a :

- l'activité saisonnière principale qu'est l'agriculture

- les activités de contre-saison telles que le maraîchage, le petit commerce

- les activités associatives dans les groupement villageois, les groupes d'animation culturelle villageoises (chants et danses), les groupes d'animation religieuse, les association de développement local. 
L'implication des membres des CG dans d'autres activités et responsabilités ne leur permet pas de disposer d'assez de temps pour s'occuper de la DBC.

Exemple de membre de comité impliqué dans d'autres activités et ayant des responsabilités Responsabilité dans le comité de gestion de Komsilga secrétaire Autres responsabilités

1- $\quad$ secrétaire du projet RECIF Komsilga

2- $\quad$ secrétaire du groupement Waog taaba de son quartier

3- Secrétaire adjointe de l'association des parents d'élèves (APE) Komsilga

4- trésorière de l'association des volontaires français du progrès (AVFP) Komsilga

5- Enseignante en langue moore (karêmoor karêsaamba) de Komsilga

6- vendeuse de légumes à Komsilga et à Kienfangué

4 Combien de temps les membres du comité de gestion consacrent-ils à l'intervention DBC ?

Les CG ne consacrent que très peu de temps à l'intervention.

Ce sont plutôt les agents DBC qui semblent consacrer beaucoup de temps aux activités des CG en y occupant des postes de responsabilité.

Par exemple, nous avons constater la présence massive d'agents DBC à deux réunions du CG de Vipalogo. En effet, à la réunion de comité du 27 octobre 98, 12 agents DBC y ont pris part sur un total de 22 personnes présentent. Ce fut un agent DBC qui assura le secrétariat.

A celle du 30 octobre 98, les agents DBC étaient au nombre de 13 sur un total de 17 présents. 
Sur trois comité des gestion rencontrés, un seul à consacré une seule réunion pour discuter du bon usage des vélos DBC. La dite réunion s'est tenue sur initiative du responsable de la formation sanitaire.

Les deux autres comités n'ont consacré aucune réunion sur la DBC

$6 \quad$ Le comité de gestion prend-il des décisions relatives à la DBC ?

Non.

Aucune décision n'a été prise relative à la DBC au niveau des CG rencontrés.

Il y a eu une simple mise au point sur le bon usage des vélos.

$7 \quad$ Le comité de gestion rend-il des comptes aux villageois ?

Deux points de vus opposés apparaissent : celui du comité et celui des villageois.

Le comité de gestion dit qu'il rend compte régulièrement par le truchement de ses représentants au village. En effet, chaque village est représenté dans le CG par deux personnes.

Mais certains représentants ne sont pas toujours présents aux réunions des CG. Par exemple à la réunion du CG de Komsilga le 1er décembre 98, 11 personnes étaient présentes sur un total de 30 personnes.

Les représentants des différents villages disent également qu'ils rendent compte aux villageois après chaque rencontre.

Pour les villageois, le CG ne leur rend pas de compte.

En effet, il à été dit lors d'une discussion dirigée de groupe tenue à Bangma village de Komsilga (avant la réunion du CG de Komsilga), que leur représentant au comité ne leur rend compte de rien. Interrogé lors de la réunion du comité, il affirma le contraire. Ce dernier soutenait que l'agent DBC homme du village fait correctement son travail alors que le chef et les participants à la discussion soutiennent le contraire. Ils souhaitent que l'agent DBC soit remplacé car il refuse même de répondre à leur appel.

On pourrait se demander si le représentant y va pour recevoir de l'argent ou pour l'intérêt du village ? Dans deux comités, ils reçoivent de l'argent quand ils assistent aux réunions. En effet, à Komsilga, les membres 
présents à chaque réunion du comité se partagent 5000F CFA. C'est la forme de motivation que le comité a décidé de mettre en place.

A Vipalogo, le CG ne rencontre les délégués administratifs de village que lorsqu'il y a une grande tâche à accomplir. Depuis un an, ils ont été conviés par le comité une seule fois pour prendre part aux travaux d'implantation d'une maison au sein de la FS. Les délégués sont libres de prendre part à toute autre activité du CG.

8 Quelles sont les dernières décisions prises et mises en œuvre relatives aux activités DBC ?

En dehors d'une mise au point sur l'utilisation des vélos à Vipalogo, il n'y a pas eu d'autres décisions prises et mises en œuvre relatives aux activités DBC au niveau des CG rencontrés.

$9 \quad$ L'activité est-elle intégrée au plan d'action du comité de gestion?

Non.

Les plans d'action des CG s'articulent autour d'activités pouvant rapporter de l'argent :

- laafi raaga : il s'agit d'un marché organisé ponctuellement au cours duquel le CG en collaboration avec le personnel de la FS, organisent un ensemble d'activités de prestation de service de santé

- plantation d'arbres (eucalyptus) dans le but d'obtenir un peu d'ombre et surtout de revendre le bois

- quête d'argent dans chaque quartier

- quête de céréales

\section{e. Le personnel des formations sanitaires est-il motivé par la participation des agents DBC et des comités de gestion?}

$1 \quad$ Le personnel des FS croit-il en l'intervention ?

Certains de ceux qui n'ont pas été formés donnent l'impression de se sentir étrangers à la stratégie : ils ne l'ont pas appropriée et considèrent que cela ne vaut pas la peine de continuer. Par contre d'autres semblent l'avoir approprié et s'impliquent dans le travail.

Un responsable d'une formation nous a avoué qu'il est revenu sur son impression de départ et que maintenant il croit que l'on peut augmenter l'utilisation de la contraception avec les agents DBC. Il doutait 
de la capacité des DBC à effectuer leur mandat. En effet, selon lui, les critères de choix des agents par la communauté n'étaient pas assez rigoureux, notamment la sélection n'exigeait pas la connaissance de l'écriture.

Certains y croient, d'autres non.

$2 \quad$ Le personnel des FS croit-il que l'on peut augmenter l'utilisation de la contraception ?

Pour l'ensemble du personnel, c'est une opération à très long terme car cela concerne le changement des mentalités et des comportements.

3 Le personnel des FS croit-il que la participation des agents DBC peut augmenter l'utilisation de la contraception?

Un seul membre du personnel des FS a indiqué sans ambiguïté que la présence des agents DBC dans les villages augmente la sensibilisation des populations aux méthodes contraceptives ainsi que la clientèle. Néanmoins, il relève que ce sont seulement les femmes qui veulent des contraceptifs agissant à plus long terme comme les injections qui se présentent à la formation sanitaire, référées par les agents DBC.

4 Le responsable de la PF de la FS croit-il que la formule DBC devrait être étendue à d'autres zones ?

A l'exception d'un responsable qui est favorable à l'extension sous réserve que les agents soient alphabétisés, les autres considèrent que c'est prématuré.

Un considère qu'il vaut mieux arrêter l'expérience, un autre a d'abord déclaré cela mais est revenu sur sa déclaration pour dire que c'est une bonne stratégie. Ceux qui n'ont pas été formés peuvent manquer de motivation et d'intérêt pour une opération pour laquelle ils n'ont pas été préparés.

5 Le personnel de santé a-t-il déjà entendu parler de problèmes dans son aire de travail concernant les agents DBC ou le comité de gestion?

Il nous a été relaté le cas d'un agent DBC qui ne faisait pas consciencieusement son travail et sur plainte du délégué administratif de son village, le matériel a été retiré par le comité de gestion et une autre personne a été responsabilisée.

Un comité de gestion a entendu parlé des problèmes relatifs à l'utilisation des vélos. Il a convoqué les agents pour attirer leur attention sur le soin à accorder à ces outils de travail. 
Trois agents DBC ont abandonnés leur travail et leur village pour aller en Côte d'Ivoire, un autre est allé s'installer à Ouagadougou.

6 Le personnel de santé a-t-il déjà entendu parler de problèmes dans son aire de travail concernant les comités de gestion?

Le comité de gestion d'une formation sanitaire a beaucoup de difficultés à se réunir en raison de problèmes survenus par le passé. Le bureau a d'ailleurs été renouvelé. Il semblerait qu'il y ait eu des problèmes de gestion des fonds.

$7 \quad$ Les superviseurs ont-ils reçu la formation spécifique ?

Sur les trois formations sanitaires visitées, seules le personnel d'une formation a été formé initialement. Les autres personnes ont été affectées dans les formations une année après le lancement de la stratégie et aucune formation n'a été dispensée à leur intention.

$8 \quad$ Les superviseurs ont-ils été mutés depuis le début de l'intervention?

Il n'y a qu'une formation sanitaire qui a conservé le personnel en poste au moment du lancement de l'expérimentation.

9 Quel est le niveau de soutien, d'engagement du personnel auprès des agents DBC ?

En dehors du personnel formé d'une formation sanitaire et d'une autre personne, il ne semble pas qu'il y ait un réel engagement du personnel auprès des agents DBC pour diverses raisons.. 


\section{f. Les responsables de district sont-ils motivés par l'approche communautaire ?}

1 Les responsables des districts croient-ils que l'on peut augmenter l'utilisation de la contraception?

2 Les responsables des districts croient-ils que la participation des agents DBC peut augmenter l'utilisation de la contraception?

3 Les responsables des districts croient-ils que la formule DBC devrait être étendue à d'autres districts ?

4 Les responsables des districts ont-ils déjà entendu parler de problèmes concernant les agents $\mathrm{DBC} ?$

5 Les responsables des districts ont-ils déjà entendu parler de problèmes concernant les comités de gestion?

Les conflits entre le personnel de santé, les agents DBC et les membres comités de gestion sont inévitables. Les responsables des districts sont-ils conscients de ces problèmes ? Comment les résolvent-ils ?

$6 \quad$ Les responsables de districts ont-ils reçu la formation spécifique ?

$7 \quad$ Les responsables de districts ont-ils été mutés depuis le début de l'intervention?

8 Quel est le niveau de soutien, d'engagement des responsables de district auprès des agents $\mathrm{DBC}$ ?

$9 \quad$ L'activité DBC est-elle prévue dans le plan d'action du district?

Le responsable de district vient d'être affecté et son remplaçant par intérim nous a demandé de l'informer de la stratégie. 
Tableau 28 : Stratégie DBC - points forts et points faibles

\begin{tabular}{|c|c|c|}
\hline Niveau & Points forts & Points faibles \\
\hline Agents DBC & $\begin{array}{l}\text { Légitimité } \\
\text { Possession de matériel spécifique } \\
\text { Proximité et accessibilité physique } \\
\text { Motivation individuelle }\end{array}$ & $\begin{array}{l}\text { Motivation matérielle insuffisante : découragement } \\
\text { Formation insuffisante } \\
\text { Peu de lettrés } \\
\text { Grande charge de travail }\end{array}$ \\
\hline Formations sanitaires & $\begin{array}{l}\text { Certains sont motivés } \\
\text { Certains s'impliquent même sans } \\
\text { formation }\end{array}$ & $\begin{array}{l}\text { Mobilité du personnel } \\
\text { Supervision des DBC irrégulière } \\
\text { Surcharge de travail } \\
\text { Pas de recyclage } \\
\text { Sentiment d'abandon par leurs responsables }\end{array}$ \\
\hline Logistique & Moto et carburant & $\begin{array}{l}\text { Remplissage des fiches compliqué } \\
\text { Problèmes d'approvisionnement : rupture de stocks, } \\
\text { produits périmés non remplacés }\end{array}$ \\
\hline Comités de gestion & & Pas de lien avec la stratégie DBC \\
\hline Villages & La santé est prioritaire & $\begin{array}{l}\text { Contraception non prioritaire } \\
\text { Mobilisation difficile } \\
\text { Pas de contrôle effectif de l'agent DBC }\end{array}$ \\
\hline
\end{tabular}

Tableau 29 : Stratégie DBC - problèmes rencontrés.

\begin{tabular}{|l|l|l|}
\hline Agents DBC & Comité de gestion & Personnel FS \\
\hline & Problèmes matériels & \\
& Moyen de déplacement & \\
& Sacoche & \\
& Rémunération & \\
\hline & Problèmes individuels & \\
& Perte de temps non compensée & \\
\hline
\end{tabular}

\section{Alternative DBC formation sanitaire}

Les avis sont partagés :

- Les agents DBC sont utiles pour la sensibilisation mais par souci de discrétion certains clients vont s'approvisionner à la formation sanitaire. 
- Le personnel des formations affirme qu'il y a accroissement de la consommation de produits contraceptifs suite au travail des DBC (à vérifier).

- En d'autres termes, on peut dire qu'il y a complémentarité entre les deux, ce qui mériterait une meilleure concertation et un travail en partenariat.

- Un des aspects qui peut constituer un obstacle c'est que lorsqu'une femme veut des produits contraceptifs, on lui demande si son conjoint est d'accord. pour ne pas avoir de problèmes avec lui. Bien sûr, dans certains cas, par exemple celui d'une femme qui a déjà six ou sept enfants et qui est fatiguée, on y fait dérogation. La plupart des jeunes filles ne peut donc s'approvisionner auprès des DBC et le fait sur la place du marché :

- « c'est les copains des filles qui viennent pour chercher de l'aide car les filles se disent que je suis comme leur mère et comme elles ne sont pas mariées, elles ont peur de venir ». 


\section{RECOMMANDATIONS}

\subsubsection{Principe DBC :}

Si on admet que la planification familiale n'est pas un besoin prioritaire à l'heure actuelle pour la majorité des populations, pour preuve l'abandon des clientes par manque de ressources pendant la période de soudure particulièrement pénible cette année, on peut en conclure que la pertinence est douteuse quant à la planification familiale.

L'extension de la stratégie mérite d'être repensée dans sa forme et dans son fonds. Une réflexion approfondie doit être menée avant d'entreprendre l'extension.

Formation et évaluation continue des agents DBC et du personnel des formations sanitaires.

Multiplier le nombre de DBC dans les villages où il y a plusieurs quartiers éloignés les uns des autres, pour assurer un encadrement plus efficace.

Nous n'avons pas perçu la notion de bien collectif et d'utilité publique en ce qui concerne la stratégie DBC. Les différents intervenants travaillent pour eux-mêmes, non pour la collectivité. Les comités de gestion sont occupés à gérer les fonds obtenus par la vente des médicaments, les agents DBC sont préoccupés des ristournes des produits vendus et l'idée communautaire est absente des discours comme des comportements. La stratégie communautaire ne peut donc pas être durable dans un tel contexte.

\subsubsection{Logistique, encadrement}

Approvisionner régulièrement les formations sanitaires en produits non périmés

Le maillon le plus faible est l'approvisionnement en produits : par exemple, la formation sanitaire de Vipalogo doit se rendre obligatoirement à Sapone alors que les voies de communication sont difficiles. Certains

- Il faut s'assurer de la régularité de la supervision: il faut de trois a cinq jours pour pouvoir encadrer tous les villages et c'est un surcroît de travail non compensé.

- Concevoir des fiches de suivi plus simples et alphabétiser les agents pour qu'ils puissent les remplir correctement

- Assurer une formation et évaluation permanente

- Etudier les besoins spécifiques des jeunes et la manière de les satisfaire 
En l'absence de participation communautaire et d'incitation financière, seul le Ministère peut motiver les agents DBC. Il doit pour cela assurer une présence quasi permanente sur le terrain, organiser régulièrement des formations, évaluer de façon mensuelle le travail des DBC et recruter des agents DBC de façon régulière (on est motivé et on travaille mieux la première année). Il doit montrer de la rigueur dans sa propre gestion : pas de rupture de stock, pas d'absentéisme, etc. La faiblesse des prestations étatique ne doit pas servir de justification a l'inactivité des agents DBC. Le ministère en a t il la capacité ?

\subsubsection{Au niveau communautaire}

Recruter plus de DBC, autrement.

- Pour plus d'engagement des agents, instituer un contrat de durée limitée renouvelable si la population est satisfaite de leurs prestations.

- Sélectionner les agents DBC en fonction de leur potentiels et non selon leur relation avec les membres de la «nomenklatura » villageoise.

- Campagne de recrutement des DBC doivent servir à promouvoir la santé communautaire, et donner du prestige à la fonction d'agent DBC.

- Recruter un plus grand nombre de DBC et les faire tourner plus rapidement (missions d'un an, renouvelables pour les agents les plus efficaces) pour éviter qu'elles ne se fonctionnarisent. Les mettre en concurrence. Il est plus facile de motiver agents pendant 1 an que sur une période longue et indéterminée. Les agents les plus performants deviennent les formateurs et/ou superviseurs des nouvelles recrues, offrant ainsi des perspectives de «promotion » aux agents.

- Ainsi, au bout de 5 ans, plus de 400 hommes et femmes auront été formés et auront promu la PF dans la zone $\mathrm{B}$, soit en moyenne 10 personnes par village, contre deux ou trois avec le système actuel. La masse critique de personnes «compétantes » en PF dans chaque village sera plus rapidement atteinte. Les succés de la stratégie DBC dans chaque village dépendra moins du bon vouloir d'un ou de deux agents DBC.

Mener de la sensibilisation pendant la consultation du nourrisson : nous avons l'exemple d'une formation sanitaire où l'agent DBC avait l'habitude de faire de la sensibilisation les jours de consultation SMI : en effet les femmes sont nombreuses à assister à cette consultation d'autant plus qu'il y a distribution de vivres.

Activités et service de PF spécifique aux adolescents 
Education sexuelle pour que les femmes maîtrise mieux leur fécondité de façon naturelle. Les méthodes naturelles et traditionnelles représentent un premier pas vers l'adoption de contraceptifs modernes. 


\section{ANNEXES}

\section{Annexe A Explications complémentaires sur le schéma d'évaluation}

(Voir la Figure 1 page 30)

Afin d'analyser les processus par lesquels l'intervention peut avoir un impact, nous distinguons les déterminants opérationnels des déterminants contextuels. Les déterminants opérationnels (qui sont encadrés dans la Figure 1) sont visés directement (flèches épaisses) ou indirectement (flèches fines) par l'intervention. Par exemple, la promotion et la distribution de contraceptifs constituent des outputs du programme visant à modifier les perceptions et l'utilisation de contraceptifs. En revanche, on ne s'attend pas à ce que le programme ait un effet sur les déterminants contextuels. Par exemple, sur les caractéristiques individuelles ou communautaires, ou encore, à moyen terme, sur la mortalité infantile. Les déterminants contextuels et opérationnels sont reliés dans un processus en chaîne (flèches) conduisant à un résultat final (la réduction de la fécondité).

L'intervention s'insère dans un paradigme économique dans lequel les individus décident de limiter ou non leur fécondité sur la base d'une analyse coûts-bénéfices. Cela ne signifie pas pour autant que la culture soit absente de ce processus. Bien au contraire, dans le contexte du Bazèga d'aujourd'hui, les bénéfices économiques d'une fécondité limitée apparaissent être largement étouffés par son coût social. L'intervention ne réduira pas le coût social d'une fécondité limitée : ce n'est pas dans ses objectifs (d'où l'absence de flèches entre l'intervention et l'intention de limiter la fécondité dans la Figure 1. Au mieux, des considérations économiques ou de santé au niveau familial peuvent compenser la pression sociale pro-nataliste que subit l'individu. Les individus entreprennent aussi une analyse coûts-bénéfices en décidant d'utiliser une méthode contraceptive plutôt qu'une autre ou en préférant la contraception à l'avortement. Convaincre des femmes de limiter leur fécondité n'entraîne pas obligatoirement une utilisation accrue de contraceptifs mais peut, à la place, accroître la prévalence de pratiques traditionnelles telles que l'abstinence post-partum et l'avortement clandestin (6).

Les hypothèses de l'intervention peuvent être analysées en termes d'offre et de demande. Du côté de la demande, l'intervention suppose qu'il existe aujourd'hui une demande insatisfaite de méthodes modernes de planification familiale. En effet, selon les chiffres de l'EDS, respectivement $20 \%$ et $9 \%$ des femmes mariées enceintes auraient préféré attendre avant leur prochaine grossesse ou même de ne pas avoir un enfant 
[DHS, 1993]. Il est probable que ces chiffres soient plus faibles en milieu rural. La survie de l'enfant apparaît du côté de la demande puisque la plupart des démographes s'accordent pour dire que la réduction de la mortalité infantile et juvénile réduit la demande d'enfants. La prise en compte d'une baisse de la mortalité dans les décisions de fécondité ne sont pas immédiates mais prennent au contraire prendre du temps. C'est pourquoi nous ne nous attendons pas à observer, en moins de 5 années d'intervention, l'effet d'une amélioration de la survie de l'enfant sur la fécondité (flèches 5 vers 1). C'est pour cela que nous considérons la survie de l'enfant comme un déterminant contextuelle - qui constitue un obstacle aux efforts de la DBC plutôt qu'un déterminant opérationnel.

Du côté de l'offre, les objectifs de l'intervention sont fondés sur l'assertion que les services existants de PF ne sont pas pleinement utilisés car leur qualité est inadéquate et les formations sanitaires coupées des villages. C'est ici que l'intervention est supposée avoir un effet direct et fort. En augmentant la qualité des services de PF et l'accessibilité aux contraceptifs modernes, on espère accroître la proportion de femmes utilisant les contraceptifs (7) et qui limitent avec succès leur fécondité. Avec la promotion de la PF et des méthodes contraceptives modernes, on espère que les perceptions de l'utilisation de la contraception changent (9) et qu'augmente la demande de contraceptifs modernes (8). Dans les zones où les volontaires DBC distribuent des contraceptifs, les perceptions des villageois sur leur utilisation affecteront leur accessibilité (flèche C). C'est une hypothèse classique des interventions communautaires: s'ils pcrçoivent l'utilisation de la contraception comme étant positive, les villageois soutiendront le programme, amélioreront ainsi son accessibilité, son efficacité et sa pérennité.

En résumé, l'intervention postule qu'en améliorant la qualité des services (composante FS) et en mobilisant les villageois (composante communautaire), la demande pour les contraceptifs sera satisfaite, l'utilisation des contraceptifs augmentera et, enfin, la fécondité diminuera. 


\section{Annexe B Analyse de la participation communautaire : cadre théorique.}

\section{Participation communautaire : conditions d'efficacité, de durabilité et de généralisation}

\section{Historique}

Les sources du concept de participation communautaire peuvent être recherchées dans trois précédents historiques $^{12}$ :

- La participation communautaire serait le produit d'une idéologie que l'on dit occidentale, qui défend des valeurs démocratiques, populistes, anarchistes et prône la destruction de l'autorité agissant comme source d'oppression (l'Etat par exemple).

- La participation communautaire serait également le produit d'une idéologie occidentale que l'on dit se réclamer du marxisme et qui se donne pour objectif d'élever la conscience politique des populations. Cette source se rapproche de la première dans le fond, mais peut-être pas dans la forme. Toutefois, l'objectif poursuivi est d'étendre les parcelles de pouvoir à ceux qui se trouvent aux bas de l'échelle.

- La participation communautaire serait enfin le produit de la volonté des pays décolonisés de créer un développement social endogène. Les missionnaires, ayant saisi la portée, l'adapteront à leur action. A partir des années 1970, ce sont les Nations Unies qui l'adoptent dans les programmes de développement social et économique de ses institutions spécialisées.

\section{Où en est-on?}

Les tenants de la participation communautaire font à l'heure actuelle apparaître deux courants non exclusifs :

- l'un affirme que la participation communautaire constitue un moyen pour mobiliser des ressources en vue de contribuer à la réussite des projets de développement, en recherchant l'adhésion de la population ; cette vision a des connotations utilitaristes ;

- l'autre considère que la participation communautaire est un moyen pour véhiculer des changements dans les comportements.

Encore faut-il s'entendre sur la définition de participation communautaire qui selon la déclaration d'Alma Ata est :

\footnotetext{
${ }^{12}$ Fournier, P. L. Potvin et M. Goudreau, 1992, la mesure de la participation communautaire dans les programmes de santé, document préparé pour la conférence annuelle des études africaines, Montréal.
} 
« un processus par lequel les individus et les familles d'une part prennent en charge leur propre santé et leur propre bien-être comme ceux de la communauté, d'autre part développent des capacités de concourir à leur propre développement comme à celui de la communauté » (UNICEF-OMS 1978).

Plusieurs cadres conceptuels ont été élaborés, et de ces cadres passés en revue par Fournier et al (1992) nous retiendrons qu'il est indispensable de préciser le contexte et le mode d'organisation politique, les valeurs qui sous-tendent la participation communautaire pour identifier ses enjeux et les logiques poursuivies par ses promoteurs. En effet, pour les uns il peut s'agir d'introduire des transformations sociales par un renforcement du pouvoir ou une conscientisation, alors que pour d'autres il s'agit de pourvoir aux groupes les plus défavorisés une représentation démocratique et active afin de minimiser les conflits sociaux. Dans le cas de la participation de type utilitariste, les intervenants sont préoccupés par l'efficacité et l'impact de leurs interventions.

On arrive ainsi à trois types de participation :

1. la participation qui vise l'investissement du pouvoir se soucie des groupes les plus dépourvus, cherche à les sensibiliser à leur condition, à les responsabiliser et à leur permettre de développer la capacité de modifier leur situation. Un certain degré de conscience politique doit être atteint avant que les individus se sentent concernés et habilités à amener des changements.

2. La participation démocratique recherche la représentativité des différents groupes, la liberté d'expression, des élections et l'égalité.

3. La participation utilitariste est impulsée de l'extérieur et la finalité définie de l'extérieur. Dans ce cas, la communauté se soucie principalement de répondre aux exigences de l'agence d'exécution.

\section{Comment analyser la participation communautaire ?}

Afin de mesurer la participation communautaire dans un programme de santé, Rifkin \& al (1990) ${ }^{13}$ ont identifié cinq constituants que nous adaptons ici à l'évaluation de l'intervention DBC :

1. identification des besoins : détermination par la communauté des besoins prioritaires de la communauté.

Les villageois ont-ils déterminer quelles étaient leurs besoins prioritaires ? Ont-ils choisit spontanément les objectifs du programmes DBC?

\footnotetext{
${ }^{13}$ RIFKIN S.B. (1990), Participation communautaire aux programmes de santé maternelle et infantile/planification familiale, OMS, Genève
} 
2. leadership : les dirigeants de la communauté comme ceux du programme ont la capacité d'exercer une influence sans recourir à la contrainte.

Les décisions des comités de gestions sont elles prises de façon démocratiques?

3. organisation : les structures, méthodes et mode de fonctionnement sont au main des représentant de la communauté.

Les comités de gestions ont ils pouvoir de décision en matiere de gestion du programme et exercentils ce pouvoir dans l'intérêt de la communauté ?

4. mobilisation des ressources : les actions entreprises pour stimuler la contribution de la communauté au programme sont efficaces et rentables ; les ressources communautaires représentent une proportion significative des ressources du projet.

Quelle est la proportion des ressources communautaire (y compris en nature, tel que le temps des agents DBC et membres des CG, et le système de recouvrement) par rapport au coût total des opérations ? Le système de recouvrement des frais est-il rentable ?

5. gestion : les membres de la communauté ou leurs représentant participent à l'ensemble des activités mises en place pour faciliter la réalisation des objectifs.

A ces cinq critères se superpose un sixième : la légitimite des acteurs, des messages et des objectifs de l'intervention

En effet, l'efficacité d'un programme communautaire dépend de la légitimité des représentant et agents communautaires, (les membres du comité de gestion et les agents DBC) et de leur message, la force de l'une compensant la faiblesse de l'autre. Un message légitime est un message qui traduit les intérêts des populations, leurs besoins, leurs priorités et leurs valeurs.

En s'inspirant de WEBER (1950) ${ }^{14}$ nous pouvons distinguer trois sortes de légitimité : la légitimité rationnelle, la légitimité traditionnelle et la légitimité charismatique.

- La légitimité de type rationnel ou démocratique (fondée sur le droit, la pratique institutionnelle,.sur la science..). C'est celle des acteurs extérieurs, jouant le rôle d'élite, qui veulent encourager la compétence, la rationalité et la légalité. Leur ambition est de développer une culture démocratique en pratiquant notamment des élections. Par exemple les membres des comités de gestion sont élus par les villageois mais ils leurs rendent compte et pourraient être démis de leurs fonctions, selon des textes préétablis. 
- La légitimité de type traditionnelle. C'est celle dont la coutume investit les notables villageois. Les agents de développement - armés de pragmatisme et de réalisme - associent ces notables au programme communautaire. ils s'appuient sur les formes d'organisations et d'entraides endogènes pour agir. Ce qui donne parfois du souffle et de la légitimité à des pratiques coutumières et ancestrales, mais renforce le pouvoir des leaders locaux. Du même coup ils les institutionnalisent et leurs confèrent une légitimité démocratique.

- La légitimité de type charismatique. C'est celle qui repose sur les qualités personnelles du chef : sa capacité innée à se faire respecter, à créer le consensus autour de lui, à mobiliser les gens. Le succès de la mission des agents $\mathrm{DBC}$ et du représentant du comité de gestion repose essentiellement sur leur charisme. En effet, le message de la planification familiale peut apparaître comme peu légitime aux yeux de la population. C'est donc les qualités personnelles et exceptionnelles de l'agent DBC et du représentant dans le comité de gestion qui va leur permettre de faire passer un message, de proposer des soins de type moderne, de communiquer, de convaincre, et d'influer sur les comportements.

Si en définitive le succès de la stratégie DBC doit reposer essentiellement sur le charisme des leaders locaux, des membres du comité de gestion et des agents DBC, l'expérience pourra-t-elle être 1) durable ;2) généralisable.

\section{Conclusion}

Pour mesurer le niveau de participation communautaire l'évaluation de la stratégie DBC s'attache à :

- déterminer les besoins perçus comme prioritaires par la communauté

- caractériser le type de leadership exercé par l'agent DBC et le représentant dans le comité de gestion en analysant leur légitimité

- caractériser les pratiques au sein des comités de gestion.

- analyser les pratiques et les effets de la tarification des contraceptifs, la contribution des villageois

- caractériser la pratique des agents DBC.

14 WEBER, M. 1950, le savant et le politique, Paris, Plon 


\section{Annexe C Aspects éthiques de l'évaluation.}

\section{Extrait du protocole d'évaluation :}

Avant l'entretien, les personnes enquêtées sont informées des objectifs de l'enquête d'évaluation. Ils ne sont pas contraints ou payés pour participer à l'enquête de suivi, aux entretiens dirigés de groupes ou aux entretiens individuels semi-directifs. Aucune pression n'est exercée sur eux pour répondre à une question particulière. On leur rappelle qu'ils peuvent cesser d'être interrogés à n'importe quel moment, selon leur convenance. Leurs réponses sont strictement confidentielles. Leur identité n'est pas consignée dans la base de données. Les questionnaires sont stockés à l'UERD à Ouagadougou.

Il est interdit aux enquêteurs et aux superviseurs de suggérer des réponses ou de dispenser des conseils en matière de santé ou d'autres sujets d'ordre privé. Ils ne sont pas autorisés à donner des cadeaux et autres dons aux enquêtés ou à leur famille. Ils doivent respecter la dignité des enquêtés, leur droit à l'intimité et leur liberté de penser.

Si l'enquête révèle qu'un des membres des ménages est sévèrement malade, la famille sera incitée à l'amener à la FS la plus proche.

Après avoir administré les questionnaires, l'enquêteur fournira la réponse correcte aux questions portant sur les connaissances en matière de fécondité, de nutrition et autres thèmes, de sorte que l'enquête constitue un outil d'enseignement aussi bien que de collecte des données. Les enquêteurs seront formés pendant deux ou trois jours supplémentaires dans ce sens.

Dans l'aire sanitaire de Bassemyam dans la zone B, un ADBC (une femme) avait perdu au cours de la même année deux de ses enfants âgés respectivement de 7 et 12 ans. Passé la période douloureuse qui a suivi ces événements, elle reprend son sac d'ADBC et entreprend de poursuivre ses activités de sensibilisation à travers les VAD et les causeries-débats. Lorsqu'elle abordait le sujet de la PF certains lui répliquaient en ces termes: «C'est bien beau la PF, mais dis-nous: que serais-tu devenue si tu n'avais eu que 2 enfants? », faisant allusion à aux deux enfants décédés. Évidemment la causerie tournait court en ce moment.

Cette anecdote appelle deux réflexions: 1. La PF en tant que moyen d'espacement des naissances n'est pas très bien assimilée au sein des communautés rurales, elle est le plus souvent associée à «arrêt de la procréation ». Cette confusion semble provenir des terminologies en Moore: «yaag rogm » (espacer les naissances) et « guidg rogm» (empêcher les naissances). L’ADBC doit donc maîtriser ces tournures pour arriver à convaincre ses interlocuteurs.

2. La mortalité, surtout infantile reste élevée dans les zones rurales. De ce fait, il n'est pas aisé de faire passer un message de PF auprès d'une population qui connaît chaque jour dans son entourage un cas de décès d'enfants.

L'expérience des deux enquêtes (enquête de base de 1996, enquête de passage en 1998) nous amène à poser un certain nombre de problèmes que l'on peut appeler «éthique des enquêtes de PF en milieu rural ». La collecte des données dans les enquêtes en milieu rural, notamment tout ce qui touche à la 
sexualité et à la PF demeurent délicates tant pour le personnel de terrain que pour les $\mathrm{ADBC}$, vu la sensibilité des sujets d'une part et d'autre part compte tenu de certains facteurs conjoncturels. Les séances de formation des enquêteurs ne permettent pas d'anticiper toutes les situations car les réalités du terrain sont parfois dramatiques.

En 1998 l'enquête de passage se déroulait dans une situation de disette ce qui compliquait les entretiens. Nous avons très souvent entendu dire: "vous connaissez nos vrais problèmes, ceux qui n'ont pas besoin de questionnaire pour être élucidés: allez-y dire à Ouagadougou que nous avons faim! ». Ou encore pour ceux qui ne posent pas le problème à l'introduction le feront avec humour dans la section «PF et préférences en matière de fécondité » en ces termes: «vous savez bien qu'il n'y a pas d'érection quand le ventre est vide; alors comment voulez-vous que j'utilise le préservatif par exemple, puisqu'il semble qu'il faut le porter au moment de l'érection! »

L'enquête de base s'est déroulée pendant une épidémie de méningite (avril 1996). Dans un des villages de la zone $\mathrm{C}$, nous fûmes confrontés à des situations où dans certaines concessions des femmes avaient perdu parfois jusqu'à deux enfants. Est-il possible dans ces conditions de lui demander si elle «compte utiliser une méthode de PF dans les 12 prochains mois » alors que rien n'était fait par ailleurs pour sauver ce qui restait de sa progéniture? Peut-on expliquer à ces populations pourquoi on serait prêt à leur offrir des contraceptifs gratuitement ${ }^{15}$ alors que la FS n'est pas en mesure d'offrir le moindre comprimé de chloroquine?

\footnotetext{
${ }^{15}$ Une des questions de la section PF du questionnaire était «Si les produits contraceptifs vous étaient offerts gratuitement, accepteriez-vous de les utiliser? »
} 


\section{REFERENCES}

1. BONGAARTS J. (1991), "The KAP-gap and the unmet need for contraception", Population and Development Review, 17, 2, 293-313.

2. BONGAARTS J. (1990), "The measurement of unwanted fertility", Population and Development Review, 16, 3, 487-506.

3. DHS (1993). "Demographic and Health Survey - Burkina Faso". Calverton, Maryland USA: Institut National de la Statistique et de la Démographie \& Demographic and Health Surveys Macro International Inc.

4. PHILLIPS J.F., BINKA F.N., ADJUIK M. et al. (1997), "The determinants of contraceptive innovation: A case-control study of family planning acceptance in a traditional African society", Population Council Working Papers, 93, 38.

5. PRITCHETT L.H. (1994), "Desired fertility and the impact of population policies", Population and Development Review, 20, 1, 1-55.

6. UERD (1997). "Laboratoire de santé communautaire (LSC) - Rapport provisoire de l'enquête quantitative de base". Ouagadougou (unpublished). 